\title{
Human Pluripotent Stem Cell-Derived Neurons Are Functionally Mature In Vitro and Integrate into the Mouse Striatum Following Transplantation
}

\author{
Andrea Comella-Bolla ${ }^{1,2,3,4,5}$ (D) Javier G. Orlandi ${ }^{6,7,8}$ (D) Andrés Miguez $^{1,2,3,4,5}$ (D) Marco Straccia ${ }^{1,2,3,4,5}$ (D) \\ María García-Bravo $^{9,10}$ (D) - Georgina Bombau ${ }^{1,2,3,4,5}$ • Mireia Galofré ${ }^{1,2,3,4,5}$ (D) Phil Sanders ${ }^{1,2,3,4,5}$ (D) \\ Jordi Carrere $^{1,2,3,4,5}$ (D) José Carlos Segovia ${ }^{9,10}$ (D) - Joan Blasi ${ }^{11}$ (D) - Nicholas D. Allen ${ }^{12} \cdot$ Jordi Alberch $^{2,3,4,5,6}$ (D) \\ Jordi Soriano $^{7,13}$ (D) Josep M. Canals ${ }^{1,2,3,4,5}$ (D)
}

Received: 13 July 2019 / Accepted: 23 March 2020 / Published online: 30 April 2020

(C) The Author(s) 2020

\begin{abstract}
Human pluripotent stem cells (hPSCs) are a powerful tool for modelling human development. In recent years, hPSCs have become central in cell-based therapies for neurodegenerative diseases given their potential to replace affected neurons. However, directing hPSCs into specific neuronal types is complex and requires an accurate protocol that mimics endogenous neuronal development. Here we describe step-by-step a fast feeder-free neuronal differentiation protocol to direct hPSCs to mature forebrain neurons in 37 days in vitro (DIV). The protocol is based upon a combination of specific morphogens, trophic and growth factors, ions, neurotransmitters and extracellular matrix elements. A human-induced PSC line (Ctr-Q33) and a human embryonic stem cell line (GEN-Q18) were used to reinforce the potential of the protocol. Neuronal activity was analysed by single-cell calcium imaging. At 8 DIV, we obtained a homogeneous population of hPSC-derived neuroectodermal progenitors which self-arranged in bi-dimensional neural tube-like structures. At 16 DIV, we generated hPSC-derived neural progenitor cells (NPCs) with mostly a subpallial identity along with a subpopulation of pallial NPCs. Terminal in vitro neuronal differentiation was confirmed by the expression of microtubule associated protein 2b (Map 2b) by almost 100\% of hPSC-derived neurons and the expression of specific-striatal neuronal markers including GABA, CTIP2 and DARPP-32. HPSC-derived neurons showed mature and functional phenotypes as they expressed synaptic markers, voltage-gated ion channels and neurotransmitter receptors. Neurons displayed diverse spontaneous activity patterns that were classified into three major groups, namely "high",
\end{abstract}

Electronic supplementary material The online version of this article (https://doi.org/10.1007/s12035-020-01907-4) contains supplementary material, which is available to authorized users.

\author{
Phil Sanders \\ phil.sanders@ub.edu \\ Jordi Carrere \\ jambiol@hotmail.com \\ José Carlos Segovia \\ jc.segovia@ciemat.es \\ Joan Blasi \\ blasi@ub.edu \\ Nicholas D. Allen \\ AllenND@ cardiff.ac.uk \\ Jordi Alberch \\ alberch@ub.edu \\ Jordi Soriano \\ jordi.soriano@ub.edu
}

Mireia Galofré

mireiagalofre@ub.edu

Extended author information available on the last page of the article 
"intermediate" and "low" firing neurons. Finally, transplantation experiments showed that the NPCs survived and differentiated within mouse striatum for at least 3 months. NPCs integrated host environmental cues and differentiated into striatal mediumsized spiny neurons (MSNs), which successfully integrated into the endogenous circuitry without teratoma formation. Altogether, these findings demonstrate the potential of this robust human neuronal differentiation protocol, which will bring new opportunities for the study of human neurodevelopment and neurodegeneration, and will open new avenues in cell-based therapies, pharmacological studies and alternative in vitro toxicology.

Keywords Telencephalon $\cdot$ Neuronal differentiation $\cdot$ Striatum $\cdot$ Calcium imaging $\cdot$ Spike-inference analysis $\cdot$ Transplantation

\section{Introduction}

Neurogenesis is a complex and highly regulated process directed by evolutionary conserved signalling molecules [1]. Despite intensive research, many aspects of neurogenesis and neurodevelopment remain to be elucidated. Furthermore, recent evidence indicates that neurodegenerative disorders such as Huntington's disease (HD) display alterations early during neurogenesis that may contribute to neurodegeneration later in life [2-4]. Thus, methods to facilitate the understanding of neurogenesis and the contribution of neurodevelopmental alterations to neurodegenerative diseases are required.

In recent years, human embryonic stem cells (hESCs) and human-induced pluripotent stem cells (hiPSCs), collectively known as human pluripotent stem cells (hPSCs), have emerged as versatile and powerful tools for research and applied medicine given their ability to differentiate into any cell type of the human body including neurons [5]. Thus, hPSCs provide a powerful tool to gain insight into the mechanisms underlying human neurogenesis and enhance understanding of how neurodevelopment is altered in neurodegenerative diseases. Furthermore, hPSC-based therapies for the treatment of neurodegenerative diseases are possible by the differentiation of hPSCs to the affected neuronal type for subsequent transplantation into patients.

Differentiating hPSCs to a specific neuronal type is a complex endeavour that requires a robust effective in vitro differentiation protocol that mimics in vivo neurodevelopment through the addition of the necessary morphogens, growth factors, small molecules and other components at the relevant concentration and time [6-8]. In vitro neuronal differentiation can be broadly divided into three main phases, namely neural induction, neural progenitor patterning and terminal neuronal differentiation and maturation.

Neural induction of hPSCs requires the inhibition of Wnt and bone morphogenetic protein (BMP) signalling which would otherwise drive the hPSCs to a non-neural fate [9]. Indeed, it was observed that the absence of BMP signalling induces the expression of neural-specific factors [10]. Inhibition of the transforming growth factor beta (TGF $\beta$ ) and canonical Wnt pathways are also required for neural induction as these pathways are implicated in maintaining pluripotency $[11,12]$.
During neurodevelopment, neural progenitor cells (NPCs) are patterned along the dorsoventral (DV) and anteroposterior (AP) axes of the neural tube to generate the progenitors that will produce the numerous different neuronal types of the forebrain, midbrain and hindbrain [1]. Following specification of a forebrain identity, gradients of TGF $\beta$ and BMP signalling specify a dorsal NPC identity which gives rise to cortical neurons [13]. Conversely, Sonic hedgehog (SHH) signalling induces a ventral identity which produces the ganglionic eminence NPCs that give rise to striatal neurons among others $[14,15]$.

Following the differentiation of the patterned NPCs to neurons, terminal neuronal differentiation and maturation are required for neuronal survival, plasticity and brain circuit integrity and function. For neurons to correctly differentiate and mature, they must progressively gain excitability, and acquire passive and active neuronal properties able to generate and transmit action potential [16]. Neuronal maturation depends on the synergistic action of developmental transcription factors (TFs), the activation or inhibition of intracellular signalling pathways, cell-to-cell interactions, and extracellular factors such as neurotransmitters (NTs), neurotrophic factors or ionic concentrations [17].

The generation of functional mature neurons differentiated from hPSCs is essential for the study of neuronal differentiation and also neuronal activity at both the individual neuron and network level. We have previously reported that the application of a cocktail of soluble molecules to hPSC-derived NPCs generates electrophysiologically active neurons that display relative negative membrane potentials and elicit spontaneous trains of action potentials [18].

A successful neuronal differentiation protocol must be robust and generate a similar outcome with different hPSC lines. Ideally, a neuronal differentiation protocol should generate functionally mature neurons in a relatively short amount of time to accelerate investigation. Furthermore, the differentiation of the cells as they progress through the protocol must be well-characterised at the gene expression level to demonstrate that the protocol mirrors the gene expression dynamics that occur in vivo. Also, the neurons that are generated should be characterised at the functional level to demonstrate that their activity is comparable with that of the equivalent in vivo neurons. Finally, in addition to in vitro analyses, from a cell therapy perspective, it must be possible to transplant the hPSC- 
derived NPCs into the relevant brain area where they should survive, differentiate into neurons and integrate into the host brain circuitry.

In this work, we provide a step-by-step characterisation at the gene expression and functional levels of the differentiation of hiPSC and hESC lines to functionally mature ventral forebrain neurons, including striatal medium spiny neurons, in a relatively short period of time. We observed the production of patterned NPCs after 16 days in vitro (DIV) which subsequently differentiated to spontaneously active and synaptically mature neurons in a further 21 days (37 DIV). Gene expression analysis using our highthroughput quantitative PCR (qPCR) platform to assess forebrain neuronal differentiation revealed gene expression dynamics similar to those observed during neurodevelopment [19]. Spontaneous neuronal activity was demonstrated using a single-cell calcium $\left(\mathrm{Ca}^{2+}\right)$ imaging assay in combination with a novel semi-automatic MATLAB-based software toolbox (NETCAL) that we developed previously [20]. We also transplanted hPSCderived NPCs into neonatal mouse striatum where they differentiated to neurons, survived for a prolonged period, projected axons to the relevant host brain target areas and established synaptic connections.

Overall, we present a fast and robust method for the differentiation of hPSC to neurons which will help advance the study of human neurodevelopment, the treatment of neuronal disorders, and will facilitate the design of high-throughput drug screening and in vitro neurodevelopmental toxicology procedures and stem cell-based therapies.

\section{Material and Methods}

\section{Animals}

B6CBA mice were obtained from Jackson Laboratory (Bar Harbor, ME, USA). The animals were housed with access to food and water ad libitum in a colony room kept at $19-22{ }^{\circ} \mathrm{C}$ and 40-60\% humidity, under a 12:12-h light/dark cycle. Experiments were carried out according to the European regulation (2010/63/UE) for the care and use of laboratory animals.

\section{Human Stem Cell Lines}

We compared the control (Ctr) Ctr-Q33, a hiPSC line, with 33 CAG repeats (generous gift from C. N. Svendsen, Cedar Sinai, Los Angeles, CA; USA: CS83iCTR-33nxx) and GENEA (GEN)-Q18, an ESC line, with 15/18 CAGs (GENEA019; derived from GENEA Biocells; Australia; hPSCreg Name: GENEAe020-A). All cells were regularly tested for mycoplasma contamination and karyotype normality. Human cells are growth in a $37{ }^{\circ} \mathrm{C}$ incubator with a humidified atmosphere and 5\% CO2. G-band karyotypes were made in an external centre, Ambar-Anàlisis Mèdicas (Barcelona, Spain).

\section{GEN-Q18 hESC Expansion}

GEN-Q18 hESC was cultured in feeder-free matrigel coated 6-well plates in $2.5 \mathrm{ml}$ of Genea M2 Medium (MED-02$250 \mathrm{ml}$; 17G13CCMO23; Genea Biocells), a serum-free fully-defined medium containing bovine serum albumin (BSA), bFGF, Activin A and growth factors. Medium was changed every 2 days and daily in the 2 days before passaging due to the higher cell confluency.

After a minimum of 4 passages in Genea M2 medium, GEN-Q18 hESC was adapted and expanded in MTESR1 medium as we describe below.

\section{Human PSC Maintenance in mTeSR1 Media}

All hPSCs were cultured on matrigel (354230; Corning Inc., NY, USA) feeder-free coated 6-well plates in $2 \mathrm{ml}$ of serumfree defined mTeSR1 medium which contains recombinant human $\mathrm{bFGF}$ and recombinant human TGF $\beta$ (complete mTeSR1: mTeSR1 basal medium (05850) and mTeSR1 supplemented 5x (05852); StemCell Technologies Inc., MA, USA). Medium was daily changes (every $24 \mathrm{~h} \pm 2$ ).

\section{Human Pluripotent Stem Cells Passaging Protocol with ROCK Inhibitor}

HPSC cultures were passaged when cell confluence reached 70-80\% (twice per week). Cells were gently washed twice with $2 \mathrm{ml}$ of Dulbecco's (D)-phosphate-buffered saline (PBS) without calcium and magnesium (D-PBS ${ }^{-/}, 14,190$ 250; Thermo Fisher Scientific Inc., MA, USA) and incubated for 20-25 min with dispase in DMEM/F12 (07923; Stem cell Technologies, Cambridge, UK) with $10 \mu \mathrm{M}$ of ROCK inhibitor (Y-27632; EVOTEC Ltd. Milton, UK) at $37^{\circ} \mathrm{C}$. Then, dispase was removed and cells were harvested in DMEM/F12 (Stem cell Technologies) media by pipetting up and down using a 5 -ml pipette to avoid single-cell desegregation. Cells were centrifuged at $1000 \mathrm{rpm}$ for $3 \mathrm{~min}$ and resuspended with warm and fresh Complete-mTeSR 1 medium with $10 \mu \mathrm{m}$ of ROCK inhibitor (Abcam Inc.) at the desired dilution. Then, cell suspension was seeded on tempered matrigel coated 6-well plates. Differentiated colonies were removed manually using a stereotypic microscope and a $10-\mathrm{ml}$ sterile tip prior passaging.

\section{Human PSC Neuronal Differentiation Protocol}

A schematic illustration of the differentiation protocol is shown in Fig. 1. At 60-70\% of cell confluence, hPSC cultures 
Fig. 1 Sketch of the protocol for the in vitro differentiation of hPSCs into mature forebrain neurons in 37 DIV. The first phase consists of the neural induction of pluripotent stem cell colonies with dual-SMAD and Wnt inhibition. During the second phase, NE progenitors are regionalized to ventral telencephalic neural progenitors by inhibition of both dorsal Wnt and BMP morphogens. Finally, during the third phase, neuronal differentiation and maturation are promoted by applying a cocktail of small molecules that stimulate cell cycle exit and neuronal maturation

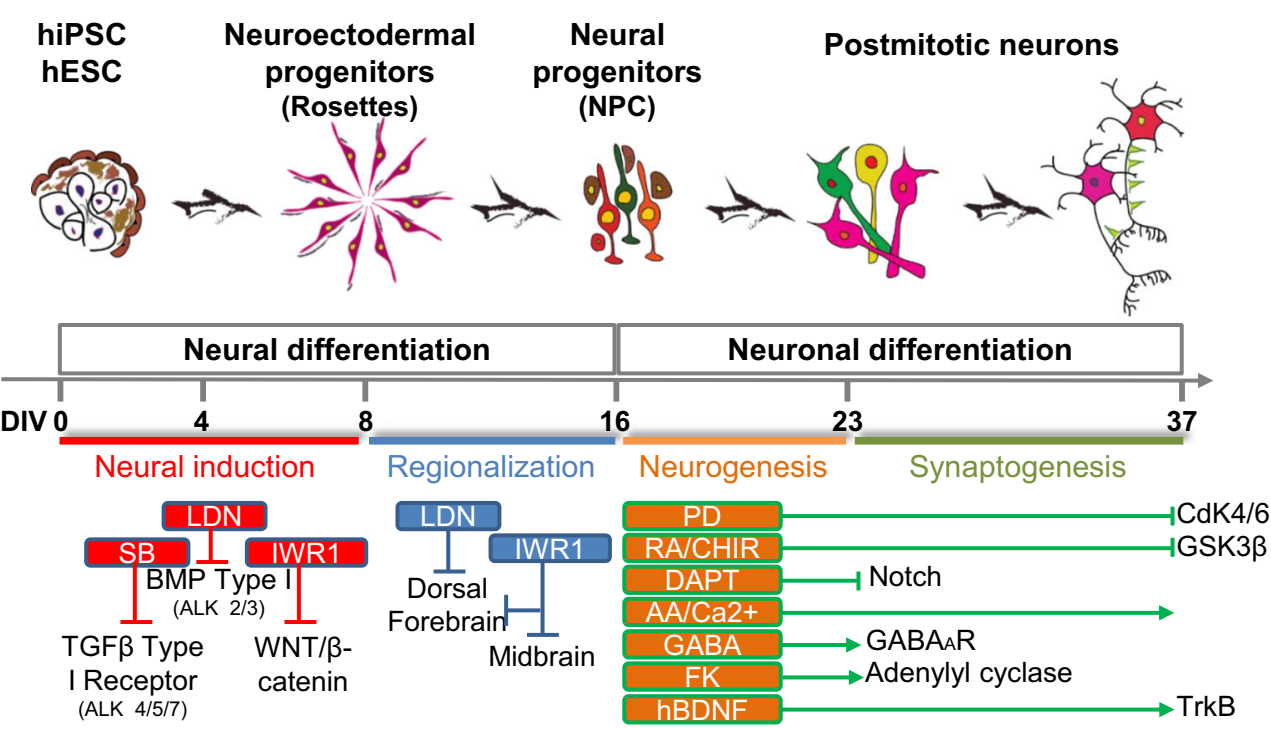

were washed 3 times with $2 \mathrm{ml}$ of D-PBS with calcium and magnesium $\left(\mathrm{PBS}^{+/+}\right.$, 14040-091; Thermo Fisher Scientific Inc., MA, USA). The medium was then replaced by neural induction SLI medium from 0 DIV until 8 DIV (SLI culture media: Advanced DMEM/F-12(1X) (12634-010; Thermo Fisher Scientific Inc.) with $2 \mathrm{mM}$ of GlutaMax ${ }^{\mathrm{TM}} 100 \mathrm{x}$ (35050-038, Thermo Fisher Scientific Inc.), 1\% penicillin/ streptomycin (P/S; 15140122; Thermo Fisher Scientific Inc., MA, USA); $10 \mu \mathrm{M}$ SB 431542 (SB; 447536; EVOTEC Ltd. Milton, UK.); 1 MM LDN 193189 (396388; EVOTEC Ltd. Milton, UK); $1.5 \mu \mathrm{M}$ IWR1 (476979; EVOTEC Ltd. Milton, UK), and 2\% B27-supplemented without RA (50X) (12587010; Thermo Fisher Scientific Inc.)).

At $4 \mathrm{DIV}$, confluent cultures were treated with $10 \mu \mathrm{M}$ of ROCK inhibitor (Abcam Inc.) for $1 \mathrm{~h}$, washed twice with $2 \mathrm{ml}$ of PBS without calcium and magnesium (Thermo Fisher Scientific Inc.), incubated with accutase (StemPro A11105; Thermo Fisher Scientific Inc.) for $7 \mathrm{~min}$ for cell dissociation and passaged onto matrigel-coated plates, with a split ratio of 1:2. On 8 DIV, cultures were passaged 1:2 and cultured in LI medium until 15 DIV (LI culture media: Advanced DMEM/F12(1X) (Thermo Fisher Scientific Inc.) with $2 \mathrm{mM}$ of GlutaMax TM 100x (Thermo Fisher Scientific Inc.), 1\% P/S (Thermo Fisher Scientific Inc.), 200 nM LDN 193189, $1.5 \mu \mathrm{M}$ IWR 1 and 2\% B27-supplemented without RA (50X) (Thermo Fisher Scientific Inc.)).

Cultures received daily medium changes of $2 \mathrm{ml}$ except from DIV 5 to DIV 7 (both included), when $4 \mathrm{ml}$ of fresh medium were added. At 16 DIV, NPCs were washed 3 times in $\mathrm{PBS}^{-/}$(Thermo Fisher Scientific Inc.), dissociated with accutase for $5 \mathrm{~min}$ and plated with a population of $8 \times 10^{4}$ cells in 12-mm-diameter glass coverslips (treated and coated). Prior to use, coverslips were cleaned with $70 \%$ nitric acid, washed 5 times with deionized water and one time with absolute ethanol (1009832500; CAS 64-17-5; MERCK Millipore,
Darmstadt, Germany) and oven sterilized at $180{ }^{\circ} \mathrm{C}$ o.n. CSs were coated first with $100 \mu \mathrm{g} / \mathrm{ml}$ poly-D-lysine (P0899$100 \mathrm{mg}$; Sigma-Aldrich) in borate buffer (15663; FlukaSigma-Aldrich Chemie GmbH; Steinheim, Germany) and with matrigel. Cells were differentiated for additional 21 DIVs in SCM1/2 media (SCM1 from 16 DIV until 23 DIV and SCM2 from 23 DIV until 37 DIV changed every 2-3 days) (SCM1 culture media (changed at 16 DIV, 18 DIV and 21 DIV): Advanced DMEM/F-12 (1X) (Thermo Fisher Scientific Inc.) with $2 \mathrm{mM}$ of GlutaMax ${ }^{\mathrm{TM}}$ 100x (Thermo Fisher Scientific Inc.); $1 \%$ P/S (Thermo Fisher Scientific Inc.); 2\% B27-supplemented (50X) (17504-044; Thermo Fisher Scientific Inc.); 2 $\mu \mathrm{M}$ PD 0332991 (482855; EVOTEC Ltd. Milton, UK); $10 \mu \mathrm{M}$ DAPT (396736; EVOTEC Ltd. Milton, UK); $0.6 \mathrm{mM} \mathrm{CaCl2} \mathrm{(to} \mathrm{give}$ $1.8 \mathrm{mM}$ final complete medium; MERCK, CAT, Spain), $200 \mu \mathrm{M}$ ascorbic acid (AA; A4544-100G; Sigma-Aldrich, Madrid, Spain), 10 ng/ml hBDNF (450-02; Peprotech Inc., NJ, USA); $10 \mu \mathrm{M}$ Forskolin (FK,000087; EVOTEC Ltd.), 3 MM CHIR 99021 (CH; 401952; EVOTEC Ltd. Milton, UK) and $300 \mu \mathrm{M}$ GABA (0.44; Tocris Cookson Inc., MO, USA)). (SCM2 culture media: (1:1) of Advanced DMEM/F12 (1X) (Thermo Fisher Scientific Inc.) with $2 \mathrm{mM}$ of GlutaMax $^{\text {TM }}$ 100x (Thermo Fisher Scientific Inc.): Neurobasal A (10888-022; Thermo Fisher Scientific Inc.); $1 \% \mathrm{P} / \mathrm{S}$ (Thermo Fisher Scientific Inc.), 2\% B27supplemented (50X) (Thermo Fisher Scientific Inc.); $2 \mu \mathrm{M}$ PD (EVOTEC Ltd.); $3 \mu \mathrm{M}$ CH (EVOTEC Ltd.); $0.3 \mathrm{mM}$ $\mathrm{CaCl} 2$ (to give $1.8 \mathrm{mM}$ final complete medium MERCK); $200 \mu \mathrm{M}$ AA (Sigma) and $10 \mathrm{ng} / \mathrm{ml}$ hBDNF (Peprotech Inc.)).

\section{NPC Transplantation in Neonatal Mouse Striatum}

To track the cells after transplants, Ctr-Q33 hiPSC line was transduced with green fluorescent protein (GFP) lentivirus under the 
control of the constitutive elongation factor alpha $(E F 1-\alpha)$ promoter during WiCell maintenance. Lentivirus was incubated for $2 \mathrm{~h}$ at $37^{\circ} \mathrm{C}$. Cultures were grown and maintained in WiCell procedure until cell sorting by FACS (fluorescence-activated cell sorting). After three passages, transfected cells were sorted by the expression of GFP. Sorted cells were grown and maintained in WiCell procedure and adapted and expanded to mTesR1 medium after karyotyping test.

Ctr3-Q33-GFP NPCs at 16 DIV were harvested and resuspended in D-PBS ${ }^{-/}$(Thermo Fisher Scientific Inc.). P (postnatal day) 2 neonatal mice were anaesthetised by hypothermia and placed on ice until cessation of movement. Unilateral striatal injections were performed using a stereotaxic apparatus (Davis Kopf Instruments, Tujunga, CA, USA) and a 10- $\mu$ l Hamilton syringe with a 33-gauge needle (Hamilton, Reno, $\mathrm{NV}$ ), setting the following coordinates (millimetres): AP, + $2.3, \mathrm{~L},+1.4$ from lambda and dorsoventral, -1.8 from dura. Every animal received 15,000 cells diluted in $1 \mu \mathrm{l}$ of D-PBS ${ }^{-1}$ (Thermo Fisher Scientific Inc.). Upon completion of stereotaxic surgery, pups were warmed, monitored for $1 \mathrm{~h}$ to ensure recovery and then returned to the housing facility.

\section{Immunocytochemistry}

HPSC-derived cultures were fixed for $20 \mathrm{~min}$ at room temperature (r.t.) with $200 \mu \mathrm{l} / 24$-well of $4 \%$ paraformaldehyde solution (P/0840/53; Fisher Scientific UK Limited, Leicestershire, UK) and washed three times with 1x PBS and stored at $4{ }^{\circ} \mathrm{C}$ in $500 \mu \mathrm{l} / 24$-well with $0.03 \%$ sodiumazide (71289; Fluka-Sigma-Aldrich) until use. For immunolabelling, samples were blocked and permeabilized for 45 min at r.t. with PTB solution (PBS 1x with $0.3 \%$ Triton X-100 (T8532; Sigma-Aldrich Quimica SL., Madrid, Spain), $0.03 \%$ sodium-azide, 1\% BSA (A9647-1006; SigmaAldrich, Madrid, Spain) and 5\% normal goat serum (NGS; S-1000; Vector Laboratories Ltd., UK) and/or 5\% donkey serum (NDS; 017-000-121; Jackson Immuno Research Laboratories Inc.; PA, USA)), before being incubated overnight (o.n.) at $4{ }^{\circ} \mathrm{C}$ with the appropriate primary antibodies (Antibody references and dilutions are defined in Table 1). After o.n. incubation, primary antibodies were removed, and samples were washed 3 times for 10 min with PBS 1x upon the tilt table. Then, cells were incubated for $1-2 \mathrm{~h}$ at RT in darkness with fluorophore-conjugated secondary antibodies (References and dilutions are detailed in Table 1). After three washes of 10 min with PBS 1x, cells were counterstained with nuclear staining with DAPI $300 \mathrm{nM}$ (D1306; Thermo Fisher Scientific). Coverslips were mounted in Fluoromount-G media (0100-01; Southern Biotech, AL, USA) and imaged using an Olympus IX71 inverted microscope equipped with an Orca Flash 4.0 camera (C11440-22CU, Hamamatsu Photonics France sucursal España, Barcelona, Spain) or the Leica SP5
TCS two-photon laser scanning confocal microscope (Leica Microsystems, Wetzlar, Germany).

\section{Immunohistochemistry}

Animals were deeply anaesthetised with pentobarbital and intracardially perfused with PBS $1 \mathrm{x}$ and a $4 \%$ paraformaldehyde solution $(\mathrm{P} / 0840 / 53$; Fisher Scientific UK Limited, Leicestershire, UK) in $0.1 \mathrm{M}$ sodium phosphate (CAS 7601-54-9; Sigma-Aldrich, Madrid, Spain). Brains were removed and post-fixed o.n. in the same solution, washed three times with PBS $1 \mathrm{x}$, cryoprotected with $30 \%$ sucrose (CAS 57-50-1; Sigma-Aldrich, Madrid, Spain) in PBS $1 \mathrm{x}$ and frozen in dry-ice cooled methylbutane (CAS 78-78-4; Sigma-Aldrich, Madrid, Spain). Serial coronal sections $(20 \mu \mathrm{m})$ of the brain were obtained using a cryostat (Microm $560 \mathrm{M}$, Thermo Fisher). Tissue was first incubated with a blocking solution containing PBS $1 \mathrm{x}, 0.3 \%$ Triton X-100 (Sigma-Aldrich Quimica SL.) and 5\% normal horse serum (31874; Thermo Scientific, IL, USA) for $2 \mathrm{~h}$ at RT. Brain sections were then incubated o.n. at $4{ }^{\circ} \mathrm{C}$ with different primary antibodies diluted in the blocking solution (see Table 1). After three washes with PBS $1 \mathrm{x}$, tissue was incubated for $1 \mathrm{~h}$ and a half at RT with specific fluorophore-conjugated secondary antibodies. Images were acquired with a Leica SP5 TCS twophoton laser scanning confocal microscope (Leica Microsystems).

\section{Immunogold Labelling and Transmission Electron Microscopy}

For transmission electron microscopy (TEM) studies, samples were fixed with a solution of $2 \%$ PFA $/ 0.5 \%$ glutaraldehyde in $0.1-\mathrm{M}$ phosphate buffer, post-fixed with $1 \%$ osmium tetroxide, dehydrated and embedded in epoxy resin. Semi-thin sections $(1 \mu \mathrm{m})$ were stained with methylene blue. Ultra-thin sections $(70 \mathrm{~nm})$ were immunolabelled with primary antibody, followed by incubation with a secondary antibody conjugated with $10 \mathrm{~nm}$ electron-dense colloidal gold (Aurion, Electron Microscopy Sciences). GFP antibody (Abcam) was used for detecting human cells. Images were acquired with a JEOL 1010 transmission electron microscope equipped with a CCD Orius camera (Gatan).

\section{Unbiased Cell Counts}

\section{Neural Progenitor Cell Counts by CellProfiler Software}

HPSC-derived NPC populations at 16 DIV were quantified using an open-access CellProfiler software (BROAD institute, 
Table 1 List of primary and secondary antibodies used in the work

\begin{tabular}{|c|c|c|c|c|c|}
\hline \multicolumn{6}{|l|}{ Primary antibodies } \\
\hline Target & Supplier & Reference & Host & Dilution & Used \\
\hline $5-\mathrm{HT}$ & Sigma & S5545 & $\mathrm{R} *$ & $1 / 5000$ & $\mathrm{ICC} * *$ \\
\hline ChAT & Chemicon & $\mathrm{AB} 144 \mathrm{P}$ & $\mathrm{G}^{*}$ & $1 / 100$ & ICC \\
\hline CTIP2 (25B6) & Abcam & ab18465 & Rat & $1 / 300$ & $\mathrm{ICC}$ \\
\hline DARPP-32 (H62) & Santa Cruz Biotechnology & Sc-11365 & $\mathrm{R}$ & $1 / 100$ & ICC \\
\hline DARPP-32 (19A3) & Cell signalling & 2306 & $\mathrm{R}$ & $1 / 500$ & $\mathrm{IHC} * *$ \\
\hline DLX_pan & CHD1 & PA5967 & $\mathrm{R}$ & $1 / 150$ & ICC \\
\hline $\mathrm{EBF}_{-}$pan & CHD1 & PA590 & $\mathrm{R}$ & $1 / 200$ & ICC \\
\hline GABA & Sigma & A2052 & $\mathrm{R}$ & $1 / 500$ & $\mathrm{ICC}$ \\
\hline GFP & Abcam & Ab6556 & $\mathrm{R}$ & $1 / 500$ & TEM \\
\hline Human Nuclei (HNA) & Millipore & MAB4383 & $\mathrm{M} *$ & $1 / 100$ & $\mathrm{IHC}$ \\
\hline MAP2b & BD Transduction Laboratories & 610460 & M & $1 / 500$ & ICC \\
\hline MAP2 $(2 a+2 b)(A P-20)$ & Sigma & M1406 & M & $1 / 500$ & $\mathrm{IHC}$ \\
\hline Nestin & Millipore & MAB5326 & M & $1 / 200$ & ICC \\
\hline OCT3-4 (C-10) & Santa Cruz Biotechnology & sc-5279 & M & $1 / 100$ & ICC \\
\hline PAX6 & Hybrido Bank & Pax6 & M & $1 / 200$ & ICC \\
\hline PLZO (2A9) & Calbiochem & OP128 & M & $1 / 100$ & ICC \\
\hline PSD-95 & Abcam & $\mathrm{Ab} 41455$ & $\mathrm{R}$ & $1 / 150$ & $\mathrm{ICC}$ \\
\hline SNAP25 & Abcam & Ab41455 & $\mathrm{R}$ & $1 / 150$ & ICC \\
\hline Synaptophysin & Synaptic Systems & 101011 & M & $1 / 1000$ & $\mathrm{ICC}$ \\
\hline TBR1 & Abcam & Ab31940 & $\mathrm{R}$ & $1 / 500$ & $\mathrm{ICC}$ \\
\hline $\mathrm{TH}$ & Abcam & $\mathrm{AB} 152$ & $\mathrm{R}$ & $1 / 300$ & ICC \\
\hline ZO-1 & Invitrogen & $40-2200$ & $\mathrm{R}$ & $1 / 100$ & ICC \\
\hline B-III tubulin & Sigma & $\mathrm{T} 220$ & $\mathrm{R}$ & $1 / 500$ & ICC \\
\hline SNAP25 (SMI 81) & BioLegend & 836306 & M & $1 / 1000$ & WB $* *$ \\
\hline Syntaxin (HPC-1) & Gift of Dr. C. Barnstable & & M & $1 / 2000$ & WB \\
\hline Synaptotagmin (41.1) & Synaptic Systems & 105011 & M & $1 / 1000$ & WB \\
\hline Actin (C4) & MP Biomedicals & 0869100 & M & $1 / 20000$ & WB \\
\hline \multicolumn{6}{|c|}{ Fluorophore-conjugated secondary antibodies } \\
\hline Target & Supplier & Reference & Host & Dilution & Used \\
\hline AF488 donkey $\alpha-\mathrm{M}$ & Jackson Immuno Research (JIR) & $715-545-150$ & $\mathrm{D} *$ & $1 / 500$ & ICC \\
\hline AF488 donkey $\alpha-\mathrm{R}$ & JIR & $711-545-152$ & $\mathrm{D}$ & $1 / 500$ & ICC \\
\hline Cy2 donkey $\alpha-\mathrm{G}$ & JIR & $705-225-003$ & $\mathrm{D}$ & $1 / 100$ & ICC \\
\hline Cy3 donkey $\alpha-\mathrm{R}$ & JIR & $711-165-152$ & $\mathrm{D}$ & $1 / 500$ & ICC \\
\hline Су $3 \alpha$-rat & JIR & $712-165-150$ & Rat & $1 / 500$ & ICC \\
\hline Phalloidin-TRITC & Sigma & P1951 & - & $1 / 500$ & ICC \\
\hline GFP-FITC & Abcam & Ab6662 & G & $1 / 200$ & $\mathrm{IHC}$ \\
\hline \multicolumn{6}{|c|}{ HRP-conjugated secondary antibodies } \\
\hline $\mathrm{HRP} \alpha-\mathrm{M}$ & Dako & P0161 & & $1 / 5000$ & WB \\
\hline
\end{tabular}

*Host: $M$ mouse; $R$ rabbit; $G$ goat; $D$ donkey

**Used: ICC immunocytochemistry; IHC immunohistochemistry; WB western blot
MA, USA). Nineteen random images, corresponding to $3 \%$ of a 24-well plate $\left(1.92 \mathrm{~cm}^{2}\right)$ culture, were taken with the epifluorescence Leica AF600 microscope (Leica Microsystems). Images were loaded in a customized pipeline with an initial nuclei detection by DAPI immunofluorescence and then the second channel, green, and the third channel, red, immunolabelled detected-nuclei counts.

\section{Neuronal Cell-Type Counts by CAST Program}

HPSC-derived neuronal cell types at 23 DIV and 37 DIV were manually counted using a nonbiased computer-assisted stereological toolbox (CAST) program (Olympus America Inc., NY, USA). We counted the $3 \%$ of a 12 -mm-diameter coverslip $\left(1.2 \mathrm{~cm}^{2}\right)$ culture area. 


\section{Graft Size and Neuronal Counts of Transplanted Cells}

Graft size was calculated by delineating the outer perimeter of $\mathrm{GFP}^{+}$cells in eight transplanted mice. The volume occupied by the graft core was estimated through extrapolation of the area quantified in sections spaced $120 \mu \mathrm{m}$ apart, by using an Olympus optical microscope and CAST stereology software. For determining the neuronal fate of transplanted cells, immunofluorescence images were acquired with a TCS SP5 confocal microscope (Leica) and manually counted using the ImageJ and CAST software. Immunolabelled cells in the regions of interest were quantified using high intensity projection of $\mathrm{Z}$ stacked images on five evenly-spaced coronal sections from six transplanted mice at each time point.

\section{Calcium Imaging}

Sixteen DIV NPCs seeded upon 12-mm-diameter glass coverslips were differentiated into neurons and monitored for their spontaneous dynamics at 37 DIV through multi-neuron fluorescence calcium imaging. This technique enabled the simultaneous, large population tracking of neuronal firing by the binding of $\mathrm{Ca}^{2+}$ ions to a fluorescent indicator. Recordings were performed in artificial cerebrospinal fluid solution (aCSF) ( $\mathrm{pH}$ 7.4) composed by $128 \mathrm{mM} \mathrm{NaCl}$, $4 \mathrm{mM} \mathrm{KCl}, 1 \mathrm{mM} \mathrm{CaCl}_{2}, 1 \mathrm{mM} \mathrm{MgCl}, 45 \mathrm{mM}$ sucrose, $10 \mathrm{mM}$ glucose and $0.01 \mathrm{M}$ HEPES. Fluo4-acetoxymethyl ester (AM) (494/506 nm) (F14201; Thermo Fisher Scientific Inc.) fluorescent $\mathrm{Ca}^{2+}$ sensitive indicator was used in all measurements. Prior to imaging, cultures were placed in a 35-mmdiameter glass bottom chamber (P35G-0-14-C, MatTek Corporation, MA, USA) that contained $2 \mu \mathrm{M}$ of Fluo4-AM in a total volume of $2 \mathrm{ml}$ of aCSF, and gently incubated on an orbital shaker in darkness for $15 \mathrm{~min}$ at RT. Cultures were then washed twice with fresh aCSF and stabilized for $5 \mathrm{~min}$ at $37^{\circ} \mathrm{C}$ with $4 \mathrm{ml}$ of fresh aCSF. The recording chamber was mounted on a Zeiss inverted microscope equipped with an Orca Flash 2.8 CMOS camera (Hamamatsu Photonics) and a light source for fluorescence. Image sequences of neuronal activity were acquired for $10 \mathrm{~min}$ at RT, with a size of $960 \times$ 720 pixels, and a speed of 20 frames per second (FPS). Microscope settings combined a $\times 5$ objective with a $\times 0.63$ optical zoom, providing a spatial resolution of $4.40 \mu \mathrm{m} /$ pixel and a field of view (FOV) of $4.2 \times 3.2 \mathrm{~mm}^{2}$ that contained $3000-4000$ cells. Representative bright field and fluorescence images for a particular experiment are provided in Fig. S9.

\section{Neuronal Activity Analysis}

$\mathrm{Ca}^{2+}$ imaging recordings were analysed with NETCAL [20], a custom-built software package run in MATLAB. For each experiment, about 1000-3000 neurons (soma regions) were automatically selected on the FOV of the culture based on the brightness of the neuronal soma. Each soma denoted a region of interest (ROI) spanning a square area 7 pixels wide (about $645 \mu^{2}$ ). The fluorescence intensity of each ROI as a function of time, $\mathrm{F}^{*}(\mathrm{t})$, was obtained by averaging the colour intensity across all pixels within the ROI at every frame. To reduce noise, fluorescence traces were smoothed out by applying a "moving average" filter 5 frames $(250 \mathrm{~ms})$ wide. Traces were next corrected for small drifts by subtracting a smoothed spline of the original signal. The spline was constructed by diving the original signal in blocks $25 \mathrm{~s}$ wide, and by connecting in a spline manner the average value within each block. The drift correction effectively removed large time-scale fluctuations while preserving neuronal firing traits and the original baseline fluorescence level $\left(\mathrm{F}_{0}\right)$. Fluorescence signals were finally normalized as $F(t)=100\left(F^{*}-F_{0}\right) / F_{0} \equiv$ $\Delta F / F_{0}(\%)$. Examples of characteristic fluorescence traces are provided in Fig. S9.

Neurons were separated in two groups (active or inactive) based on their fluorescence profiles by means of supervised machine learning algorithms. Briefly, several features of the fluorescence traces were first extracted by identifying their intervals of high activity. These intervals were set as the time spanned by the signal above a given threshold. Next, about 100 representative traces for each group were manually selected, and the rest were automatically assigned to the corresponding group through a boosting algorithm [21]. Finally, for each active neuron, spike trains were inferred through the "peeling algorithm" $[22,23]$. For single-spike detection, we used a single-exponential model with a detection threshold of $0.8 \%$ $\Delta F / F_{0}$ and a decay time constant of tau $=1 \mathrm{~s}$.

To describe the activity properties of neurons, we defined a set of nine neuronal activity features detailed in Fig. S10. These features include number of spikes (NS); firing rate (FR, spikes along time, Hz); inter-spike interval (ISI, s); standard deviation (SD) of ISI (s); number of bursts (B; defined as concatenated spikes with intervals smaller than $1 \mathrm{~s}$ ); number of spikes within a burst; ISI inside a burst; inter-burst interval (IBI, s); and burst length (s).

\section{Data Segregation by PCA and K-Means Methods-NETCAL}

The nine neuronal features obtained from the activity analysis were transformed into a new basis by principal component analysis (PCA) [24]. The PCA data was then split into 8 significant groups through a k-means clustering algorithm [25]. The compound set of features of each of the groups was then further analysed.

\section{RNA Isolation and Retrotranscription}

Total RNA was isolated along the neuronal differentiation using TRI Reagent (TR118; Molecular Research Center; Ohio, USA) following the manufacturers' protocol. Ten 
microliters of total RNA at a concentration of $100-200 \mathrm{ng} / \mu \mathrm{l}$ ( $1 \mu \mathrm{g}$ in total) for each sample were reverse-transcribed with random primers using the high-capacity RNA-to-cDNA Kit (4387406, Thermo Fisher Scientific). Ten microliters of retrotranscription cocktail $(2 \mu \mathrm{l}$ of $10 \times$ retrotranscription (RT) buffer, $2 \mu \mathrm{l}$ of Random primers, $1 \mu \mathrm{l}$ of dNTP mix; $1 \mu \mathrm{l}$ MultiScribe reverse transcriptase) were added to each sample ( $20 \mu \mathrm{l}$ total volume). After gentle mixing, the samples were incubated for $10 \mathrm{~min}$ at room temperature followed by $2 \mathrm{~h}$ at $37^{\circ} \mathrm{C}, 10 \mathrm{~min}$ on ice and $10 \mathrm{~min}$ at $75^{\circ} \mathrm{C}$.

\section{High-Throughput Quantitative PCR-Openarray and Data Analysis}

Open array analysis was performed as described elsewhere [19]. Complementary cDNAs from 0 DIV to 37 DIV hPSCsamples were loaded onto the custom openarrays and run as recommended by the manufacturer on the QuantStudio $12 \mathrm{~K}$ Flex Real-Time PCR system (Thermo Fisher Scientific) by Servei Veterinari de Genètica Molecular (Faculty of Veterinary, Universitat Autònoma de Barcelona, Cerdanyola del Vallès, Spain). We ran two openarrays, the so-called developmental openarray with 112 TaqMan probes (Thermo Fisher Scientific), and a second openarray called Ephys with 168 TaqMan probes [19]. Both openarrays specifically detected all isoforms of each gene that were selected from the literature, and included 6 housekeeping genes for the developmental (18S, B2M, HPRT1, HSP90AB1, RPL13A, UBC) and 10 housekeeping genes for the Ephys (18S, B2M, HPRT1, HSP90AB1, RPL13A, UBC, PAPOLA, ACTB, EIF2B1, TBP) that were used as reference genes. For data analysis, Ctr-Q33 and GEN-Q18 samples were ran and analysed separately. For each openarray, we took one random sample from 37 DIV as reference sample. Relative gene expression was calculated using the Expression Suite Software 1.03 (Life Technology, Barcelona, Spain). Relative quantity (RQ) minimum and maximum values (error bars) were calculated with a confidential level of $95 \%$, using Benjamini-Hochberg false discovery rate to adjust $P$ values. Maximum allowed threshold cycle $\mathrm{Ct}$ included in calculations is 30 and a quantitation cycle $\mathrm{Cq}$ confidence $>0.8$. Error bars are presented in all graphs as standard error of the mean (SEM). Gene expression profile data are represented in graphics as RQ to 37 DIV.

\section{Protein Isolation and Western Blot}

Total protein extract was isolated from human tissue using TRI Reagent (T9424, Sigma-Aldrich, Madrid, Spain) according to the manufacturer's protocol. Total protein extracts were denatured using $1 \%$ SDS at $100{ }^{\circ} \mathrm{C}$ for $5 \mathrm{~min}$. Sixteen microgram of each denatured sample was subjected to $12 \%$ SDSPAGE and transferred to a nitrocellulose membrane (IPVH00010, Millipore, Barcelona, Spain) for $60 \mathrm{~min}$ at
$1 \mathrm{~mA} / \mathrm{cm}^{2}$. Membranes were incubated o.n. with primary antibodies (Ref. and dilution defined in Table 1) in immunoblot buffer (TBS-T-5\%M; Tris-buffered saline (TBS) containing $0.05 \%$ Tween- 20 and $5 \%$ no-fat dry milk) at $+4{ }^{\circ} \mathrm{C}$ in agitation and $1 \mathrm{~h}$ at RT upon a tilt table with HRP secondary antibodies in TBS-T-5\%M. For load control, anti-alpha actin $(1: 20,000)$ was incubated for $20 \mathrm{~min}$ at RT in TBS-T-5\%. Membrane development was performed in TBS-T-5\% BSA. Membranes were developed using the Luminata Classico or Forte Western HRP Substrate (WBLUC0100 and WBLUF0100, respectively, MERCK Millipore, Darmstadt, Germany) in Fuji Medical X-Ray Films (Super RX-N; Fujifilm Co., Tokyo, Japan).

\section{Statistical Analysis}

For comparisons between 16 DIV Ctr-Q33 and GEN-Q18 NPCs, two-way ANOVA followed by Tukey's multiple comparison test was applied and values of $P<0.05$ were considered statistically significant, $* P<0.05, * * P<0.005$, $* * * P<$ 0.0005 . Neuronal progenitor cells were represented as relative to total labelled nuclei or relative to PAX6 NPCs in double labelling. Error bars were presented in all graphs as standard error of the mean (SEM).

For comparisons between Ctr-Q33 and GEN-Q18 neuronal cell types at 23 DIV and 37 DIV, two-way ANOVA followed by Tukey's multiple comparison test was applied, $* P<0.05$, $* * P<0.005, * * * P<0.0005$. Neuronal cell types were represented as relative to total labelled nuclei by DAPI immunofluorescence or relative to Map 2b. Error bars were presented in all graphs as SEM.

For gene expression comparisons along the differentiation from 0 DIV to 37 DIV, one-way ANOVA followed by Tukey's multiple comparison test was applied. Relative to 0 DIV, $* P<0.05, * * P<0.01$, $* * * P<0.001$; to DIV $8+P<$ $0.05,++P<0.01,+++P<0.001 ; 12$ DIV \# $P<0.05$, \#\# $P<$ 0.01 , \#\#\# $P<0.001$; to DIV $16 \$ \$ P<0.01, \$ \$ \$ P<0.001$; to 23 DIV $\& P<0.05$, \&\& $P<0.01$ and $\& \& \& P<0.001$. Error bars were presented in all graphs as SEM. Gene expression profile data were represented in graphics as RQ to 37 DIV.

For firing and bursting feature comparisons between groups, G1 to G8 at 37 DIV, one-way ANOVA followed by Tukey's multiple comparison test was applied. $P<0.05^{*}, P \leq$ $0.01 * *$ and $P \leq 0.001 * * * . P>0.05$. Data is summarized in Fig. S12 and grey boxes).

Colour matrices represented all possible comparisons on the diagonal (blue box; 0 DIV to 37 DIV) on which the statistical test was carried out for all the multiple comparisons. Non-expressing genes were indicated with grey boxes. Colour boxes for expressing genes with $P<0.05$. Upregulated (red) and downregulated (green) genes were represented with increasing three colour-scale intensities for $P \leq 0.5, P \leq 0.005$ and $P \leq 0.0005$, respectively. 
Protein quantification of Ctr-Q33 and GEN-Q18 at DIV 0, DIV 23 and DIV 37 was calculated by relative quantity to alphaactin band. One-way ANOVA followed by Tukey's multiple comparison test was applied and values of $P<0.05$ were considered statistically significant. Relative to $0 \mathrm{DIV}, * P<0.05$, $* * P<0.005$, *** $P<0.0005$; to DIV $23 \# P<0.05, \#+P<$ 0.01 , \#\# $P<0.001$. Protein levels were represented in graphics as relative protein to alpha-actin and error bars as SEM.

\section{Quality System}

All procedures were conducted under UNE-ENISO9001:2015 and comply with the Guidance Document on Good In Vitro Method Practices (GIVIMP; OECD) recommendations [26].

\section{Results}

Two sources of hPSC cell lines, an induced Ctr-Q33 and an embryonic GEN-Q18 hPSCs, successfully differentiated into forebrain mature neurons in 37 DIV. The in vitro neuronal differentiation comprised 3 successive stages illustrated in Fig. 1. First, the protocol consisted in the neural induction and expansion of progenitor cells during the first 8 DIV. Second, neuroectodermal (NE) progenitors were regionalized into anterior forebrain or telencephalic NPCs at 16 DIV. And, third, the protocol promoted hPSC-derived NPCs cell cycle exit together with terminal neurogenesis and synaptogenesis until 37 DIV.

All gene and protein nomenclatures are detailed in Table S1.

\section{The Synergetic Dual-SMAD and WNT Inhibition Forces hPSCs to Exit from Pluripotency}

At 0 DIV, both hPSC cell lines are arranged in monolayer colonies throughout the culture. HPSC colonies were entirely composed by pluripotent cells positive for OCT-3/4 TF (Fig. S1 $\mathrm{a}$ and $\mathrm{b}$ and panels $\mathrm{a}$ ' and $\mathrm{b}$ '). HPSCs began to differentiate by using dual-SMAD inhibition along with $\mathrm{Wnt} / \beta$-catenin inhibitor (SLI media; neural induction Fig. 1). By means of quantitative gene expression analysis, we observed that both hPSC cell lines downregulated the expression of well-known pluripotent TFs POU5F1 (OCT4), NANOG and KRT18 (Fig. $\mathrm{S} 1$ panels $\mathrm{c}$ and $\mathrm{d}$ ).

\section{HPSC-Derived Neuroepithelial Progenitors Homogeneously Neuralize in Bi-dimensional Neural Tube-like Structures After 8 DIV}

For neural fate specification and neurulation of hPSC, fourth additional days of BMP, TGF $\beta$ and $\mathrm{Wnt} / \beta$-catenin inhibitors were applied to hPSC-derived cultures from 4 DIV to $8 \mathrm{DIV}$
(Fig. 1; neural induction). HPSCs successfully acquired a neuroepithelial (NE) progenitor identity at 8 DIV. These NE progenitors are self-arranged in bi-dimensional neural tube-like or rosette structures (Fig. 2). Moreover, hPSC-derived NE progenitors expressed neurulation proteins $\mathrm{ZO}$, tight junction protein 1 (TJP1) and PLZF (promyelocytic leukaemia zinc finger) at protein and mRNA levels. Representative images of Ctr-Q33 and GEN-Q18 hPSC-derived NE cultures are shown in Fig. 2a and Fig. 1b, respectively. As expected, PLZF is expressed within the nucleus of NE progenitors (Fig. 2a and b), and ZO1 is expressed apically at the luminal zone of the rosettes (Fig. 2a and $b$ ). The acquisition of neural fate by hPSC-derived NE progenitors was also corroborated by gene expression profile (Fig. $2 \mathrm{c}$ and $\mathrm{d})$. The Zing finger and BTB domain containing 16 (ZBTB16/ PLZF) gene were induced at 8 DIV by Ctr-Q33 and GEN-Q18 NE progenitors. In contrast, TJP1/ ZO1 was found throughout the neuronal differentiation but its expression was downregulated at late differentiation stages.

Additionally, Ctr-Q33 and GEN-Q18 hPSC-derived NE cultures induced the expression of early NPC-related genes (Fig. 3a and b, respectively). HPSC-derived NE progenitors upregulated the cytoskeleton early neural gene nestin (NES) from 8 DIV until 16 DIV. OTX-1, -2 and SOX2 genes were upregulated from 8 DIV and became downregulated at late differentiation stages (37 DIV). Neural identity of Ctr-Q33 and GEN-Q18 hPSC-derived cultures was corroborated by immunolabelling for NES and $\beta$-III tubulin. At 12 DIV, CtrQ33 cultures were mainly composed by nestin-positive NPC along with few progenitors positive for the neuronal marker $\beta$-III tubulin (Fig. 3c).

\section{Regionalization of hPSC-Derived NPCs Gives Rise to Mainly Subpallial Telencephalic Progenitor at 16 DIV}

We next assessed by quantitative gene expression analysis the regionalization of hPSC-derived NPCs. Figure S2 shows the consolidation of the anterior commitment of Ctr-Q33 and GEN-Q18 hPSC-derived NPCs (S2a and b, respectively). Both cell lines induced the expression of the forebrain FOXG1, DACH, SIX3 and GLI3genes at 16 DIV. We neither detected expression of the midbrain PAX2, hindbrain HOXB 4/9 nor the spinal cord FOXA2A region-specific genes along the neuronal differentiation (data not shown). From 8 DIV to 16 DIV, Ctr-Q33 and GEN-Q18 cultures progressed towards the neuronal differentiation by expressing the neuronal genes DCX, TUBB3 and NCAM1 (doublecortin, $\beta$-III tubulin and neural cell adhesion molecule respectively; Fig. S3).

In order to distinguish among the different NPC subtypes, we quantified the proportions of dorsal PAX $6^{+}$NPCs and ventral DLX_pan ${ }^{+}$and early B cell factor $1(\mathrm{EBF} 1)^{+} \mathrm{NPCs}$ present at 16 DIV (Fig. 4a). Ctr-Q33 and GEN-Q18 cultures contained similar proportions of ventral $\mathrm{DLX}^{+}$and $\mathrm{EBF}^{+}$ 


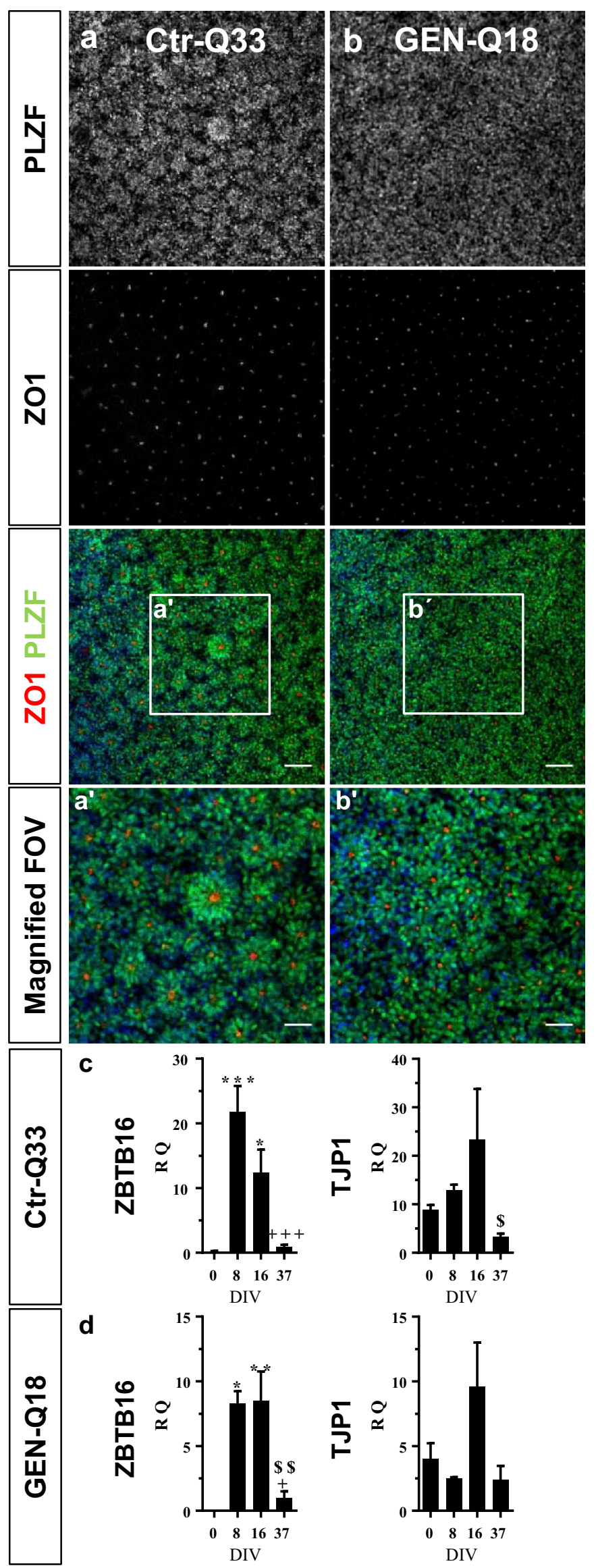


Fig. 2 Immunostaining of neural tube-like markers, PLZF (green) and ZO1 (red) in a Ctr-Q33 and b GEN-Q18 hPSC-derived NE progenitors at DIV 8. The squared boxes ( $a^{\prime}$ and $b^{6}$ ) show a magnified field of view (FOV) from a and $\mathbf{b}$. Scale bar $50 \mu \mathrm{m}$. Quantitative gene expression of ZBTB16/PLZF and TJP1/ZO1 along e Ctr-Q33 and d GEN-Q18 neuronal differentiation. (Mean \pm SEM; one-way ANOVA followed by Tukey's multiple comparison test, relative to 0 DIV $* P<0.05$, $* * P<$ 0.01 , *** $P<0.001$; to $8+$ DIV $P<0.05,++P<0.01,+++P<0.001$; to 16 DIV $\$ \$ P<0.01, \$ \$ \$ P<0.001)$

NPCs (Fig. 4b). For DLX_pan NPCs, Ctr-Q33-derived cultures contained $42 \pm 2 \%$ and GEN-Q18-derived cultures $39 \pm$ $3 \%$. For EBF1, the proportions were slightly lower compared with DLX pan in both cell lines, $24 \pm 1 \%$ and $26 \pm 1 \%$ for Ctr-Q33 and GEN-Q18, respectively. For dorsal progenitors, GEN-Q18 cultures contained more PAX6 ${ }^{+}$NPCs compared with Ctr-Q33 cultures at 16 DIV with $45 \pm 2 \%$ and $26 \pm 2 \%$, respectively (Fig. 4b). However, since PAX6 besides specify dorsal NPCs is also expressed by NE progenitors [27], we discriminated between $\mathrm{PAX}^{+}$early NE progenitors and pallial-specific PAX6 ${ }^{+}$NPCs. To do so, we performed double labelling of dorsal and ventral markers (Fig. 4d and e). From the entire PAX6 population, most $\mathrm{PAX}^{+}{ }^{+} \mathrm{NPCs}$ co-labelled with ventral markers. In Ctr-Q33-derived NPC cultures, $47 \%$ \pm 3 PAX6 progenitors co-labelled with DLX_pan and $41 \pm 5 \%$ with EBF1 (Fig. 4c). In GEN-Q18-derived NPC cultures, 28 $\pm 2 \%$ of PAX6 progenitors co-labelled with DLX_pan and 36 $\pm 1.3 \%$ with EBF1 (Fig. 4c). Representative images from the double-labelled dorsal and ventral NPCs in Ctr-Q33 and GEN-Q18 at DIV 16 are illustrated in Fig. 4d and e, respectively.

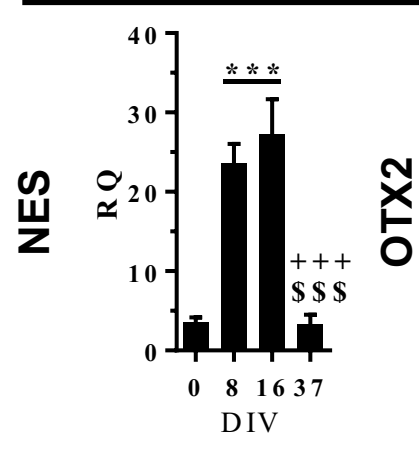

b

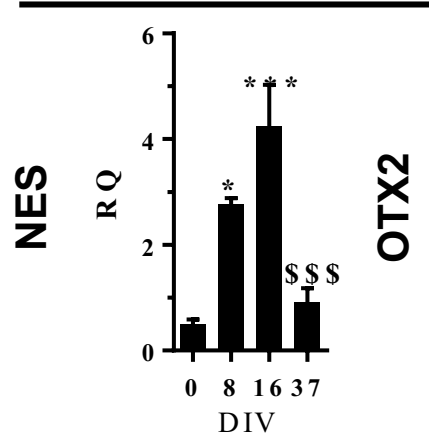

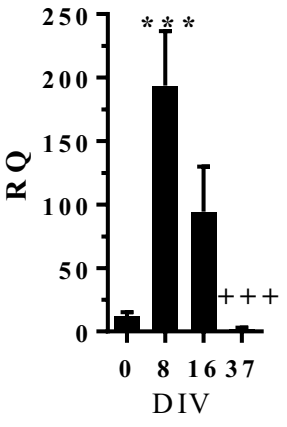

GEN-Q18
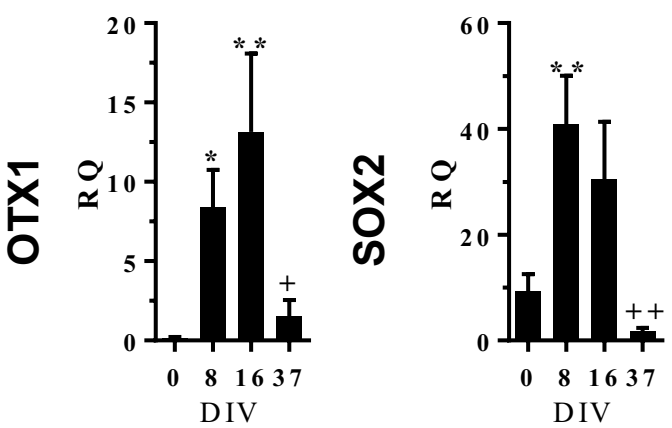

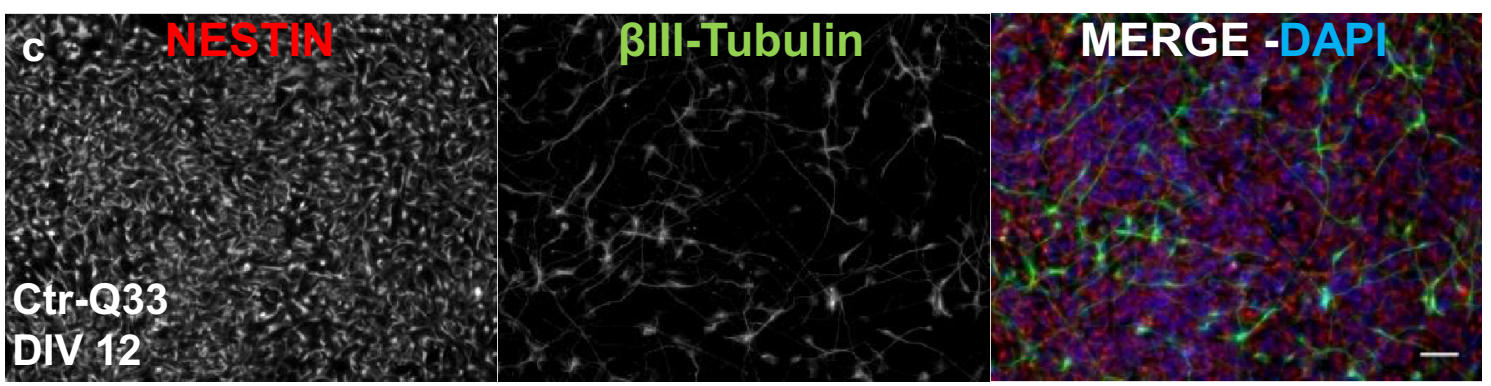

Fig. 3 Quantitative gene expression of neural NES, OTX-2/1 and SOX2 genes by a Ctr-Q33 and b GEN-Q18 hPSC-derived cultures along the neuronal differentiation. (Mean $\pm \mathrm{SEM}$; one-way ANOVA followed by Tukey's multiple comparison test, relative to 0 DIV $* P<0.05$, $* * P<$ $0.01, * * * P<0.001$; to 8 DIV $+P<0.05,++P<0.01,+++P<0.001$; to
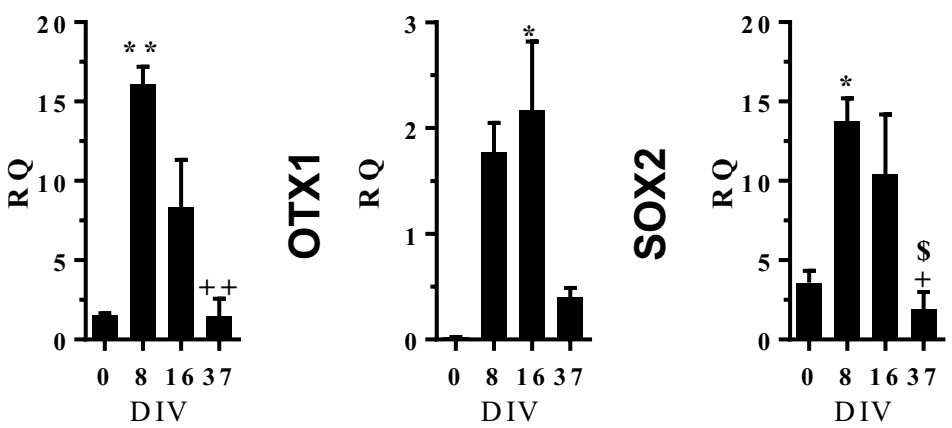

16 DIV $\$ \$ P<0.01, \$ \$ \$ P<0.001)$. c Immunofluorescence for the early NPC marker nestin (red) and the neuronal progenitor marker $\beta$-III tubulin (green) in Ctr-Q33 hiPSC-derived NPC cultures at DIV 12. Scale bar $50 \mu \mathrm{m}$ 

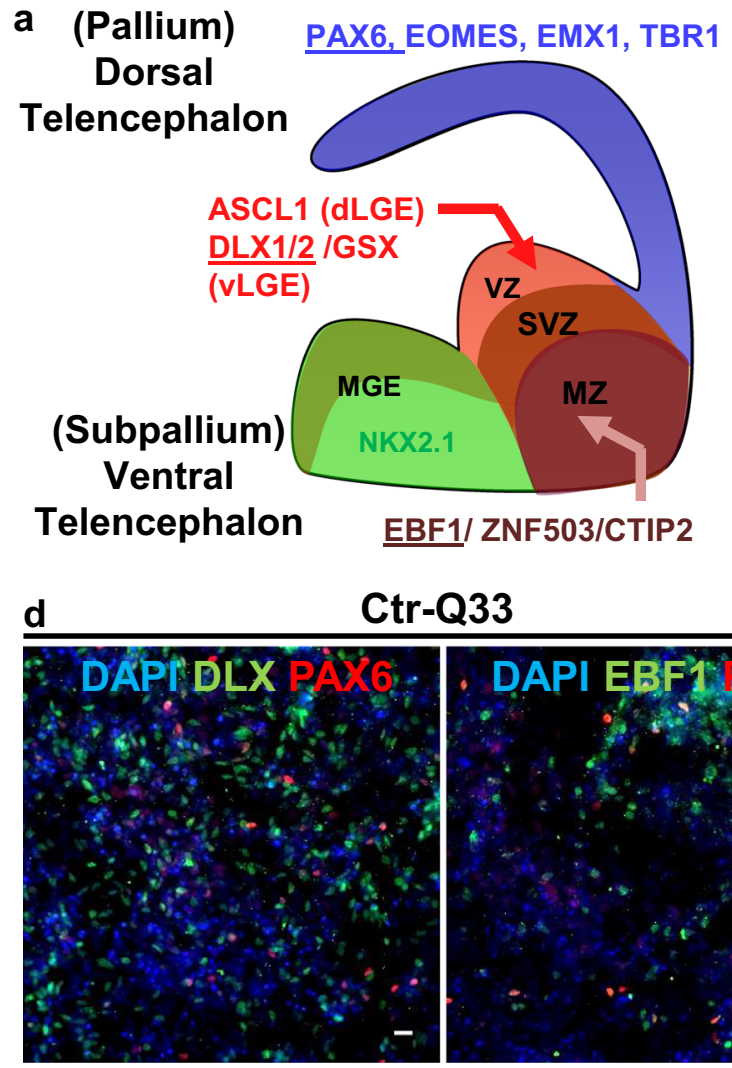

Ctr-Q33

Fig. 4 Ctr-Q33 and GEN-Q18 hPSC-derived NPC's populations at 16 DIV. a Schematic representation of pallial and subpallial telencephalic region-specific TFs. b Proportion of PAX6+, DLX_pan+ and EBF1+ NPCs relative to total cells in Ctr3-Q33 (black) and GEN-Q18 (grey)derived NPC cultures. $\mathbf{c}$ Proportion of ventral DLX_pan+ and EBF1+

Furthermore, we analysed the most relevant TFs involved in telencephalic specification. The differentiation of hPSC resembled endogenous striatal neural differentiation. From 12 DIV to 16 DIV, cells upregulated the expression of LGE VZrelated GSX2 and GSX1 genes which were afterwards downregulated from 23 DIV onwards (Fig. 5a). The LGE VZrelated ASCL1 gene displayed a single-induction peak at 16 DIV and thereafter, it became downregulated from 23 DIV onwards (Fig. 5a and S4a, for Ctr-Q33 and GEN-Q18, respectively). Interestingly, differentiated hPSC displayed a gradual expression of DLX genes from 16 DIV onwards (Fig. 5b). HPSC-derived NPCs induced the expression of the SVZrelated DLX2 and DLX1 at 16 DIV (Fig. 5b and S4b) followed by the induction of the striatal MZ-related DLX5 and DLX6 genes along with EBF1 from 23 DIV (Fig. 5c and S4c). As expected, the expression of PAX6 was found early from $8 \mathrm{DIV}$, to specify neural fate, to $16 \mathrm{DIV}$ (Fig. 5d and S4d). Thereafter, the cortical SVZ-related EOMES displayed a single-induction peak at 12 DIV and became downregulated afterwards until the end of the differentiation. Finally, hPSCderived cultures upregulated the cortical plate (CP)-related TBR1 gene at 16 DIV (Fig. $5 \mathrm{f}$ and S4f).
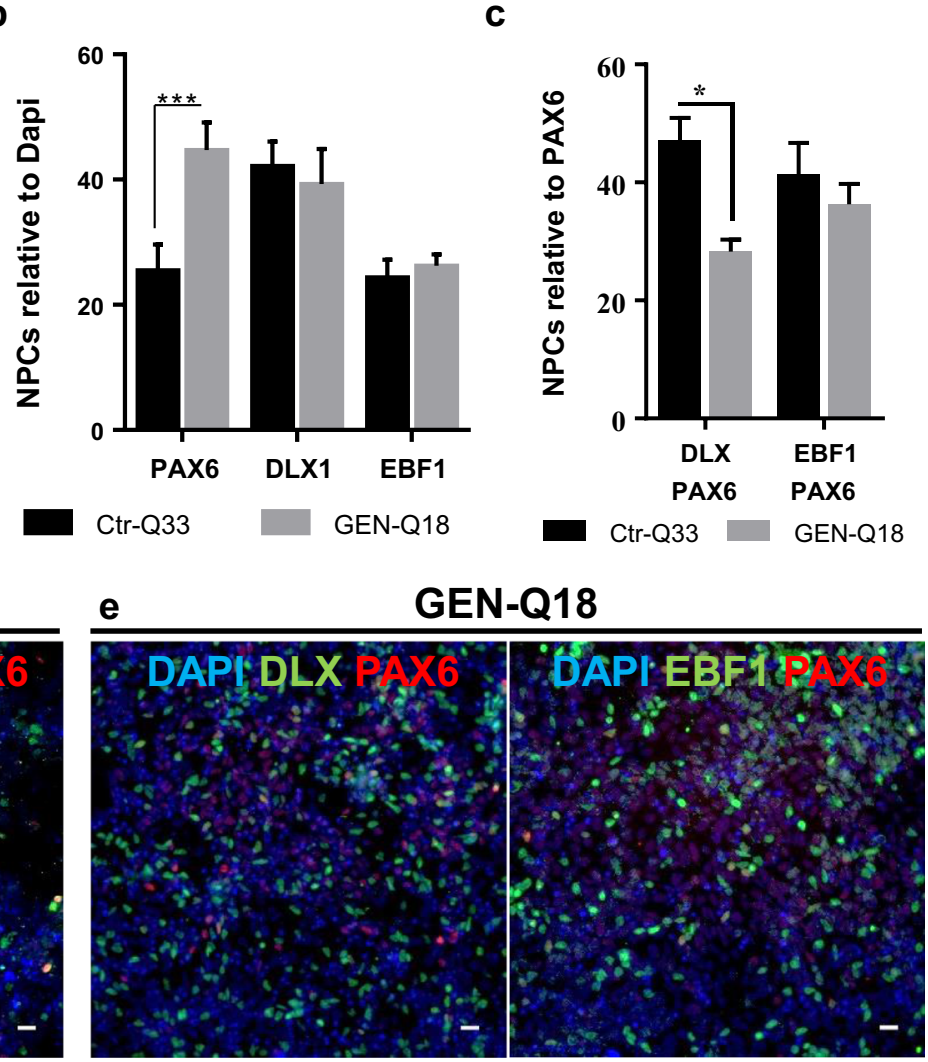

NPCs double labelled with dorsal PAX6 progenitors. (Mean $\pm \mathrm{SEM}$; two-way ANOVA followed by Tukey's multiple comparison test, $* P<$ $0.05, * * P<0.005, * * * P<0.0005)$. Representative images of doublelabelled PAX6 (red) with DLX_pan or EBF1 (green) in d Q33n1 and e GEN-Q18 hPSC-derived cultures at 16 DIV. Scale bar $100 \mu \mathrm{m}$

\section{HPSC-Derived Neurons Reached Synaptic Maturity at 23 DIV}

HPSC-derived NPCs differentiated and generated postmitotic neuronal cultures (Fig. 6). Quantitative gene expression of mature neuronal genes MAP2 and RBFOX3 (NeuN) were induced by both hPSC cell lines starting at 16 DIV (Fig. 6a and b). Ctr-Q33 and GEN-Q18 cell lines gave rise to almost $100 \%$ of Map2b neurons from 23 DIV onwards, without differences between cell lines at 23 DIV and 37 DIV (for CtrQ33, 97\% and 95\%, and for GEN-Q18, 99\% and 98\% at 23 DIV and 37 DIV, respectively; Fig. 6c). Consistently, we observed that hPSC-derived neurons displayed neurites outgrowth and abundant arborisations as differentiation progressed (Fig. 6d and e).

To confirm whether hPSC-derived neurons achieved synaptic maturation after neuronal differentiation, we analysed the expression of synaptic-related markers at protein (Fig. 7) and their gene expression levels (Fig. 8). HPSC-derived neurons expressed the soluble NSF attachment protein (SNA) REceptor (SNARE) proteins synaptotagmin, syntaxin-1 and synaptosomal-associated protein 25 (SNAP-25) at 23 DIV and 


\section{Subpallial genes}

\section{Pallial genes}
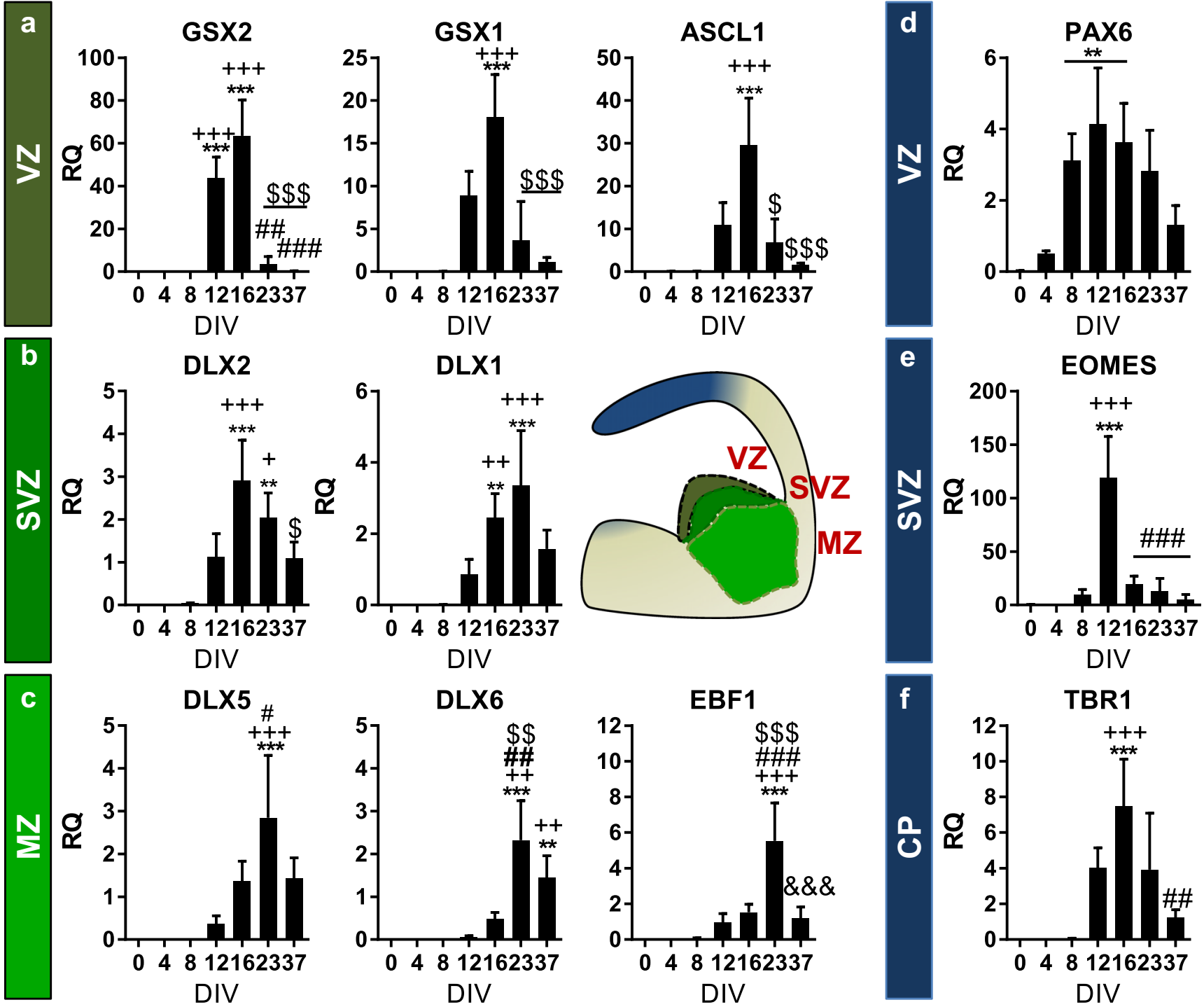

Fig. 5 Quantitative gene expression of $\mathbf{a}-\mathbf{c}$ telencephalic subpallial and d-f pallial gene expression throughout Ctr-Q33 neuronal differentiation. (Mean \pm SEM; one-way ANOVA followed by Tukey's multiple comparison test, relative to 0 DIV $* P<0.05, * * P<0.01$, $* * * P<$ 0.001 ; to $8 \mathrm{DIV}+P<0.05,++P<0.01,+++P<0.001$; to $12 \mathrm{DIV} \#$

$P<0.05$, \#\# $P<0.01$, \#\#\# $P<0.001$; to 16 DIV $\$ P<0.05$, $\$ \$ P<0.01$, $\$ \$ \$ P<0.001$; to 23 DIV \& $P<0.05, \& \& P<0.01, \& \& \& P<0.001) . V Z$ ventricular zone; $S V Z$ subventricular zone; $M Z$ mantel zone and $C P$ cortical plate

37 DIV. All three SNAREs were largely expressed at 37 DIV compared with 23 DIV (Fig. 7a and b). SNAP25 was also detected by immunofluorescence along with the postsynaptic NMDAR subunit 1 (NR1) at 37 DIV (Fig. 7c). Ctr-Q33derived neurons displayed abundant punctate SNAP-25 staining throughout the cytosol and projections of which some SNAP-25 punctuates co-localized with NR1, as shown in Fig. 7d.

We next performed a wide screening of a set of presynaptic and postsynaptic genes along the Ctr-Q33 hiPSC neuronal differentiation (Fig. 8). Ctr-Q33-derived neurons upregulated the expression of presynaptic endocytosis-related protein BIN1 from DIV 16. Synaptotagmin V (SYT5), synapsin I (SYN1), vesicle-associated membrane protein 2 (VAMP2)

and synaptic vesicle glycoprotein 2C (SV2C) genes were found induced from 23 DIV onwards (Fig. 8a). At 37 DIV, Ctr-Q33 neurons induced the gene expression of SNAP25 and synaptophysin (SYP). Conversely, the neuronal differentiation resulted in the downregulation of SYT2, VAMP5 and SYNJ2 (Fig. 8a). Regarding postsynaptic genes, Ctr-Q33 neurons showed a significant upregulation DLG2 (PSD-93; Discs large homologues 2/postsynaptic density protein 93) at 37 DIV (Fig. 8b). Other postsynaptic proteins, such as DLG1/SAP97, DLG3/SAP102 and DLG4//PSD95, were expressed along Ctr-Q33 hiPSC differentiation but they did not show significant differences between DIVs (Fig. S17a). 
a

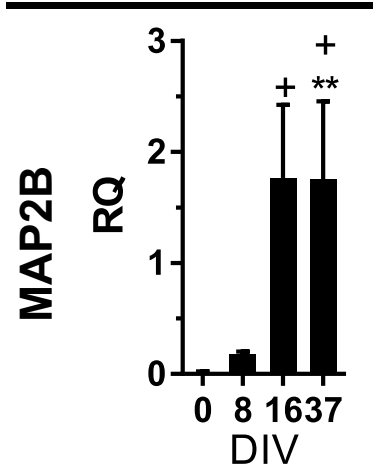

C

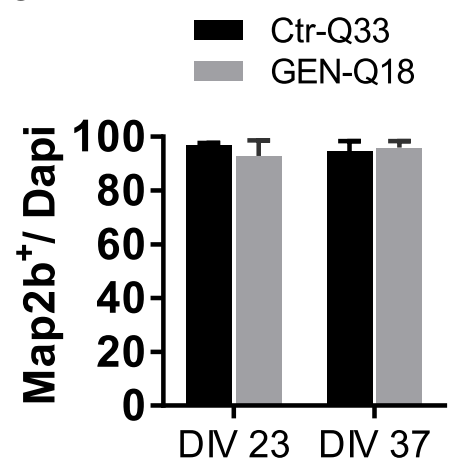

b

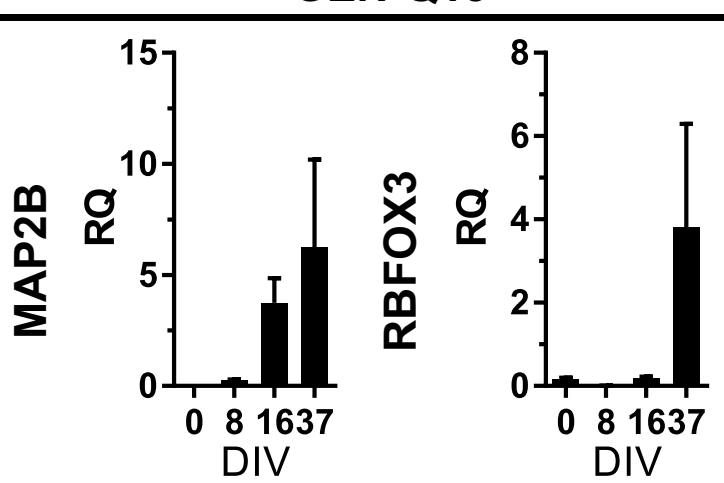

d

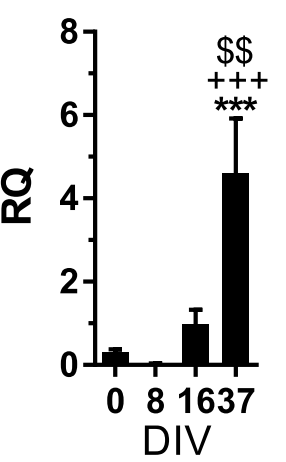

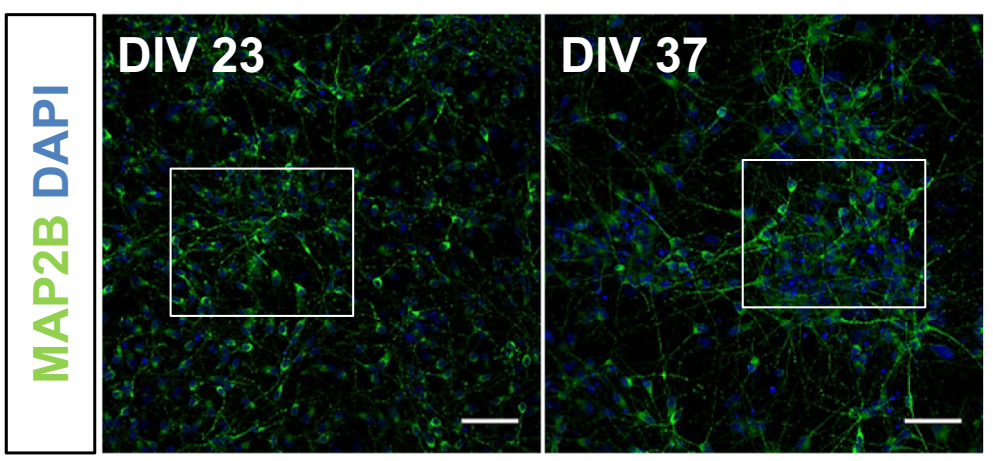

e

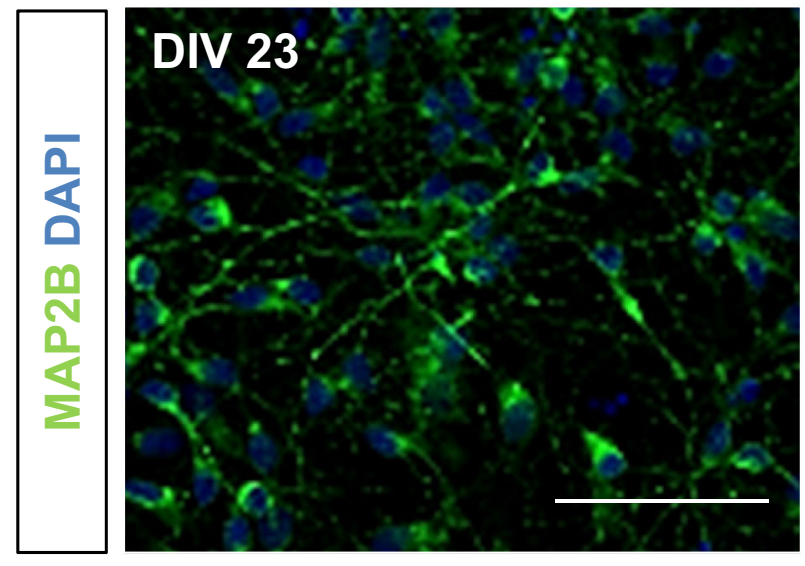

Fig. 6 Differentiation of Ctr-Q33 and GEN-Q18 hPSC generates MAP2b neurons from 23 DIV onwards. Gene expression profile of MAP2 and RBFOX3 along a Ctr-Q33 and b GEN-Q18 neuronal differentiation (Mean \pm SEM; one-way ANOVA followed by Tukey's multiple comparison test, relative to 0 DIV $* P<0.05, * * P<0.01$, $* * * P<$ 0.001 ; to $8 \mathrm{DIV}+P<0.05,++P<0.01,+++P<0.001$; to $16 \mathrm{DIV} \$$

\section{Neuronal Cultures Comprise GABA, TH and TBR1-Expressing Neurons from 23 DIV}

We performed a detailed characterization of the main forebrain neuronal cell types, including GABAergic, glutamatergic, dopaminergic (DAergic) -tyrosine hydroxylase $(\mathrm{TH})^{+}$ neurons and MSN-like neurons, in Ctr-Q33 and GEN-Q18 hPSC-derived neuronal cultures.
$P<0.05, \$ \$ P<0.01, \$ \$ \$ P<0.001)$. c Proportion of MAP2b + neurons by Ctr-Q33 (black) and GEN-Q18 (grey) at 23 and 37 DIV (Mean \pm SEM; two-way ANOVA followed by Tukey's multiple comparison test; $P<0.05)$. d Image of MAP2b expression by Ctr-Q33 hiPSC-derived neurons at DIV 23 and 37. e High-magnification images from insets in

d. Scale bar $10 \mu \mathrm{m}$

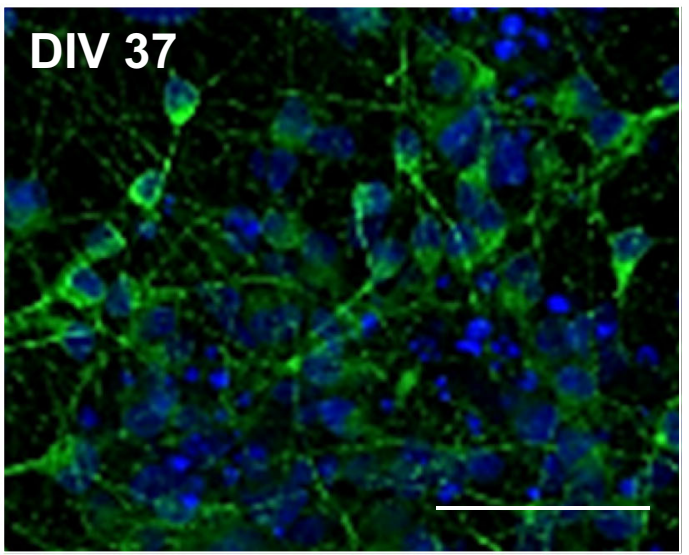

Ctr-Q33 and GEN-Q18-derived neuronal cultures contained a high amount of $\mathrm{GABA}^{+} / \mathrm{Map}^{2} \mathrm{~b}^{+}$neurons at 23 DIV and 37 DIV (Fig. 9; Tables 2 and 3). Ctr-Q33-derived neuronal cultures showed a tendency to decrease the number of $\mathrm{GABA}^{+}$population from $50 \pm 8 \%$ at $23 \mathrm{DIV}$ to $30 \pm 6 \%$ at 37 DIV, whereas GEN-Q18-derived neuronal cultures showed a slight increase from $38 \pm 7 \%$ at 23 DIV to $47 \pm 7 \%$ at 37 DIV (Fig. 9a). Representative immunofluorescence images for 
a

Ctr-Q33

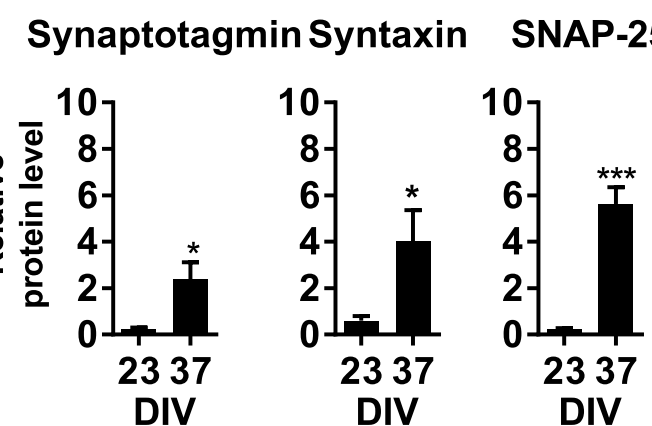

b

GEN-Q18

\section{Synaptotagmin Syntaxin SNAP-25}
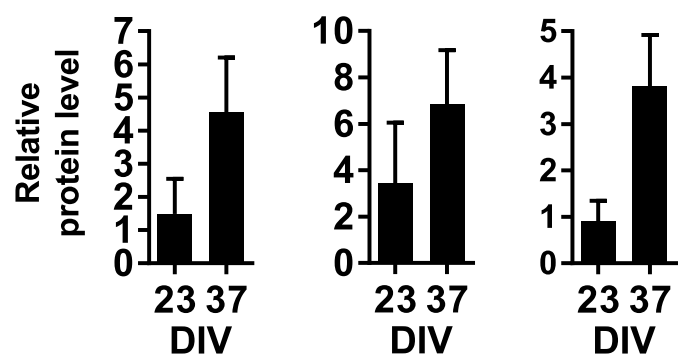

Synapto.

Syntaxin1

SNAP-25

$\alpha-A c t i n$
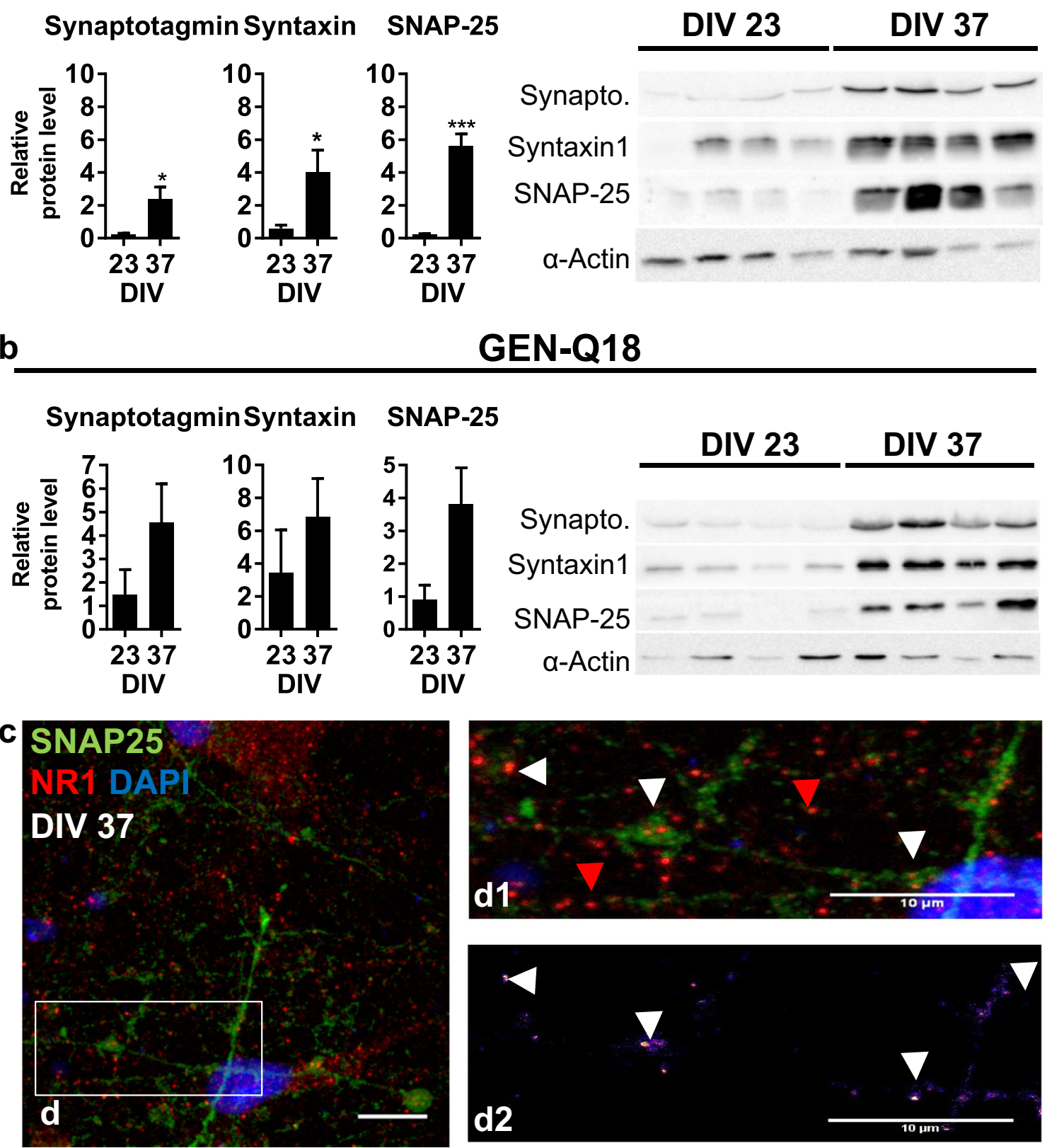

Fig. 7 Expression of SNARE proteins by a CTR-Q33 and b GEN-Q18 from 23 DIV onwards. Relative quantity to alpha-actin. (Unpaired $t$ test with Welch's correction, relative to 23 DIV $* P<0.05$, $* * P<0.005$ and $* * * P<0.001$; Mean \pm SEM). c Staining of SNAP-25 (red) and NR1 (green) in Ctr-Q33 hiPSC-derived neurons at DIV 37 with the colocalization punctate in the magnified FOV b'1 and 2. White arrowhead indicates NR1-SNAP-25 colocalization punctate; red arrowhead single indicates SNAP-25 punctate. Scale bar $10 \mu \mathrm{m}$ these time points are shown in Fig. 9b for Ctr-Q33 and Fig. S5 for GEN-Q18, highlighting the increase of $\mathrm{GABA}^{+}$projections, both in number and length, from 23 DIV to 37 DIV. GABAergic neuronal differentiation was also corroborated by quantitative analysis of the expression of a set of GABAergic-related genes (Fig. 9c for Ctr-Q33 and Fig. 9d for GEN-Q18). The cultures had already upregulated the expression of GAD2,
CALB2 (calretinin) and SST (somatostatin) and the interneuron-specific LHX1 (LIM homeobox 1) gene at 16 DIV.

Concerning DAergic- $\mathrm{TH}^{+}$neurons, GEN-Q18 cultures at 23 DIV contained $50 \pm 2 \% \mathrm{TH}^{+} / \mathrm{Map}^{2} \mathrm{~b}^{+}$neurons, a much higher population than the $19 \pm 2 \%$ of Ctr-Q33 cultures (Fig. 10a). As maturation progressed, however, the proportion of $\mathrm{TH}^{+}$neurons among lines reached similar values, with $36 \pm$ 
a

\begin{tabular}{|c|c|c|c|c|c|c|}
\hline \multicolumn{7}{|c|}{ SYT2 } \\
\hline DIV & 0 & 4 & 8 & 12 & 16 & 23 \\
\hline 0 & 0 & & & & & \\
\hline 4 & & 4 & & & & \\
\hline 8 & & & 8 & & & \\
\hline 12 & & $\mathrm{~ns}$ & & 12 & & \\
\hline 16 & & ns & & ns & 16 & \\
\hline 23 & ns & ns & & ns & ns & 23 \\
\hline 37 & $\mathrm{~ns}$ & $\mathrm{~ns}$ & & ns & ns & ns \\
\hline
\end{tabular}

\begin{tabular}{|c|c|c|c|c|c|c|}
\hline \multicolumn{10}{|c|}{ SYP } \\
\hline DIV & $\mathbf{0}$ & $\mathbf{4}$ & $\mathbf{8}$ & $\mathbf{1 2}$ & $\mathbf{1 6}$ & $\mathbf{2 3}$ \\
\hline $\mathbf{0}$ & $\mathbf{0}$ & \multicolumn{7}{|c|}{} \\
\hline $\mathbf{4}$ & ns & 4 & \multicolumn{7}{|c|}{} \\
\hline $\mathbf{8}$ & ns & ns & $\mathbf{8}$ & \multicolumn{3}{|c|}{} \\
\hline $\mathbf{1 2}$ & ns & ns & ns & 12 & \multicolumn{1}{|c|}{} \\
\hline 16 & ns & ns & ns & ns & 16 & \\
\hline $\mathbf{2 3}$ & ns & ns & ns & ns & ns & $\mathbf{2 3}$ \\
\hline 37 & & & & & & ns \\
\hline
\end{tabular}

\begin{tabular}{|c|c|c|c|c|c|c|}
\hline & \multicolumn{6}{|c|}{ VAMP5 } \\
\hline DIV & 0 & 4 & 8 & 12 & 16 & 23 \\
\hline 0 & 0 & & & & & \\
\hline 4 & & 4 & & & & \\
\hline 8 & & $\mathrm{~ns}$ & 8 & & & \\
\hline 12 & & ns & $\mathrm{ns}$ & 12 & & \\
\hline 16 & & ns & $\mathrm{ns}$ & $\mathrm{ns}$ & 16 & \\
\hline 23 & $\mathrm{~ns}$ & $\mathrm{~ns}$ & $\mathrm{~ns}$ & $\mathrm{~ns}$ & $\mathrm{~ns}$ & 23 \\
\hline 37 & ns & $\mathrm{ns}$ & $\mathrm{ns}$ & $\mathrm{ns}$ & $\mathrm{ns}$ & \\
\hline
\end{tabular}

\begin{tabular}{|c|c|c|c|c|c|c|}
\hline \multicolumn{8}{|c|}{ SYT5 } \\
\hline DIV & 0 & 4 & 8 & 12 & 16 & 23 \\
\hline 0 & 0 & \multicolumn{1}{|c|}{} \\
\hline
\end{tabular}

\begin{tabular}{|c|c|c|c|c|c|c|}
\hline & & & & & & \\
\hline 0 & 0 & & & & & \\
\hline 4 & ns & 4 & & & & \\
\hline 8 & ns & $\mathrm{ns}$ & 8 & & & \\
\hline 12 & ns & ns & ns & 12 & & \\
\hline 16 & ns & ns & ns & ns & 16 & \\
\hline 23 & & & & & & 23 \\
\hline 37 & & & & & & $\mathrm{~ns}$ \\
\hline
\end{tabular}
Presynaptic

\begin{tabular}{|c|c|c|c|c|c|c|}
\hline & & & & & & \\
\hline DIV & 0 & 4 & 8 & 12 & 16 & 23 \\
\hline 0 & $\overline{0}$ & & & & & \\
\hline 4 & $\mathrm{~ns}$ & 4 & & & & \\
\hline 8 & ns & ns & 8 & & & \\
\hline 12 & ns & $\mathrm{ns}$ & ns & 12 & & \\
\hline 16 & & & & ns & 16 & \\
\hline 23 & & & & $\mathrm{~ns}$ & $\mathrm{~ns}$ & 23 \\
\hline 37 & & & & $\mathrm{~ns}$ & ns & ns \\
\hline
\end{tabular}

\begin{tabular}{|c|c|c|c|c|c|c|}
\hline & \multicolumn{6}{|c|}{ SV $2 C$} \\
\hline DIV & 0 & 4 & 8 & 12 & 16 & 23 \\
\hline 0 & 0 & & & & & \\
\hline 4 & & 4 & & & & \\
\hline 8 & & & 8 & & & \\
\hline 12 & & & $\mathrm{~ns}$ & 12 & & \\
\hline 16 & & & $\mathrm{~ns}$ & ns & 16 & \\
\hline 23 & & & & & & 23 \\
\hline 37 & & & $\mathrm{~ns}$ & ns & ns & $\mathrm{ns}$ \\
\hline
\end{tabular}

Fig. 8 Quantitative gene expression of presynaptic and postsynaptic genes along Ctr-Q33 hiPSC neuronal differentiation. Matrices (DIV vs. DIV) for a presynaptic and b postsynaptic genes along Ctr-Q33 hiPSC neuronal differentiation. One-way ANOVA followed by Tukey's multiple comparison test was performed between DIVs (0 to 37). Coloured matrices represent all possible comparisons on the diagonal (blue box;

$5 \%$ for Ctr-Q33 and $52 \pm 4 \%$ for GEN-Q18 at 37 DIV (Fig. 10a; Tables 2 and 3). Representative immunofluorescence images for TH and MAP2b at DIV 23 and 37 DIV are shown in Fig. 10b for Ctr-Q33 and in Fig. S6a for GEN-Q18. In both cases, the length of $\mathrm{TH}^{+}$projections substantially increased from 23 DIV to 37 DIV. These data were corroborated by the induction of DAergic-related genes, NR4A2 (nuclear receptor subfamily 4 group A member 2), TH and LMX1B (LIM homeobox transcription factor 1 beta) from 16 DIV onwards (Fig. 10e-g for Ctr-Q33 and Fig. 10h and i for GEN-Q18). Many of the $\mathrm{TH}^{+}$neurons were also found to express GABA (Fig. S7), suggesting that a substantial proportion of $\mathrm{TH}^{+}$neurons is striatal interneurons which increase $\mathrm{TH}$ expression as neuronal differentiation proceeds.

We also observed that hPSC-derived neuronal cultures contained a low proportion of TBR $1^{+}$neurons. Both cell lines exhibited less than $7 \%$ of TBR $1^{+}$neurons in the entire Map2b + population (Fig. 10c; Tables 2 and 3). Ctr-Q33 hiPSC-derived neuronal cultures contained $4.0 \pm 0.3 \%$ of $\mathrm{TBR}^{+}{ }^{+}$neurons at $23 \mathrm{DIV}$, which increased to $7.0 \pm 0.4 \%$ at $37 \mathrm{DIV}$. In

\begin{tabular}{|c|c|c|c|c|c|c|c|c|c|c|c|c|c|}
\hline & & & & $\mathrm{NJ} 2$ & & & & & & SNA & P25 & & \\
\hline DIV & 0 & 4 & 8 & 12 & 16 & 23 & DIV & 0 & 4 & 8 & 12 & 16 & 23 \\
\hline 0 & 0 & & & & & & 0 & 0 & & & & & \\
\hline 4 & & 4 & & & & & 4 & ns & 4 & & & & \\
\hline 8 & $\mathrm{~ns}$ & & 8 & & & & 8 & $\mathrm{~ns}$ & $\mathrm{~ns}$ & 8 & & & \\
\hline 12 & & $\mathrm{~ns}$ & $\mathrm{~ns}$ & 12 & & & 12 & $\mathrm{~ns}$ & $\mathrm{~ns}$ & $\mathrm{~ns}$ & 12 & & \\
\hline 16 & & $\mathrm{~ns}$ & & $\mathrm{~ns}$ & 16 & & 16 & $\mathrm{~ns}$ & $\mathrm{~ns}$ & $\mathrm{~ns}$ & $\mathrm{~ns}$ & 16 & \\
\hline 23 & & & & & & 23 & 23 & $\mathrm{~ns}$ & $\mathrm{~ns}$ & $\mathrm{~ns}$ & $\mathrm{~ns}$ & $\mathrm{~ns}$ & 23 \\
\hline 37 & & & & & & & 37 & & & & & $\mathrm{~ns}$ & $\begin{array}{ll}\mathrm{ns} \\
\end{array}$ \\
\hline & & & & N1 & & & & & & VAl & $\mathrm{MP2}$ & & \\
\hline DIV & 0 & 4 & 8 & 12 & 16 & 23 & DIV & 0 & 4 & 8 & 12 & 16 & 23 \\
\hline 0 & 0 & & & & & & 0 & 0 & & & & & \\
\hline 4 & $\mathrm{~ns}$ & 4 & & & & & 4 & $\mathrm{~ns}$ & 4 & & & & \\
\hline 8 & $\mathrm{~ns}$ & $\mathrm{~ns}$ & 8 & & & & 8 & $\mathrm{~ns}$ & $\mathrm{~ns}$ & 8 & & & \\
\hline 12 & $\mathrm{~ns}$ & $\mathrm{~ns}$ & $\mathrm{~ns}$ & 12 & & & 12 & $\mathrm{~ns}$ & $\mathrm{~ns}$ & $\mathrm{~ns}$ & 12 & & \\
\hline 16 & ns & ns & $\mathrm{ns}$ & $\mathrm{ns}$ & 16 & & 16 & $\mathrm{~ns}$ & ns & $\mathrm{ns}$ & $\mathrm{ns}$ & 16 & \\
\hline 23 & & & & & & 23 & 23 & & & & $\mathrm{~ns}$ & $\mathrm{~ns}$ & 23 \\
\hline 37 & & & & & & ns & 37 & & & & & & \\
\hline b & & Post & syn & pti & & & & Leg & end & Col & or-n & nap & \\
\hline & & & & & & & & & & & & & \\
\hline DIV & 0 & 4 & 8 & 12 & 16 & 23 & & No-e & spre & ssion & & & \\
\hline 0 & $\overline{0}$ & & & & & & & & & & & & \\
\hline 4 & $\mathrm{~ns}$ & 4 & & & & & & valu & & Ger & e ex & press & sion \\
\hline 8 & $\mathrm{~ns}$ & $\mathrm{~ns}$ & 8 & & & & & 0.05 & & & $n$ & & \\
\hline 12 & $\mathrm{~ns}$ & $\mathrm{~ns}$ & $\mathrm{~ns}$ & 12 & & & & gulat & & $U$ & $p$ & Do & $\mathrm{wn}$ \\
\hline 16 & $\mathrm{~ns}$ & $\mathrm{~ns}$ & $\mathrm{~ns}$ & $\mathrm{~ns}$ & 16 & & & 0.05 & & & & & \\
\hline 23 & $\mathrm{~ns}$ & $\mathrm{~ns}$ & $\mathrm{~ns}$ & $\mathrm{~ns}$ & $\mathrm{~ns}$ & 23 & & 0.00 & & & & & \\
\hline 37 & $\mathrm{~ns}$ & & & & & $\begin{array}{ll}\mathrm{ns} \\
\end{array}$ & & 0.000 & & & & & \\
\hline
\end{tabular}

DIV on which the statistical test is done for all the multiple comparisons); non-expressing genes (grey boxes). Colour boxes for expressing genes with $P<0.05$. Upregulated (red) and downregulated (green) genes are represented with increasing three colour-scale intensities for $P \leq 0.5, P \leq$ 0.005 and $P \leq 0.0005$ respectively

GEN-Q18 cultures, the TBR $1^{+}$neuronal population tended to decrease from $7 \pm 3 \%$ at 23 DIV to $4 \pm 1 \%$ at 37 DIV (Fig. 10c). Representative images for nuclear TBR1 staining along with Map2b at 23 DIV and 37 DIV are shown in Fig. 10d for Ctr-Q33 and in Fig. S8b for GEN-Q18. Gene expression analysis in Ctr-Q33 and GEN-Q18 cultures showed an upregulation of the glutamatergic, TBR1 at 16 DIV, shown previously in Fig. 5 and Fig. S4. Additionally, differentiated hPSCs upregulated the sodium-dependent inorganic phosphate cotransporter, SLC17A6 or VGLUT1 gene from 16 DIV onwards (Fig. 10j and k, for Ctr-Q33 and GENQ18, respectively).

\section{Striatal Projection Neurons Are Primarily Observed at 37 DIV}

We specifically checked the generation of GABAergic MSNs by immunolabelling for CTIP2 (25B6) and DARPP-32 (Fig. 11 and Tables 2 and 3). Ctr-Q33 and GEN-Q18 neuronal cultures clearly exhibited DARPP- $32^{+} / \mathrm{Map}^{2} \mathrm{~b}^{+}$neurons at 37 

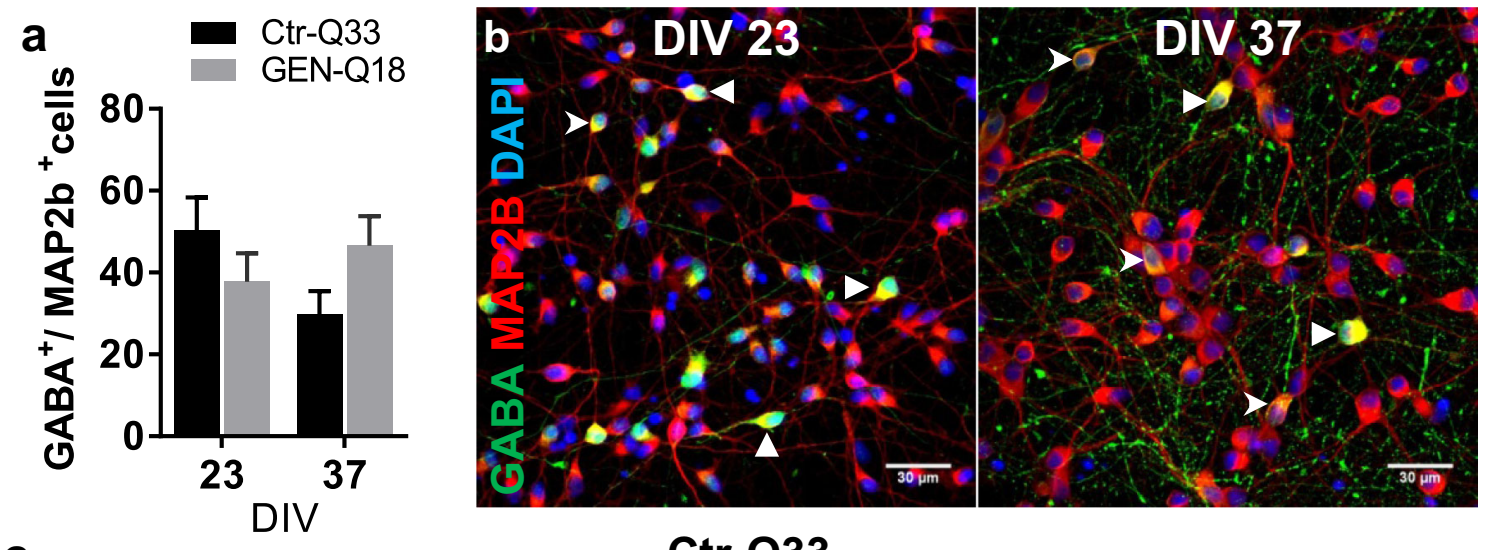

C

Ctr-Q33
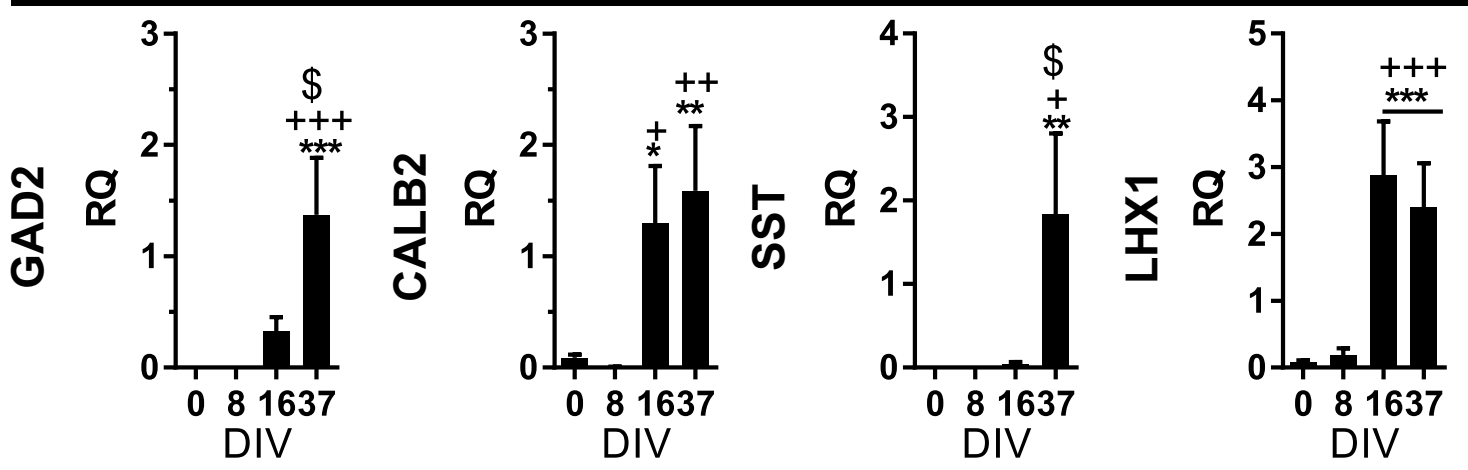

$\underline{\text { d }}$

GEN-Q18

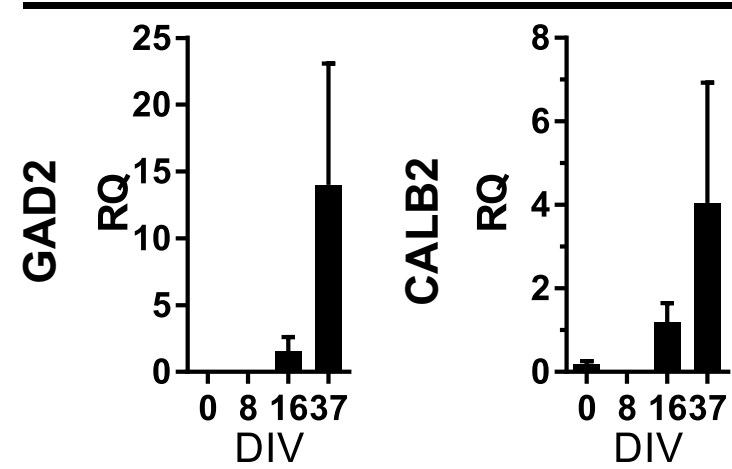

Fig. 9 Ctr-Q33 and GEN-Q18 hPSC-derived GABA+ neurons. Proportion of GABA+ cells relative to MAP2b + in Ctr-Q33 (black) and GEN-Q18 (grey) at 23 and 37 DIV (Mean \pm SEM; two-way ANOVA followed by Tukey's multiple comparison test, ns $P>0.05$ ). b Representative GABA (green) staining along with the neuronal MAP2b (red) and DAPI (blue) at both DIVs by Ctr-Q33 hiPSC-derived neuronal cultures. Triangular arrowheads point intense GABA+ neurons and spiky

DIV, and without strong differences among cell lines (6.0 \pm $0.3 \%$ and $4.0 \pm 1.4 \%$, respectively; Fig. 11a). Conversely, the differentiation of hPSC generated abundant CTIP2 $(25 \mathrm{~B} 6)^{+} /$ Map2 ${ }^{+}$neurons at 23 and 37 DIVs. Ctr-Q33 cultures contained $62 \pm 12 \%$ and $55 \pm 10 \%$ of $\mathrm{CTIP}^{+}$cells at 23 DIV and 37 DIV, respectively (Fig. 11c). GEN-Q18 neuronal cultures contained $55 \pm 14 \%$ at 23 DIV and $43 \pm 3 \%$ of $\mathrm{CTIP}^{+}$cells at 37 DIV (Fig. 11b). Both cell lines contained equivalent proportions of double-labelled DARPP- $32^{+}$and $\mathrm{CTIP}^{+}$neurons (Fig. 11c). Representative staining at both

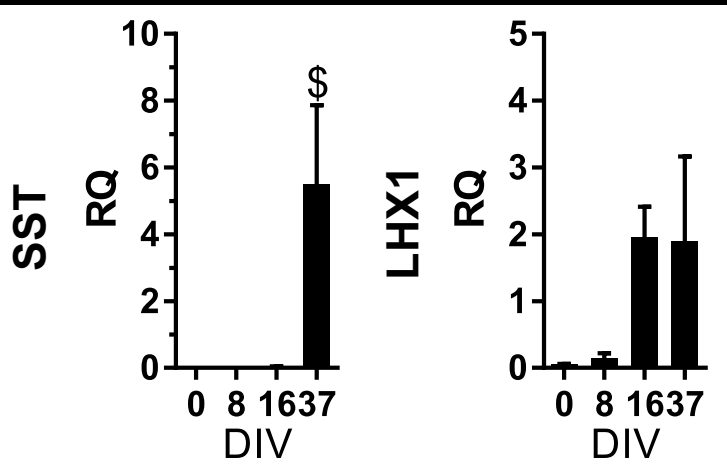

arrowheads highlight low GABA+ neurons. Scale bar $30 \mu \mathrm{m}$. Quantitative GABAergic-related gene expression profile along c CtrQ33 and d GEN-Q18 neuronal differentiation. (Mean \pm SEM; one-way ANOVA followed by Tukey's multiple comparison test, relative to 0 DIV $* P<0.05$, ** $P<0.01$, *** $P<0.001$; to 8 DIV $+P<0.05,++P<0.01$, $+++P<0.001$; to 16 DIV $\$ P<0.05, \$ \$ P<0.01, \$ \$ \$ P<0.001)$

DIVs in Ctr-Q33 hiPSC-derived neuronal cultures are shown in Fig. 11d and e and Fig. S8a and b in GEN-Q18.

Quantitative expression of striatal-related neuronal genes was observed along the neuronal differentiation (Fig. 12). Confirming previous results from our group [19, 28], two well-known MSNs markers, PPP1R1B/DARPP-32 and CALB1, were expressed throughout the differentiation with high levels at DIV 0 and DIV 37 (Fig. 12a-d). Conversely, ZNF503 (zinc finger protein 503) and BCL11B/CTIP2 genes were induced from DIV 16 onwards (Fig. 11e-h). The 
Table 2 Neuronal subtype cell counts in Ctr-Q33 hiPSC-derived neuronal cultures at 23 and 37 DIV

\begin{tabular}{|c|c|c|c|c|c|c|c|c|c|c|c|c|c|c|c|c|}
\hline \multirow{2}{*}{$\frac{\text { Ctr Q33 }}{\text { DIV }}$} & \multicolumn{2}{|c|}{ DARPP-32 } & \multicolumn{2}{|c|}{ DARPP-32/Map2b } & \multicolumn{2}{|c|}{ Ctip2 (25B6) } & \multicolumn{2}{|c|}{ DARPP-32/Ctip2 } & \multicolumn{2}{|c|}{ Map2b } & \multicolumn{2}{|c|}{ GABA/Map2b } & \multicolumn{2}{|c|}{ TH/Map2b } & \multicolumn{2}{|c|}{ TBR1/Map2b } \\
\hline & 23 & 37 & 23 & 37 & 23 & 37 & 23 & 37 & 23 & 37 & 23 & 37 & 23 & 37 & 23 & 37 \\
\hline Diff & 4 & 4 & 4 & 4 & 4 & 4 & 4 & 4 & 4 & 4 & 4 & 4 & 4 & 4 & 4 & 4 \\
\hline $\mathrm{CSs}$ & 2 & 2 & 2 & 2 & 2 & 2 & 2 & 2 & 2 & 2 & 2 & 2 & 2 & 2 & 2 & 2 \\
\hline Mean & 0.3 & 6.3 & 0.2 & 6.0 & 61.8 & 54.8 & 0.1 & 5.9 & 97.0 & 94.5 & 50.4 & 29.8 & 19.3 & 35.6 & 3.9 & 7.1 \\
\hline SD & 0.1 & 1.7 & 0.1 & 0.6 & 24.1 & 19.1 & 0.1 & 1.8 & 1.4 & 7.8 & 15.9 & 11.5 & 3.3 & 10.7 & 0.6 & 0.8 \\
\hline SEM & 0.1 & 0.8 & 0.0 & 0.3 & 12.0 & 9.5 & 0.1 & 0.9 & 0.7 & 3.9 & 8.0 & 5.7 & 1.6 & 5.4 & 0.3 & 0.4 \\
\hline$P$ value & \multicolumn{2}{|c|}{$0.0062 *$} & \multicolumn{2}{|c|}{$0.0034 * *$} & \multicolumn{2}{|l|}{0.75} & \multicolumn{2}{|c|}{$0.0072 *$} & \multicolumn{2}{|c|}{$0.0072 *$} & \multicolumn{2}{|c|}{$0.0072 *$} & \multicolumn{2}{|c|}{$0.0072 *$} & \multicolumn{2}{|c|}{$0.0072 *$} \\
\hline
\end{tabular}

neuronal differentiation promoted the expression of ADORA2A and the TAC1 (tachykinin precursor) receptors at 37 DIV (Fig. 11i-1). Other striatal-related genes, PENK (ENK), OPRM1 ( $\mu$-opioid receptor), DR1 and DR2 receptors, were detected at 37 DIV (Fig. 12m-t).

\section{Three Large Patterns of Spontaneous Neuronal Activity Are Displayed by Ctr-Q33 hiPSC-Derived Neurons at 37 DIV}

To assess whether the physiological maturation of the cultures promoted neuronal activity, we carried out a single-cell, calcium fluorescence imaging assay at 37 DIV (Fig. S9). Since sharp increases in the fluorescence calcium signal are associated with the generation of action potentials, we could examine the capacity of the hiPSC-derived neuronal cultures to spontaneously fire. We observed that both Ctr-Q33 and GEN-Q18 cultures showed spontaneous activity. For sake of clarity, however, here we show the detailed analysis of the data for the Ctr-Q33 line only.

As a first general observation, the analysis revealed a high proportion of spontaneously active regions of interests (ROIs), by $84 \%$. Since the neuronal cultures contained mostly pure Map2 $\mathrm{b}^{+}$at 37 DIV, ROIs were ascribed as neuronal somas (Fig. S9a-c). The activity profiles across neurons were asynchronous and heterogeneous (Fig. S9d), although most of the neurons exhibited bursting events, i.e. large amplitude firings that correspond to concatenated action potentials. To characterize the patterns of activity displayed by hPSC-derived neurons at DIV 37, we extracted a set of neuronal features through a customized software (NETCAL) run in MATLAB. We defined the number of spikes (NS), the inter-spike interval (ISI), the number of bursts (B), the inter-burst interval (IBI), spikes inside burst, ISI inside burst and burst length (Fig. S10).

About 12,000 hPSC-derived neurons were analysed. The different patterns of spontaneous activity were segregated by analysing the set of neuronal features through PCA and k-means algorithms (Fig. 13a). Each of the neuronal features displayed different relative contributions to the PCA axes (termed PC1 and PC2) as shown in Fig. S10. As a central result of the classification, PC1 mostly conveyed the IBIs feature, with a relative contribution of $84 \%$, while the PC2 mostly conveyed he NS feature, with a relative contribution of $79 \%$ (Fig. 13a). Thus, neurons close to the PC1 axis and distant from the origin $(0,0)$ effectively exhibited high IBI values and low NS. This characteristic ensemble of neurons was classified as "low firing neurons" (Fig. 13a). Conversely, neurons near and above the PC2 axis (Fig. 13a) elicited abundant spikes but portrayed low IBI and were classified as "high firing neurons". In between these phenotypes, there existed a group of neurons clustered at the centre of the PCA plot and that was classified as "intermediate firing neurons" (Fig. 13a). Illustrative neuronal traces for the different classifications are shown in Fig. 13b.

Table 3 Neuronal subtype cell counts in GEN-Q18 hESC-derived neuronal cultures at 23 and 37 DIV

\begin{tabular}{|c|c|c|c|c|c|c|c|c|c|c|c|c|c|c|c|c|}
\hline \multirow{2}{*}{$\begin{array}{l}\text { GEN Q18 } \\
\text { DIV }\end{array}$} & \multicolumn{2}{|c|}{ DARPP-32 } & \multicolumn{2}{|c|}{ DARPP-32/Map2b } & \multicolumn{2}{|c|}{ Ctip2 (25B6) } & \multicolumn{2}{|c|}{ DARPP-32/Ctip2 } & \multicolumn{2}{|c|}{ Map2b } & \multicolumn{2}{|c|}{ GABA/Map2b } & \multicolumn{2}{|c|}{ TH/Map2b } & \multicolumn{2}{|c|}{ TBR1/Map2b } \\
\hline & 23 & 37 & 23 & 37 & 23 & 37 & 23 & 37 & 23 & 37 & 23 & 37 & 23 & 37 & 23 & 37 \\
\hline Diff & 3 & 3 & 3 & 3 & 3 & 3 & 3 & 3 & 3 & 3 & 3 & 3 & 3 & 3 & 3 & 3 \\
\hline CSs & 2 & 2 & 2 & 2 & 2 & 2 & 2 & 2 & 2 & 2 & 2 & 2 & 2 & 2 & 2 & 2 \\
\hline Mean & 0.4 & 4.2 & 0.1 & 4.0 & 55.0 & 42.7 & 0.4 & 4.0 & 98.7 & 98.3 & 37.7 & 46.6 & 49.7 & 51.7 & 7.4 & 4.2 \\
\hline $\mathrm{SD}$ & 0.8 & 2.6 & 0.2 & 2.4 & 24.0 & 6.0 & 0.8 & 2.4 & 0.2 & 0.7 & 12.1 & 12.4 & 2.0 & 7.6 & 5.9 & 1.8 \\
\hline SEM & 0.4 & 1.5 & 0.1 & 1.4 & 13.9 & 3.5 & 0.4 & 1.4 & 0.2 & 0.5 & 7.0 & 7.2 & 1.2 & 4.4 & 3.4 & 1.0 \\
\hline$P$ value & 0.1169 & & 0.1233 & & 0.5298 & & 0.1156 & & 0.47 & & 0.1356 & & 0.72 & & 0.3127 & \\
\hline
\end{tabular}


Figure S11, together with S12, details the neuronal properties of each group, including the firing and bursting features. Neurons from groups G5 and G8 displayed greater firing activity compared with neurons of groups G1-G4. Neurons from group G5 elicited abundant spikes (mean of $320 \pm 75$ spikes per neurons), a high frequency of $0.5 \mathrm{~Hz}$ and a low ISI of $1.91 \mathrm{~s}$. Neurons from G7, G8 and G6 also showed high spontaneous activity with a mean of $182 \pm 27,112 \pm 18$ and $79 \pm 16$ spikes, respectively. Neurons included in these groups fired spikes at relative high frequency values in the range $0.1-0.3 \mathrm{~Hz}$ and, therefore, low ISI of 5-8 s (Fig. S11a-c). Conversely, neurons from G1 to G4 groups ( $60 \%$ of the neurons) elicited a low number of spikes (< 60 spikes per neurons), at low frequencies $<0.1 \mathrm{~Hz}$ and high ISI $>10$ s (Fig. S11a-c).

Practically all neurons, regardless the group, exhibited bursting features. The only exception was group G4, in which about $50 \%$ of the neurons were non-bursting. We thus concluded that differentiation of the Ctr-Q33 line procured intrinsically bursting neurons. However, bursting patterns were not uniform (Fig. S11d-h, i). Groups G5 and G7 (8\% of the population) displayed strong bursting activity with about 40-60 bursts along the recording time, which provided a typical IBI of 10-16 s (Fig. S11d and e). Neurons from groups G1 to G3, together with the bursting neurons of G4 (totalling $60 \%$ of the population), showed a weak bursting activity with a mean of only 1-7 bursts and intervals greater than $40 \mathrm{~s}$ (Fig. S11d and e). The remaining groups G6 and G7 (32\% of the population) displayed intermediate bursting values, in the range 7-17 bursts per neurons that provided IBIs in the range 27-60 s.

Concerning burst structure (Fig. S11f-h), Ctr-Q33 neurons in general fired a mean of 2 spikes per neuron within a burst, and with an interval of 500-800 ms. Groups G5 and G7 were an exception, and showed more spikes at lower intervals. These groups also displayed slightly longer bursts with a mean duration of 8.6 and 7.7 s, respectively (Fig. S11h). For statistic comparisons of each feature between groups, see Fig. S13.

Putting together this group classification with the PCA analysis outlined above, we note that the "low firing neurons" correspond to groups G1 to G4 and comprise $60 \%$ of the neurons (Fig. S13). The "intermediate firing neurons" correspond to groups G6 and G8 and comprise 32\% of neurons (Fig. S14). The "highly firings neurons" are the remaining groups G5 and G7 and comprise $8 \%$ of the neurons (Fig. S15). Thus, the differentiation of the Ctr-Q33 line not only led to spontaneously active neurons but also orchestrated a network in which different kinds of activity patterns could be observed.

\section{Ligand-Gated Receptors Are Expressed by Ctr-Q33-Derived Neurons at 37 DIV}

We performed a screening of several NT receptors by quantitative gene expression analysis along Ctr-Q33 hiPSC neuronal differentiation (Fig. 14). Regarding the excitatory Glu receptors, the three main NMDRAs subunits, GRIN1 (NR1), GRIN2A (NR2A) and GRIN2B (NR2B), and the AMPA subunit GRIA1 (GluR11) were induced by Ctr-Q33 hiPSC-derived cultures at DIV 37 (Fig. 14a). GRIN2D (NR2D) and GRIN3B (NR3B) along with other AMPA receptor subunits were expressed through the differentiation but they did not show significant changes in expression (Fig. S17a). GRIA2 subunit was found from DIV 8 onwards but the expression remained similar along all DIVs (Fig. S17b). For kainate receptors, Ctr-Q33 neurons upregulated the expression of GRIK4 (KA-1) at DIV 37 compared with 4-16 DIVs (Fig. 13a). GRIK3/4/5 gene expression was detected along differentiation, but with similar values across DIVs (Fig. S17). GRIK1 was detected from DIV 4 onwards but without changes (Fig. S17b).

Ctr-Q33 neurons upregulated the expression of GABR-A2/ B1/D and G2 genes at 37 DIV (Fig. 14b). Also, neurons started to express the GPCRs tachykinin receptor TACR1 (NK1R) at 12 DIV and afterwards it became upregulated at 23 DIV and 37 DIV (Fig. 14c). The Gly receptor subunit GLRA2 was induced at 37 DIV compared with 8 DIV and 12 DIV (Fig. 14d). Ctr-Q33 neurons expressed the excitatory serotonergic 5-HT receptors, $\mathrm{Na}^{+}-\mathrm{K}^{+}$5-HT channel HTR2A and Gq/G11-GPCRs HTR3A (Fig. 14e). The HTR2A receptor was detected from 4 DIV onwards and it was induced at 37 DIV. Conversely, HTR3A gene was expressed at the beginning of differentiation up to $12 \mathrm{DIV}$ with an upregulation peak at 8 DIV. The differentiation of Ctr-Q33 hiPSCs resulted in the downregulation of the excitatory ATP-gated P2RX2 gene at 37 DIV compared with 0 DIV (Fig. 14f).

\section{Neuronal Functional Maturation of hiPSC-Derived Neurons Is Facilitated by the Expression of $\mathrm{K}+, \mathrm{Na}+$, $\mathrm{Ca} 2+$ and $\mathrm{Cl}$-ion Channels}

We also analysed the expression of voltage-gated (VG) and ion-gated channels along Ctr-Q33 hiPSC neuronal differentiation (Fig. S16). The differentiation of Ctr-Q33 hiPSCs promoted the upregulation of the VGNC SCN2B $(\mathrm{Nav} \beta 2)$ at 37 DIV. Conversely, the SCN4A (Nav1.4) was downregulated at 8 and 12 DIVs compared with 0 DIV, and the SCN8A was downregulated at 8 DIV compared with 37 DIV (Fig. S16a).

The neuronal differentiation of Ctr-Q33 hiPSCs also downregulated the expression of VG-Cl ${ }^{-}$channels $\mathrm{CLCN} 1$ and the electroneutral $\mathrm{K}^{+} / \mathrm{Cl}^{-}$cotransporter SLC12A4/KCC1 genes (Fig. S16b and c, respectively). The neuron-specific $\mathrm{K}^{+}-\mathrm{Cl}^{-}$ cotransporter $2 \mathrm{KCC} 2$ (SLC12A5) gene was detected in CtrQ33 neurons along all DIVs but it did not experience changes on gene expression (Fig. S17).

In addition, the neuronal differentiation of Ctr-Q33 hiPSCs promoted the expression of several VG- $\mathrm{K}^{+}$channels including (1) the A-type rapid inactivating KCNA4 (Kv $\alpha 1.4)$; (2) the slowly/non-inactivated delayed rectifier KCNA1 (Kv1.1) and 
KCNH1 (Kv10.1); (3) the inward rectifiers KCNH2 (Kv11.1); and (4) the ATP-sensitive KCNJ11 (Kir6.2). All four subunits were induced at 37 DIV (Fig. S16c). Moreover, the resting membrane potential (RMP)-related KCKK2 subunit was upregulated at the progenitor's stage at 16 DIV compared with 0-12 DIV, and the KCNK5 subunit was downregulated at 16 DIV compared with 0 DIV (Fig. S16c). Concerning VGCC, Ctr-Q33 neurons displayed higher expression of $(\mathrm{N})$ CACNA1B (Cav2.2) and L-type CACNA1C (Cav1.2) genes at 23 DIV and 37 DIV compared with 0 DIV to 16 DIV. The $\beta$ subunits CACNB4 and the calcium/calmodulin-dependent protein CAMK2A genes were also induced at 37 DIV (Fig. S16e). Ctr-Q33 neurons also expressed the CAMK1G, CAMK4 and CASK genes but their levels were unaltered along differentiation (Fig. S17a).

\section{Mouse Striatal Environmental Cues Promote the Differentiation of hNPCs to Striatal Projection Neurons at 3 Months Post-Transplantation}

Finally, we investigated the differentiation potential of hPSCderived NPCs in vivo. We conducted a number of experiments in which GFP-expressing Ctr-Q33 hiPSC-derived NPCs at 16 DIV were transplanted into the neonatal mouse striatum (Fig. S19a). We evaluated the differentiation and integration of grafted human cells at short-term (1 month) and long-term (3 months) post-transplantation (PST) time points. A total of 18 neonatal mice were transplanted with one dying from complications related to surgery, while the other 17 mice reached adulthood without any significant alterations being observed (94\% survival). In the 17 surviving mice, we observed no sign of teratoma formation in any animal. At 1 month PST, CtrQ33-GFP cells showed good survival (total number of $\mathrm{HNA}^{+}$ cells, $3948 \pm 195 ; \%$ surviving cells, $26 \pm 1 \% ; n=6$ ). They were mainly located within the core of the graft and differentiated into striatal neurons, as they were labelled for MAP2, CTIP2 and DARPP-32 (Fig. 15a-c). Cell counts (Fig. S19c) revealed a high proportion of $\mathrm{MAP}^{+}$cells $(80 \pm 4 \%)$ at 1 month PST (Fig. 15d). Remarkably, $89 \pm 3 \%$ of transplanted hNPCs showed CTIP2 nuclear staining and $7 \pm 2 \%$ expressed DARPP-32 (Fig. 15e and f, respectively). By 3 months PST, the percentage of Ctr-Q33-GFP-derived neurons positive for these markers was slightly reduced, with $82 \pm 5 \%$ of CTIP2 ${ }^{+}$ cells and $5 \pm 1 \%$ of DARPP- $32^{+}$cells (Fig. 15 e and f). At this time point, the average graft volume was $91 \pm 4 \times 10^{6} \mu^{3}$ (Fig. 16a and Fig. S19b) and human cells located outside the core of the graft reflected increased migration (Fig. 16d, Fig. S19b and Fig. S20c). Most importantly, Ctr-Q33-GFP hNPCs-derived neurons were able to project long axons towards the external globus pallidus, a native striatum target (Fig. 16b and c and Fig. S20a and b). Electron microscopy analysis of these projections by means of GFP immunogold labelling revealed numerous synaptic connections between
Fig. 10 Ctr-Q33 and GEN-Q18 hPSC-derived TH+ and Tbr1+ neurons. Proportion of TH+ cells relative to MAP2b + in Ctr-Q33 (black) and GEN-Q18 (grey) at 23 and 37 DIV and b representative TH (green) staining along with MAP2b (red) and DAPI (blue) at both DIVs by CtrQ33 hiPSC-derived neuronal cultures. Triangular arrowheads point intense $\mathrm{TH}+$ neurons and spiky arrowheads highlight low $\mathrm{TH}+$ neurons. Scale bar $30 \mu \mathrm{m}$. c Proportion of Tbr1+ cells relative to MAP2b + in CtrQ33 (black) and GEN-Q18 (grey) at 23 and 37 DIV and d representative Tbr1 (green) staining along with MAP2b (red) and DAPI (blue) at both DIVs by Ctr-Q33 hiPSC-derived neuronal cultures. Triangular arrowheads point intense $\mathrm{Tbr} 1+$ neurons and spiky arrowheads point low Tbr $1+$ neurons. Scale bar $30 \mu \mathrm{m}$. (Mean \pm SEM; two-way ANOVA followed by Tukey's multiple comparison test, ns $P>0.05$ ). Quantitative DAergic- and glutamatergic-related gene expression profile along (e-g and i) Ctr-Q33 and (h and $\mathbf{j}$ ) GEN-Q18 neuronal differentiation. (Mean \pm SEM; one-way ANOVA followed by Tukey's multiple comparison test, relative to $0 \mathrm{DIV} * P<0.05$, ** $P<0.01$, $* * * P<0.001$; to $8 \mathrm{DIV}+P<0.05,++P<0.01,+++P<0.001$; to 16 DIV $\$ P<0.05, \$ \$ P<0.01, \$ \$ \$ P<0.001)$

host and grafted neurons, indicating that functional integration of the transplanted cells had occurred (Fig. 16e-g). Human neurons established symmetric (inhibitory) synapses (Fig. 16e) and received asymmetric (excitatory) synaptic inputs (Fig. 16f and g) from host cells, mimicking MSN circuitry within the basal ganglia. It is of note that of the population of $\mathrm{HNA}^{+}$cells found in the mouse striatum, $76 \pm 5 \%$ maintained GFP expression at 3 months PST.

\section{Robustness of the Protocol}

At the time of revision of this manuscript, this neuronal differentiation protocol has been performed a total of 399 times, 165 times with hESC lines $(41 \%)$ and 234 times with hiPSC lines $(59 \%)$. The protocol has been conducted by different staff members of our centre, including $\mathrm{PhD}$ students, technicians and post-doctoral researchers. Although differentiation was not deeply characterized on all occasions as described here, on 349 occasions (87\%), a mature neuronal phenotype was achieved, while failure only occurred on 50 occasions $(13 \%)$. These results demonstrate the robustness of the protocol.

\section{Discussion}

The neurogenesis of hPSCs is a complex process comprised by several phases and signals. This includes the neural fate specification of hPSCs, the proliferation and specification of NPCs to region-specific progenitors and their terminal differentiation to subtype-specific neurons. The neuronal differentiation is accompanied by neurites and axon outgrowth, synapse formation and maturation that finally shape the neuronal circuit. Here we modelled 

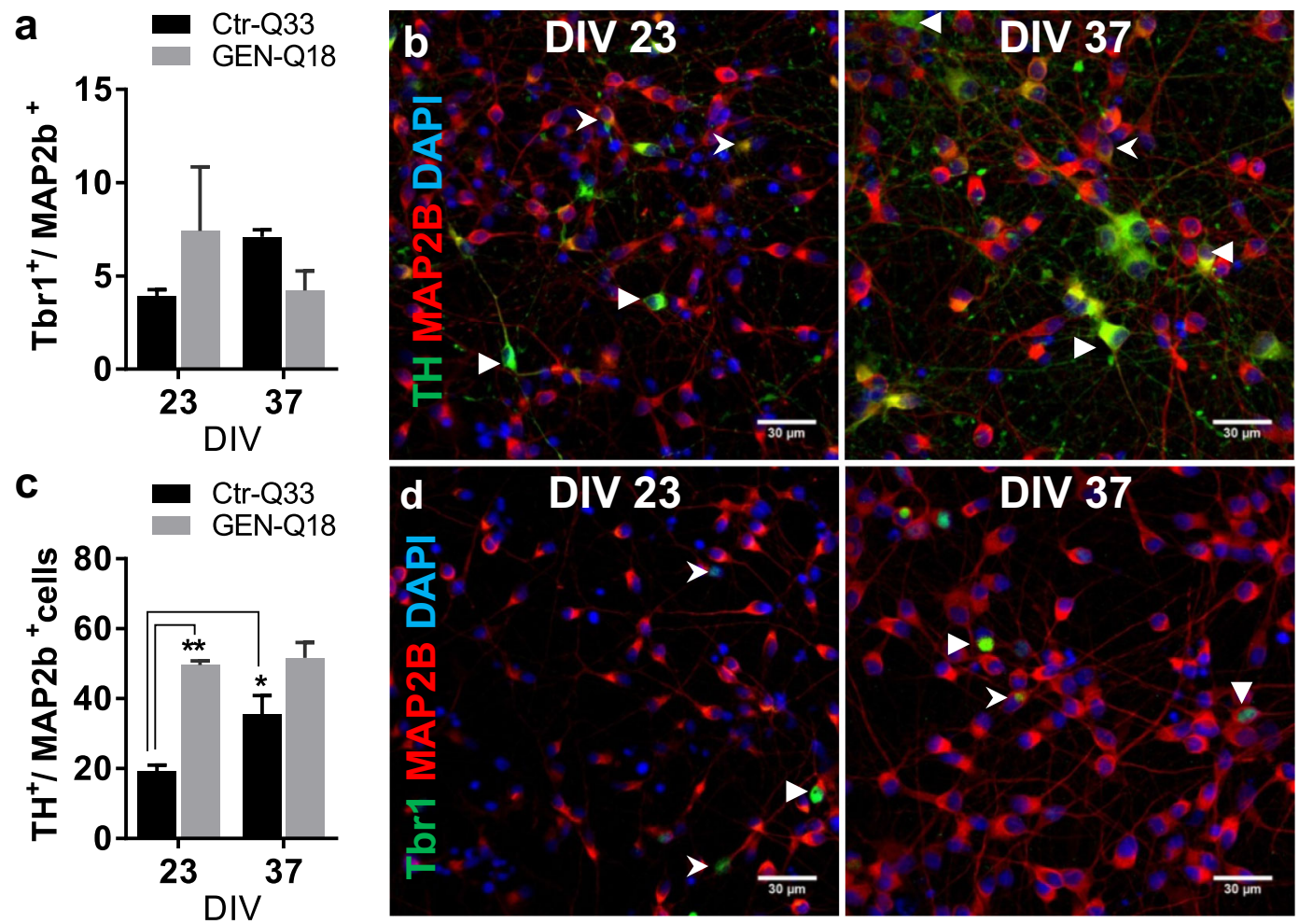

Ctr-Q33
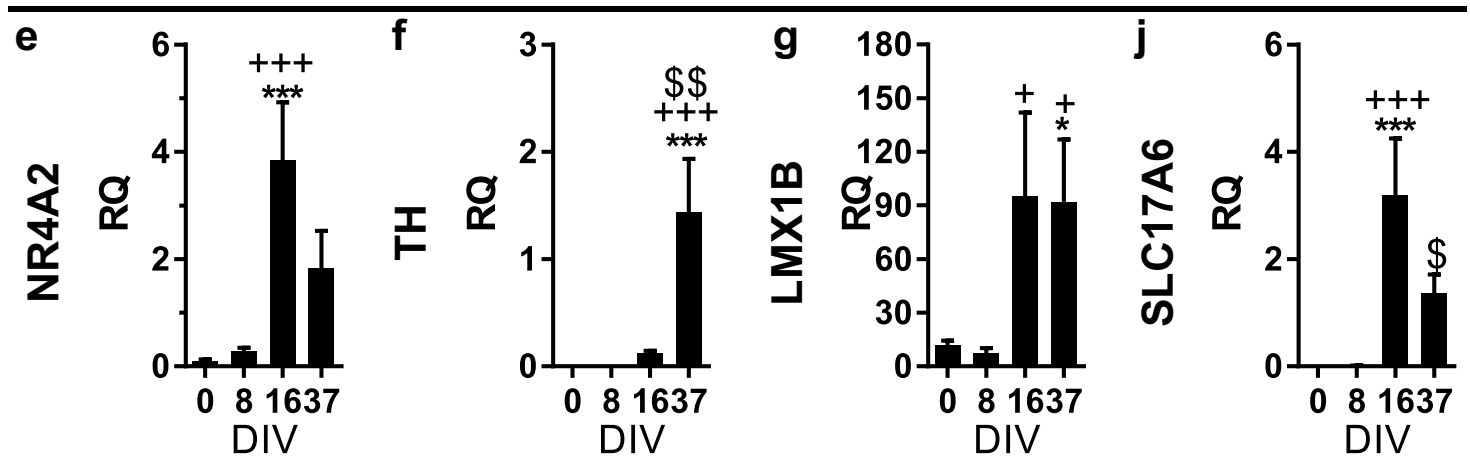

GEN-Q18
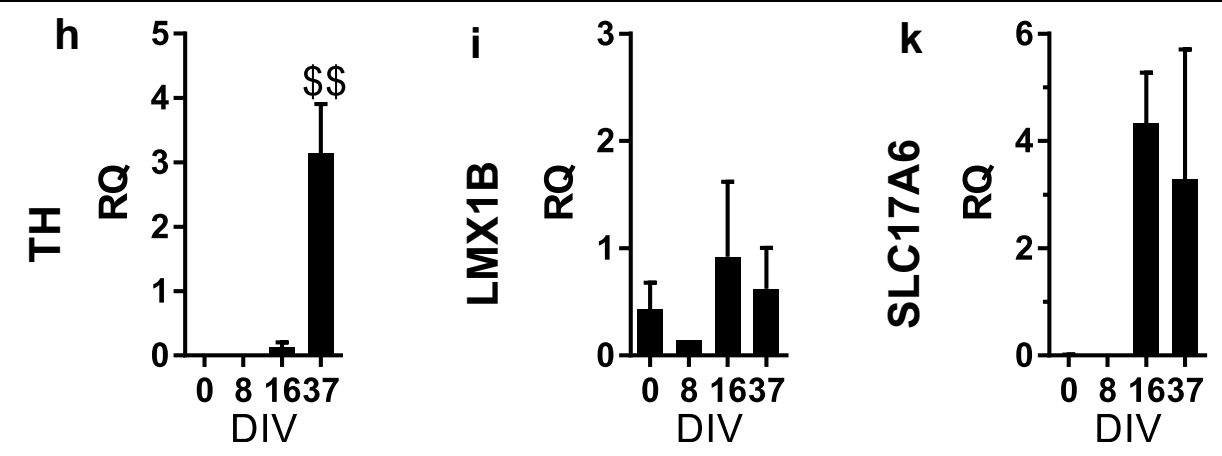

the in vitro differentiation of two hPSC lines from pluripotency to forebrain mature neurons. The neuronal differentiation generated enriched-ventral telencephalic neuronal progenitors which showed a successful in vitro functional maturation and in vivo integration.

\section{Patterning of hPSCs to Telencephalic Neural} Progenitors

The initial stages of the differentiation protocol rapidly and efficiently induced a neuroectodermal identity and by 8 DIV 

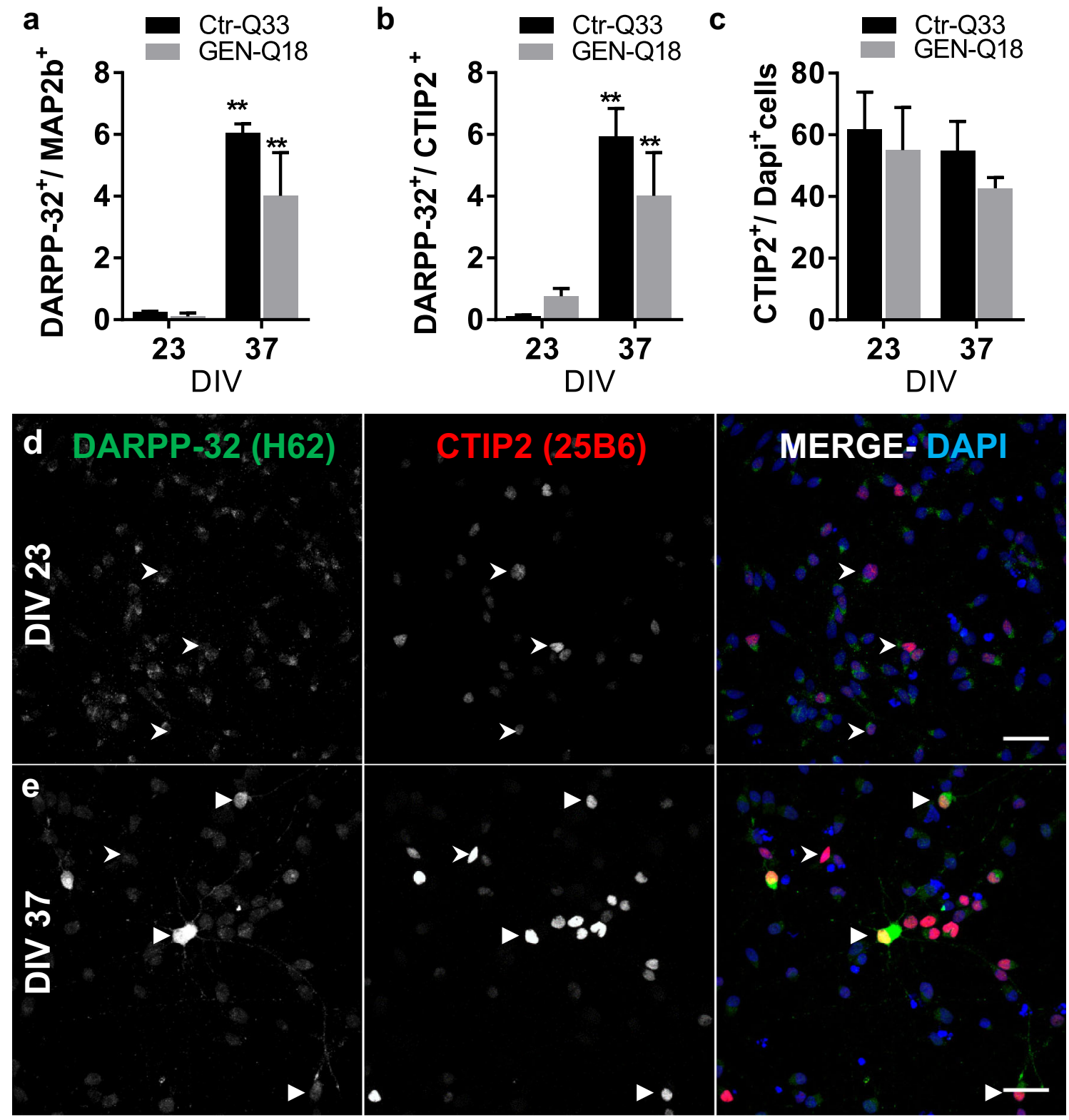

Fig. 11 Quantification of a DARPP-32+/ MAP2b+, b CTIP2+ and c double DARPP-32+/CTIP2+ in Ctr-Q33 (black) and GEN-Q18 (grey) hPSC-derived cultures at 23 and 37 DIV (Mean \pm SEM; two-way ANOVA followed by Tukey's multiple comparison test, $* P<0.05$,
$* * P<0.05$ ). Representative images for DARPP-32 (green) and CTIP2 (red) at $\mathbf{d} 23$ DIV and e 37 DIV (Arrowhead points out double-labelled neurons and arrow single-labelled-CTIP2 nucleus). Scale bar $30 \mu \mathrm{m}$ neuroepithelial rosettes, the in vitro two-dimensional equivalent of the neural tube had formed. This is faster than with other hPSC neural differentiation protocols where 10-14 days are required for rosettes to form from hPSCs. For example, Delli Carri and colleagues showed neural rosettes 15 days after induction [29], and Aubry and colleagues after 21-23 DIV [30].

The neuroepithelial cells were successfully specified to a forebrain NPC identity and not a more posterior identity as shown by expression of the forebrain marker FOXG1 and the absence of expression of midbrain, hindbrain and spinal cord markers. A ventral forebrain NPC fate was then acquired by the majority of cells based upon the expression of the Dlx family of transcription factors and the transcription factor EBF1 which play key roles in the specification of distinct populations of ventral telencephalic progenitors. This ventral specification was achieved solely by inhibiting dorsal BMP and Wnt signals $[31,32]$. The ventralising morphogen $\mathrm{SHH}$ was omitted in an attempt to avoid the specification of ventral MGE-interneuron progenitors at the expense of striatal neuron progenitors [33-35]. FOXG1 expression may have contributed to the observed efficient ventral NPC specification as it is known to inhibit canonical Wnt signalling which specifies a dorsal fate. A small population of Pax6-expressing NPCs was also observed which may 


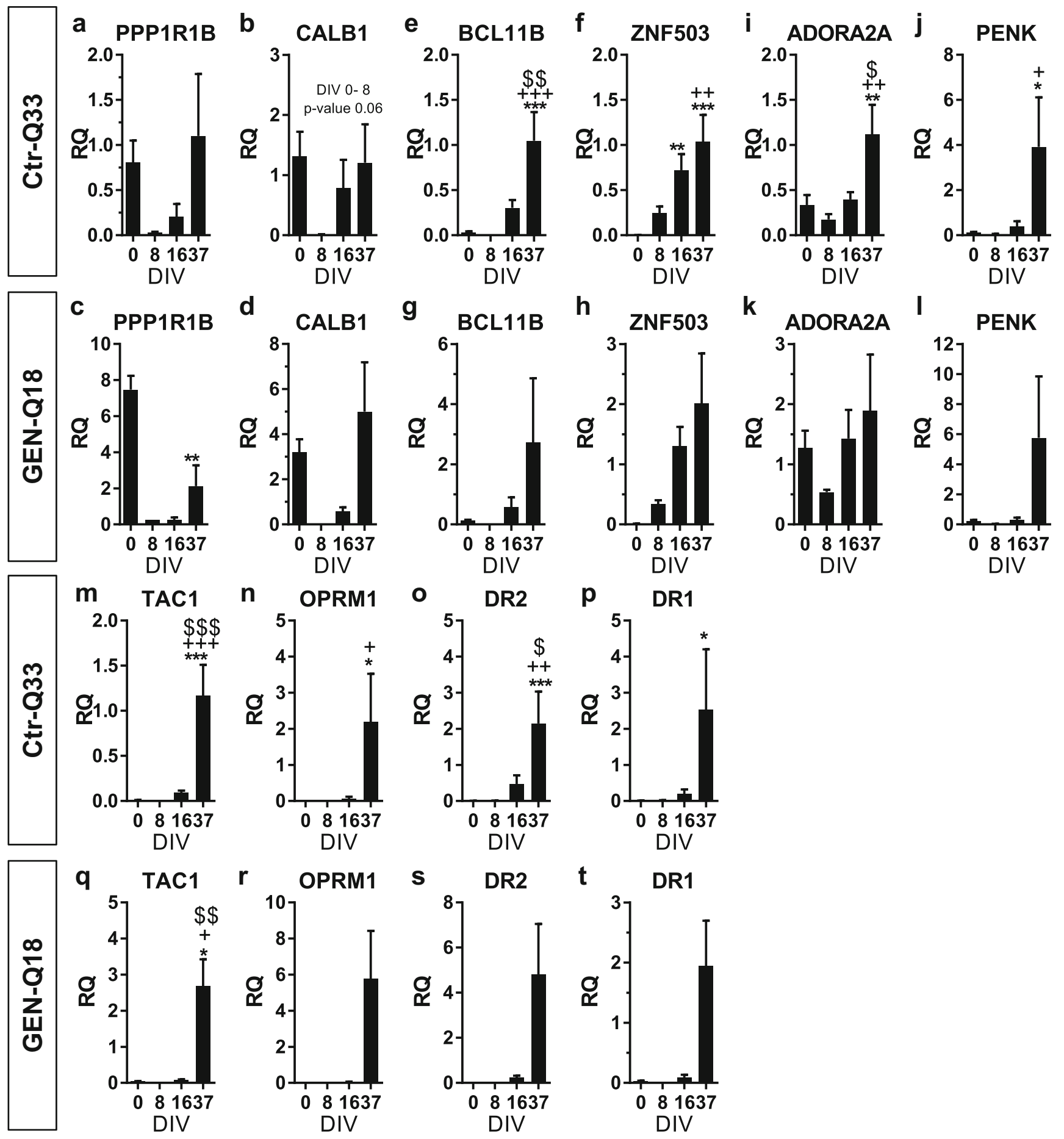

Fig. 12 Gene expression profile of striatal MSN-related genes by CtrQ33 hiPSC and GEN-Q18. a-d PPP1R1B/DARPP-32 and CALB1, e-h BCL11B/CTIP2 and ZNF503, $\mathbf{i}-\mathbf{l}$ ADORA2A and PENK and $\mathbf{m}-\mathbf{t}$ TAC, OPRM1 and dopamine receptors DR1 and DR2 along. Mean \pm SEM;

one-way ANOVA followed by Tukey's multiple comparison test, relative to $0 \mathrm{DIV} * P<0.05$, $* * P<0.01$, $* * * P<0.001$; to $8 \mathrm{DIV}+P<$ $0.05,++P<0.01,+++P<0.001 ;$ to 16 DIV $\$ P<0.05, \$ \$ P<0.01, \$ \$ \$$ $P<0.001$

be suggestive of a dorsal forebrain identity [36]. Increasing slightly the concentration of the BMP and Wnt signalling inhibitors could avoid the formation of these dorsal NPCs and potentially produce a pure population of ventral NPCs. However, Pax6 is also expressed in neuroepithelial progenitors [27]. Given that these Pax6-expressing cells also expressed ventral markers and that a low number of Tbr1-expressing neurons were observed, this suggests that most of the $\mathrm{Pax}^{+}$cells are neuroepithelial progenitors. 
Fig. 13 Segregation of Ctr-Q33 hiPSC-derived neurons by its spontaneous activity at 37 DIV. a PCA plot from active neuronal population segregated based on the neuronal features and clustered in 8 groups (differentially coloured). b Individual fluorescence trace $\left(\Delta F / F_{0}\right)(t)$ from neurons $1-3$ from a PCA with different activity patterns. $\mathbf{c}$ Pie plot of the fraction of neurons in each group (G1G8)

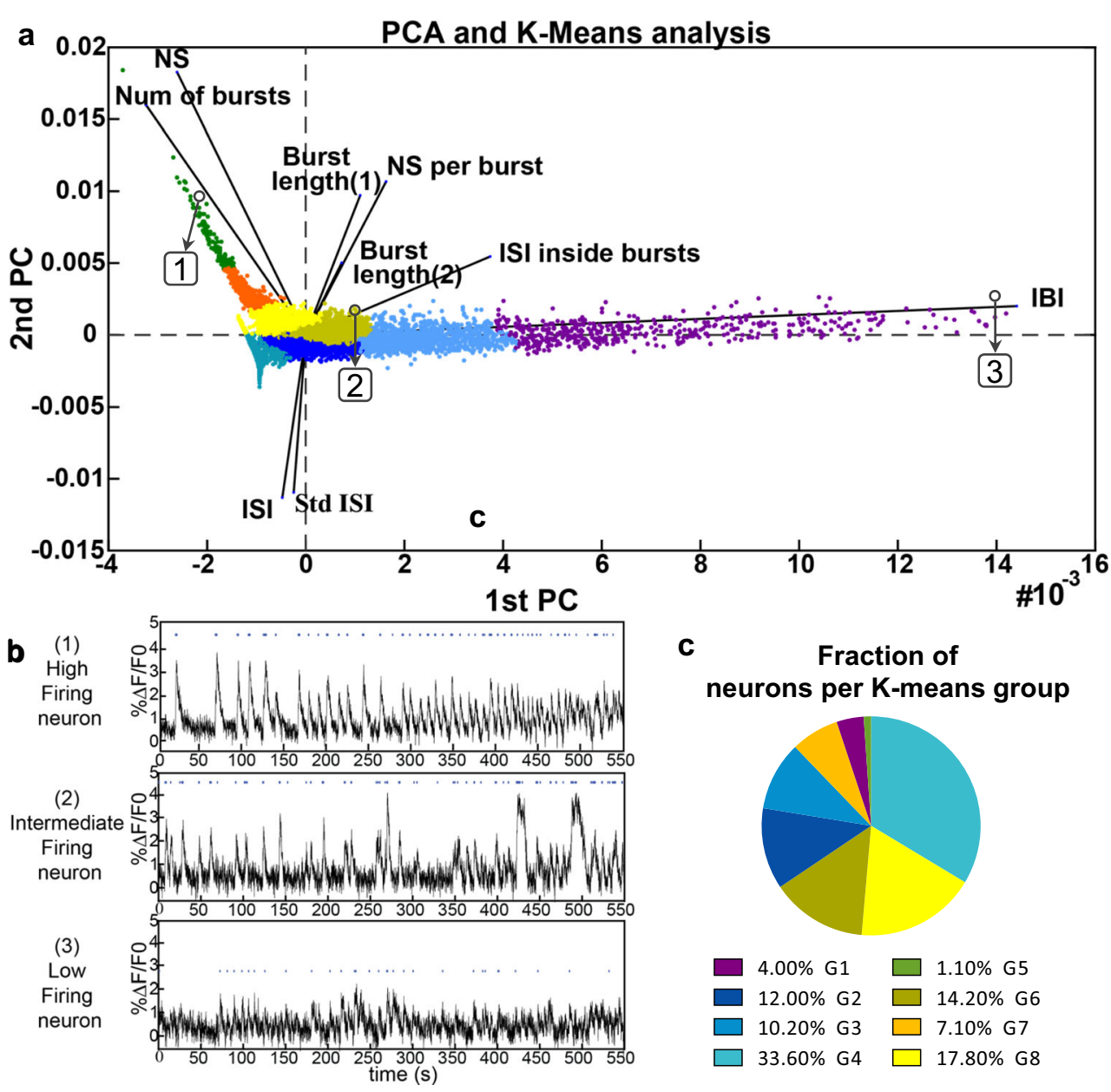

The DLX family of transcription factors and EBF1 are involved in the specification of distinct populations of ventral NPCs and neuroblasts. The DLX family is involved in specifying MSNs of the indirect pathway MSNs while EBF1 plays a role in specifying direct pathway MSNs [37-39]. At 16 DIV, more DLX-expressing NPCs were observed than EBF1expressing NPCs and this difference may be attributable to the different expression time windows of these transcription factors during striatal neurogenesis. Whereas DLX-1 and 2 are expressed by proliferative NPCs in the VZ and SVZ, EBF1 is expressed later by NPCs as they differentiate to postmitotic neuroblasts in the MZ [40-42]. Thus, the in vitro expression patterns of transcription factors involved in striatal development mirror the patterns observed in vivo indicating that the differentiation protocol recapitulates key aspects of neurodevelopment.

\section{Specification of hPSC-Derived NPCs to Postmitotic Telencephalic Neurons}

The main affected area of the brain in HD is the striatum due to the degeneration of the GABAergic MSNs. A cell therapy approach to replace the degenerated MSNs is a viable strategy to treat $\mathrm{HD}$ and for this reason, we have developed the ventral forebrain neuron differentiation protocol described here in addition to using it as a platform to study neurodevelopment in both healthy and disease contexts.

This protocol efficiently generates neurons with almost all cells acquiring a neuronal identity by the end of the protocol. This compares favourably to other striatal neuron differentiation neurons where a mixture of cell types including neurons is often obtained $[29,30,43]$. These neurons express a wide range of ion channels, NMDA receptors and synaptic-associated genes indicating that they are functional at both the individual neuron and network level. Furthermore, these neurons display a large amount of sprouting and arborisation, as well as containing spine-like structures as has been shown previously [18]. This protocol produces neurons from hPSCs in just 37 days which is faster than other striatal differentiation protocols where longer periods of time are required (see Fig. 18). The protocol developed by Arber and colleagues [35] requires a similar period of time to acquire a neuronal phenotype (37-43 DIV). However, whereas Arber et al. demonstrate firing in a low number of neurons after 93 DIV [35], here we showed activity in $84 \%$ of the analysed neurons at 37 DIV. 


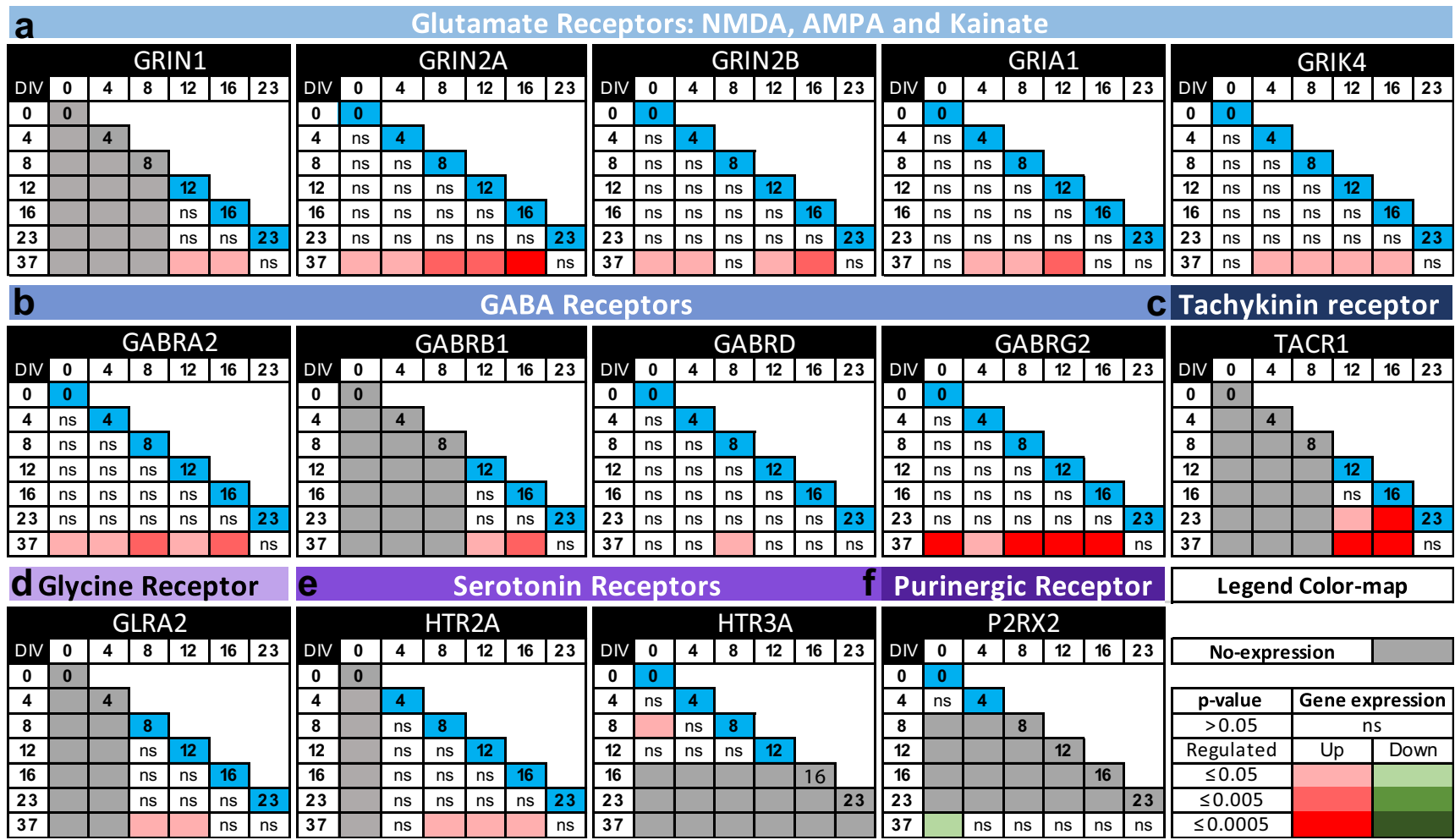

Fig. 14 Quantitative gene expression matrices of ligand ion-gated receptors expression by $\mathrm{Ctr}-\mathrm{Q} 33$-derived cultures along the neuronal differentiation (DIV vs. DIV). a Glu, b GABA, $\mathbf{c}$ tachykinin, $\mathbf{d}$ glycine, e serotoninergic and $\mathbf{f}$ purinergic receptors along Ctr-Q33 hiPSC neuronal differentiation. One-way ANOVA followed by Tukey's multiple comparison test was performed between DIVs (0 to 37). Coloured

MSNs are commonly defined by the co-expression of CTIP2 and DARPP-32 [29, 44]. Although a high number of CTIP2-expressing neurons were observed with this protocol, the number of neurons co-expressing the two proteins was $6 \%$. Published MSN differentiation protocols vary both in their reproducibility and in the amount of MSNs that they produce, ranging from 4 to $60 \%$ (see Fig. S18 and references therein). Factors that may contribute to this variability include the PSC lines that are used, how those PSC lines are maintained prior to differentiation and differences between the protocols including how and when signalling pathway activity is modified. Other sources of variability include the antibodies that are used to detect DARPP-32, their sensitivity and also the isoforms of DARPP-32 that these antibodies detect. No consensus exists on which DARPP-32 antibody is the best for detecting MSNs in vitro and as such different groups use different antibodies which recognise different epitopes [28]. Different human DARPP-32 isoforms are known to exist with the isoforms that are expressed varying according to the neurodevelopmental stage. Furthermore, DARPP-32 has the potential to undergo several post-translational modifications (PTMs) depending on the state of the cell and the signals which are being received [28] which further complicates antibody-mediated detection. Taking these factors together matrices represent all possible comparisons on the diagonal (blue box; DIV on which the statistical test is done for all the multiple comparisons); non-expressing genes are represented as grey boxes. Colour boxes are expressing genes with $P<0.05$. Upregulated and downregulated genes are represented by red and green respectively with increasing three colour-scale intensities for $P \leq 0.5, P \leq 0.005$ and $P \leq 0.0005$ respectively

illustrates the difficulties associated with using DARPP-32 as a robust marker for in vitro differentiated MSNs.

Given the large number of cells that express either CTIP2 or GABA, and the doubts associated with using DARPP-32 as MSN marker, we are of the opinion that there are more than $6 \%$ MSNs in the cultures. We propose that these additional MSNs either express isoforms or contain PTMs that are not detectable with the DARPP-32 antibody used here. Alternatively, DARPP-32 may be expressed at a low level that is below the detection threshold of the antibody. The hypothesis that there are more MSNs present in the cultures than are detected by the antibody is strengthened by the observed expression of several other genes that label MSNs at the later stages of the differentiation protocol.

A small percentage of glutamatergic cortical neurons was detected based on the expression of known cortical markers. This low abundance correlates with the small amount of Pax6expressing dorsal NPCs that was observed at an earlier stage of the differentiation. We also consider the possibility that a fraction of the neurons expressing CTIP2 but not DARPP-32 is also cortical neurons as in addition to being expressed in the striatum, it is also expressed in layer 5 of the cortex $[45,46]$.

An abundant amount of tyrosine hydroxylase (TH)-expressing neurons was also detected. Although TH is a marker 


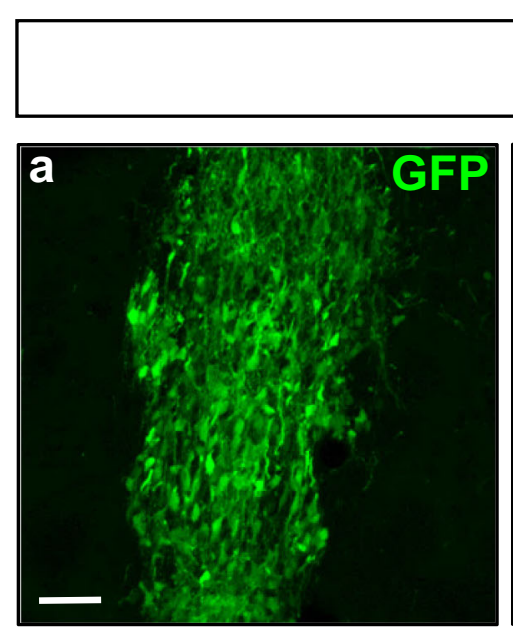

\section{1 month PST}
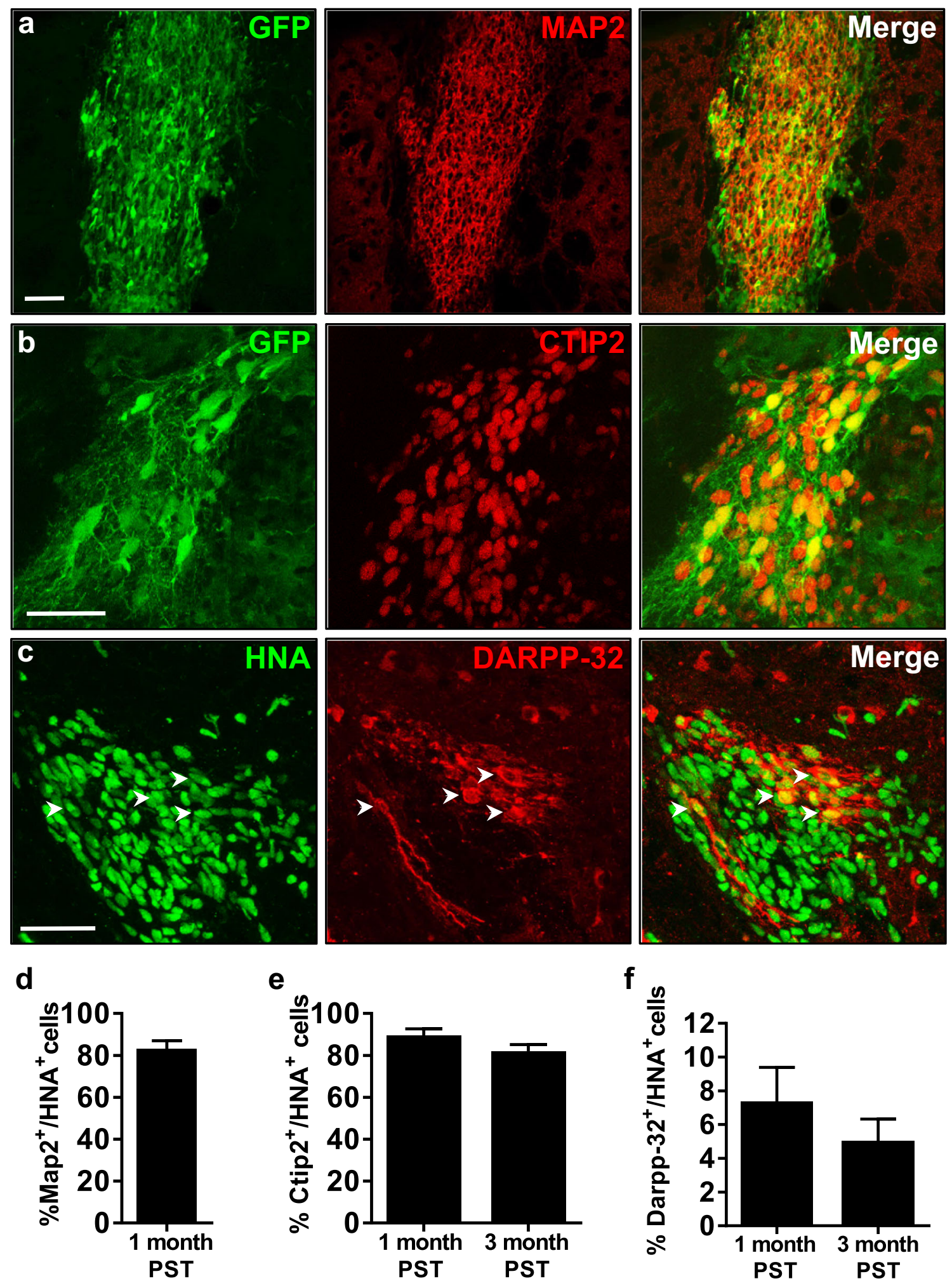

Fig. 15 hNPCs transplanted into the mouse neonatal striatum differentiate into striatal neurons. Pictures of Ctr-Q33-GFP hiPSCderived neurons at 1 month PST labelled with GFP or HNA (green)

and a MAP2, b Ctip2 or $\mathbf{c}$ DARPP-32 (red). Cell counts for MAP2 d at 1 month PST and CTIP2 $\mathbf{e}$ and DARPP-32 f at 1 and 3 months PST. Scale bars $50 \mu \mathrm{m}$ 


\section{3 months PST}
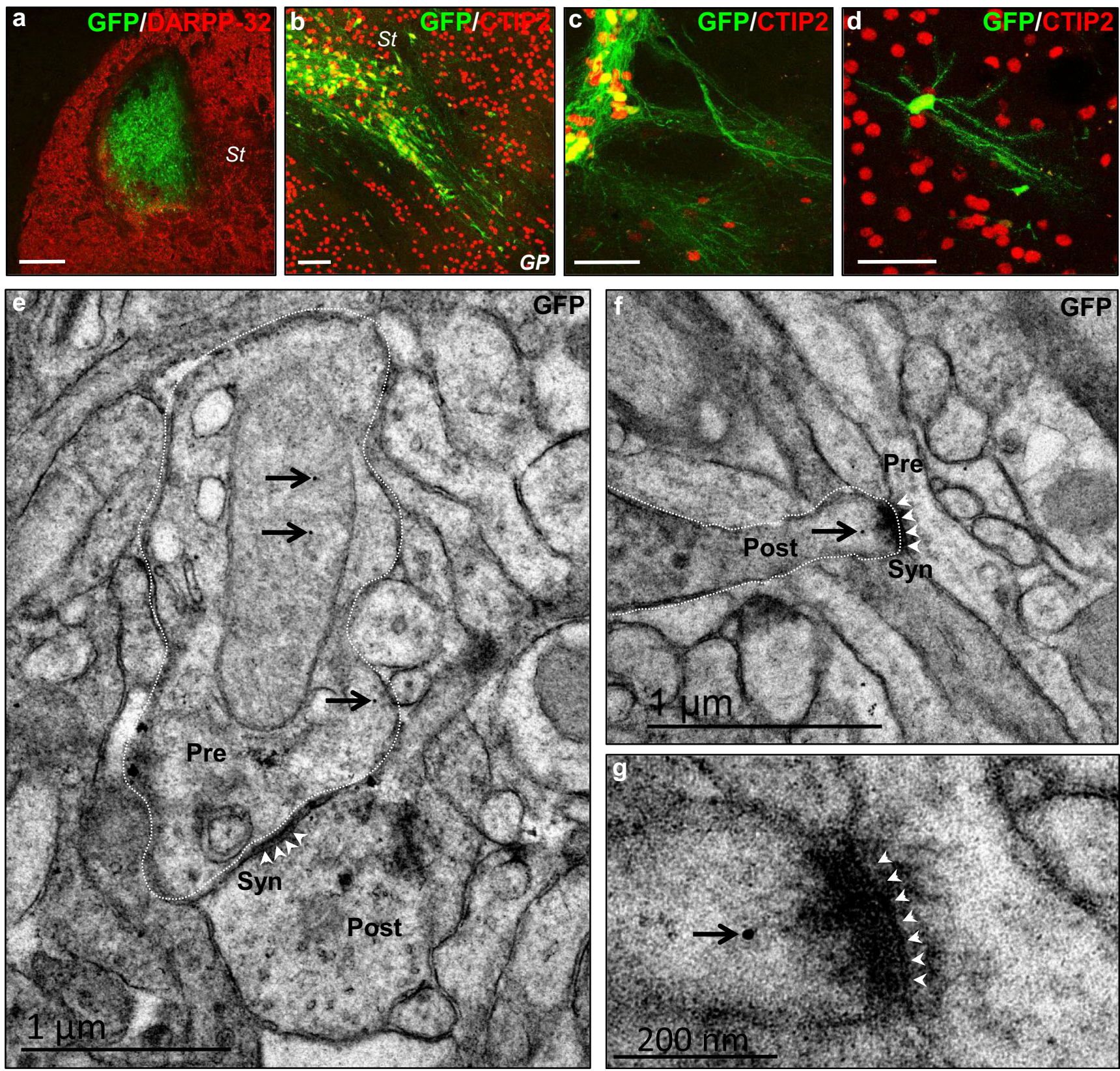

Fig. 16 Transplanted hNPC-derived neurons project towards MSN targets and establish synaptic connections at 3 months posttransplantation. a Low-magnification picture of a coronal section of the mouse striatum showing grafted Ctr-Q33-GFP human cells at 3 months PST. b-d Sagittal sections of the mouse striatum showing CTIP2 ${ }^{+}$human cells extending $\mathrm{GFP}^{+}$projections towards the external globus pallidus area. e-g Ultra-thin sections at the mouse striatum-globus pallidus level showing TEM images of human neurites (dotted lines), identified by

of midbrain dopaminergic neurons, in this case, it is unlikely that these neurons have this identity as during differentiation, we did not detect expression of Pax2 which is necessary for midbrain neurogenesis [47]. Furthermore, midbrain dopaminergic neuron differentiation requires SHH, FGF8 and $\mathrm{Wnt} / \beta$ - means of GFP immunogold labelling (black arrows). e Human neurite (pre) establishing a symmetric inhibitory synapse (white arrowheads) with a host mouse cell (post). f Human neurite (post) receiving an asymmetric excitatory synapse (white arrowheads) from a host mouse cell (pre). g High magnification of the postsynaptic density of the synapse (white arrowheads) illustrated in f. St striatum; GP globus pallidus; Pre presynaptic; Post postsynaptic; Syn synapse. Scale bars $200 \mu \mathrm{m}$ in $\mathbf{a} ; 50 \mu \mathrm{m}$ in $\mathbf{b}, \mathbf{c}, \mathbf{d}$

catenin signalling which are either inhibited or absent in this protocol [48]. These TH-expressing neurons are likely to be a subpopulation of striatal interneurons as during neurodevelopment, these interneurons are generated by the medial ganglionic eminence $[49,50]$ which is adjacent to the 
lateral ganglionic eminence that generates the MSNs. Gradients of the same signalling molecules are involved in generating these regions, and many of the same transcription factors are involved in the development of both of these regions [51]. From an in vitro perspective, slight variations during the patterning stage of the differentiation protocol in the concentrations of the small molecules received by individual cells and the processing of these signals could drive them to become MGE NPCs instead of LGE NPCs. The striatal interneuron identity hypothesis was confirmed when we observed co-expression of TH and GABA as has been described previously for a subpopulation of striatal interneurons [52]. This GABAergic striatal interneuron identity was further supported by the observed expression of various interneuron-related genes at the transcriptomic level at the same differentiation stage.

\section{Synaptic and Functional Maturation of hPSC-Derived Telencephalic Neurons}

Effective in vitro neuronal differentiation protocols require the production of functionally mature neurons where gene expression profiles and functional properties mirror those of the corresponding endogenous neuronal types. Functional maturation requires the expression of a range of ion channels, receptors, intracellular signalling components and synaptic function-related genes whose expression levels may alter as maturation proceeds. The expression of ion channels, receptors and intracellular signalling components contributes to the intrinsic functional properties of individual neurons while functional neuronal network development relies upon expression of the synaptic machinery.

The expression of ligand-gated channels and VGCs contributes to the acquisition of passive and active neuronal properties, including excitability and the ability to elicit and transmit action potentials [53]. We observed upregulation of the RMP-related channel subunit, which is likely to be at least partly responsible for the negative resting membrane potential of hPSC-derived neurons that we have reported previously [18]. Furthermore, expression of voltage-gated sodium channels (VGNCs) was also observed which correlates with the ability of hPSC-derived neurons to display VGNC currents [23]. At the later stages of neuronal differentiation, we observed expression of several voltage-gated potassium channels (VGKCs) and voltage-gated calcium channels (VGCCs) that are responsible for driving functional maturation. Previous work showed that the blockage of VGCCs results in a dramatic suppression of action potentials by hPSCderived neurons and a decline in the RMP [45]. Taken together, the expression of these ion channel-related genes contributes to neuronal electrophysiological maturation and indicates that this protocol produces functionally maturing neurons.

Neuronal differentiation and maturation also require intracellular calcium transients $\left(\mathrm{iCa}^{2+}\right)$ and calcium-mediated signalling events. $\mathrm{iCa}^{2+}$ can arise from two main sources, from extracellular $\left(\mathrm{eCa}^{2+}\right)$ influx which is triggered by growth factors or NT-mediated depolarization, or from the release of $\mathrm{iCa}^{2+}$ stores [54]. Increased $\mathrm{iCa}^{2+}$ concentration initiates downstream signalling cascades that result in modulation of gene expression [55], receptor trafficking [56] and synapse remodelling [55], among other functions. Thus, it is essential that in vitro differentiated neurons develop a functional calcium sensing and signalling system.

In most $\mathrm{CNS}$ neurons, $\mathrm{Ca}^{2+}$ influx is mediated by the opening of surface L-Type VGCCs, and also AMPA and NMDA receptors. The upregulation of such genes was observed at the later stages of our differentiation protocol. Other VGCCs, specifically the $\mathrm{N}$ - and $\mathrm{P} / \mathrm{Q}$-types, are highly expressed at the presynapse where they mediate $\mathrm{Ca}^{2+}$-dependent presynaptic NT-vesicle release to the synaptic cleft via direct interaction with SNAREs and vesicle-adapter proteins $[57,58]$. In response to an incoming action potential, presynaptic VGCCs open and allow $\mathrm{iCa}^{2+}$ levels at the terminal buttons to rise from basal nanomolar concentrations to over $50 \mu \mathrm{M}$ [59]. Expression of N-type VGCCs and several SNARE proteins was observed in our neuronal cultures suggesting that these neurons have functional $\mathrm{Ca}^{2+}$-mediated signalling pathways and are developing functional synapses. Further evidence of $\mathrm{Ca}^{2+}$-mediated neuronal maturation was inferred from the observed expression of the auxiliary $\beta 4$-L-type VGCC. This subunit modulates the surface expression and gating of the $\alpha 1$-VGCC subunit, and promotes neuronal maturation by repressing the action of the heterochromatin protein 1 gamma (HP1 $\gamma)$ [60, 61]. $\beta 4-V G C C$ subunit expression at the later stages of the differentiation protocol might mediate $\mathrm{Ca}^{2+}$-dependent HP1 $\gamma$ repression and facilitate neuronal maturation. Taken together, we conclude that these neurons have functional $\mathrm{Ca}^{2+}$ signalling and homeostatic systems that have the potential to produce $\mathrm{iCa}^{2+}$ transients which are essential for neuronal maturation.

Synaptic maturation requires the expression of presynapticrelated proteins, including vesicle-associated proteins, and postsynaptic-related proteins, such as NMDARs and AMPA subunits. Such gene expression patterns were observed at the later stages of the differentiation protocol, indicating that synapse formation and maturation were occurring in our neuronal cultures which in turn would result in the formation of neuronal networks. Previously we showed that hPSC-derived neurons responded to excitatory (Glu) and inhibitory (GABA) NTs and that they evoked miniature postsynaptic excitatory and inhibitory currents in respond to Glu and GABA, respectively [18]. In addition, we showed that the hPSC-derived neurons were sufficiently mature to perform the GABA switch, where GABA evokes miniature inhibitory currents instead of excitatory currents which is characteristic of immature neurons [18]. This prior work complements the observations of synaptic maturation described here. In the present 
work, however, dynamic expression of the genes responsible for the GABA switch was not detected. Neither upregulation of the neuron-specific cotransporter $\mathrm{KCC} 2$ nor downregulation of the immature neuron-specific NKCC1 cotransporter, which is responsible for the reduction of intracellular $\mathrm{iCl}^{-}$ ions, was observed [62-64]. This discrepancy may be due to the fact that in this work, gene expression was studied at the neuronal population level where different neuronal types at different maturation stages are present. A population level analysis may mask the changes of gene expression associated with the GABA switch if it only occurred in a subset of the total population.

To validate the apparent functionally mature state of the neurons indicated by the gene expression studies, we performed high-speed somatic $\mathrm{Ca}^{2+}$ imaging at the endpoint of the protocol. We found that a high proportion of neurons displayed spontaneous $\mathrm{iCa}^{2+}$ transients. These transients were characterized by a fast $\mathrm{Ca}^{2+}$ rise followed by a slow decay, a hallmark of elicited action potentials [65]. Neuronal activity typically appeared in the form of bursts, which corresponds to trains of action potentials, although the degree of activity varied between neurons. Analysis of the spontaneous firing neuronal activity identified three main groups which varied according to their activity and were termed "high firing" (HF), "intermediate firing" (IF) and "low firing" (LF) neurons. HFNs comprised $8 \%$ of the neurons which fired strong bursting events every $10 \mathrm{~s}$. In contrast, LFNs comprised $60 \%$ of the neurons, exhibited weak bursting every $40 \mathrm{~s}$ or more, and in general tended to elicit single spike events at low rate. The IF neurons, which comprise the remaining $32 \%$, displayed an intermediate behaviour firing between $10 \mathrm{~s}$ and $40 \mathrm{~s}$. LFNs are likely to be functionally immature neurons as they very rarely generated bursts of spikes which is a feature of more mature neurons.

Various reasons are likely to contribute to the variety of neuronal activity patterns observed here. As described above, different neuronal types are present within the same culture and each neuronal type is likely to have a different activity pattern and maturation rate. Furthermore, the rate of neuronal maturation is unlikely to be uniform, and thus it is probable that neurons of the same type are at different maturation stages both at the individual cell and network level which would contribute to the heterogeneous activity state. Also, given that both glutamatergic and GABAergic neurons are present in the same culture, similar cells within a culture may experience different local conditions in terms of excitatory and inhibitory inputs which would further contribute to variable activity. This protocol produces neurons in a highly efficient manner, but the absence of astrocytes could also contribute to the abundance of weakly firing neurons. Astrocytes have been shown to increase functional maturation, potentiate the activity of developing neurons and contribute to synapse formation [66-69].
At the neuronal circuitry level, we must bear in mind that endogenous striatal neurons receive excitatory Glu inputs from the cortex and thalamus and DA inputs from the substantia nigra pars compacta. It has been shown that the inhibition of cortico-striatal projections during synaptogenesis alters MSNs maturation by reducing dendritic spine density [67]. The addition of such inputs could have a positive impact on in vitro striatal neuron differentiation and network formation. Thus, the development of a differentiation protocol with integrated cortical and dopaminergic inputs could have a positive impact on in vitro striatal neuron differentiation and network formation.

Based on the relevant gene expression and functional data, in combination with previously published data $[18,19]$, we conclude that the differentiation protocol produces functional neurons with gene expression patterns similar to those observed during functional neuronal maturation in vivo.

\section{Striatal Neuronal Differentiation of hPSC-Derived NPCs Within the Mouse Striatum and Integration into Host Circuitry}

The suitability of this protocol for use as part of cell therapybased treatments was evaluated by the transplantation of hPSC-derived NPCs into the neonatal mouse striatum. In the ideal scenario, which is fully compatible with a cell therapybased approach, transplanted NPCs should differentiate to the desired neuronal type without excessive proliferation. These neurons should then survive for a prolonged period and integrate into the host circuitry.

The transplanted NPCs differentiated to neurons within 1 month of grafting, with no increased number of transplanted cells being observed by 3 months post-transplantation. This demonstrates that the transplanted NPCs integrate local signals to exit the cell cycle and commit to a neuronal identity with a minimal risk of graft overgrowth. This compares favourably to previous work where excessive graft overgrowth was observed following the transplantation of hPSC-derived NPCs into mouse striatum [29, 30]. The majority of the NPC-derived neurons acquired a striatal identity based on the expression of known striatal neuron markers, which correlates well with the in vitro neuronal gene expression data. These neurons also showed higher survival and terminal differentiation in the mouse striatum compared with other studies [68].

A subset of the neurons displayed polarised innervation towards the external globus pallidus (GP) as occurs with endogenous MSNs, indicating that they are integrating into the relevant host circuitry. During development, axon outgrowth and projection are highly regulated by cell-autonomous mechanisms and also by extracellular cues [69, 70]. Several TFs, including CTIP2, ISL1 and EBF1, have been implicated in striatonigral MSN axon outgrowth [37, 71, 72]. Expression 
of these TFs was observed in hPSC-derived neurons both in vitro and post-transplantation, indicating that NPCs are correctly patterned prior to grafting. The combination of NPC intrinsic properties acquired during in vitro differentiation, together with the integration of signals from the host environment, led transplanted cells to behave in a similar manner to host striatal neurons. Furthermore, hNPC-derived neurons established inhibitory synapses (likely GABAergic) and received excitatory synaptic inputs (likely glutamatergic) from host neurons. This mirrors the input-output circuitry of MSNs and suggests that hNPC-derived neurons are also functional within the host circuitry. Remarkably, little evidence of architecturally appropriate neuronal integration, much less circuit reconstruction, has been reported after transplantation of hESC or hiPSC-derived MSNs into the striatum [73]. Based on the behaviour of the transplanted NPCs, we conclude that our differentiation protocol successfully meets all the criteria for use in cell therapy-based strategies.

\section{Conclusion}

Here we perform an in-depth characterisation of an in vitro differentiation protocol that produces functional ventral forebrain neurons, including medium spiny neurons, from hPSCs in just 37 days. We observe that the protocol is robust and reproducible, and that the dynamic changes in gene expression that occur during the protocol mirror those observed during human neurodevelopment. The neurons that are produced in vitro are functionally mature while the protocol is also compatible with cell therapy-based approaches following the successful transplantation of hPSC-derived NPCS which undergo neuronal differentiation and integrate into the host brain circuitry.

Given all of these characteristics, we conclude that this protocol is an ideal platform for the in vitro study of both neurodevelopment and neuronal diseases, including those neurodegenerative disorders that cause alterations during neurogenesis. Due to its robust, reproducible and rapid nature, it is also ideal for use as a drug screening and developmental toxicology platform. Finally, we believe that this protocol has great potential as the basis of cell therapy-based strategies to treat neurodegenerative diseases where there is currently no cure.

Acknowledgements The rat-401 monoclonal anti-nestin antibody developed by S. Hockfield was obtained from the Development Studies Hybridoma Bank developed under the auspices of the NICHD and maintained by the Department of Biological Science, University of Iowa, Iowa City, Iowa.

Funding Information This study was supported by grants from the Ministerio de Ciencia, Innovación y Universidades (Spain), under projects no. SAF2017-84248-P (JCS), SAF 2017-88076-R (JA), FIS201678507-C2-2-P (JS) and RTI2018-099001-B-I00 (JMC); Instituto de Salud Carlos III, Ministerio de Ciencia, Innovación y Universidades and European Regional Development Fund (ERDF) (CIBERNED, to
JA and RETICS (Red de Terapia Celular; RD16/0011/0011 to JCS and RD16/0011/0012 to JMC)), Spain; Generalitat de Catalunya (2017SGR1095 to JA, 2017SGR-1061 to JS and 2017SGR-1408 to JMC), Spain; Comunidad de Madrid (AvanCell-CM; S2017/BMD3692 to JCS), Spain; CHDI foundation (A-14079 to JMC), USA; and ADVANCE(CAT) with the support of ACCIÓ (Catalonia Trade \& Investment; Generalitat de Catalunya) and the European Community under the Catalonian ERDF operational program 2014-2020, Spain. This research is part of MESOBRAIN. The MESO-BRAIN Project has received funding from the European Union's Horizon 2020 Research and Innovation Programme under grant agreement no. 713140 .

Open Access This article is licensed under a Creative Commons Attribution 4.0 International License, which permits use, sharing, adaptation, distribution and reproduction in any medium or format, as long as you give appropriate credit to the original author(s) and the source, provide a link to the Creative Commons licence, and indicate if changes were made. The images or other third party material in this article are included in the article's Creative Commons licence, unless indicated otherwise in a credit line to the material. If material is not included in the article's Creative Commons licence and your intended use is not permitted by statutory regulation or exceeds the permitted use, you will need to obtain permission directly from the copyright holder. To view a copy of this licence, visit http://creativecommons.org/licenses/by/4.0/.

\section{References}

1. Urb $\tilde{A}_{i n}$ N, Guillemot F (2014) Neurogenesis in the embryonic and adult brain: same regulators, different roles. Front Cell Neurosci 8: 396. https://doi.org/10.3389/fncel.2014.00396

2. Wiatr K, Szlachcic WJ, Trzeciak M, Figlerowicz M, Figiel M (2018) Huntington disease as a neurodevelopmental disorder and early signs of the disease in stem cells. Mol Neurobiol 55:33513371. https://doi.org/10.1007/s12035-017-0477-7

3. Molero AE, Gokhan S, Gonzalez S, Feig JL, Alexandre LC, Mehler MF (2009) Impairment of developmental stem cell-mediated striatal neurogenesis and pluripotency genes in a knock-in model of Huntington's disease. Proc Natl Acad Sci U S A 106:21900 21905. https://doi.org/10.1073/pnas.0912171106

4. HD iPSC Consortium (2017) Developmental alterations in Huntington's disease neural cells and pharmacological rescue in cells and mice. Nat Neurosci 20:648-660. https://doi.org/10.1038/ nn. 4532

5. Mahla RS (2016) Stem cells applications in regenerative medicine and disease therapeutics. Int J Cell Biol 2016:6940283. https://doi. org $/ 10.1155 / 2016 / 6940283$

6. Ma W, Tavakoli T, Derby E et al (2008) Cell-extracellular matrix interactions regulate neural differentiation of human embryonic stem cells. BMC Dev Biol 8:1-13. https://doi.org/10.1186/1471213X-8-90

7. Smith LR, Cho S, Discher DE (2018) Stem cell differentiation is regulated by extracellular matrix mechanics. Physiology (Bethesda) 33:16-25. https://doi.org/10.1152/physiol.00026.2017

8. Fjodorova M, Noakes Z, Li M (2015) How to make striatal projection neurons. Neurogenesis 2:e1100227. https://doi.org/10.1080/ 23262133.2015.1100227

9. Hemmati-Brivanlou A, Melton D (1997) Vertebrate neural induction. Annu Rev Neurosci 20:43-60. https://doi.org/10.1146/ annurev.neuro.20.1.43

10. Chambers SM, Fasano CA, Papapetrou EP et al (2009) Highly efficient neural conversion of human ES and iPS cells by dual 
inhibition of SMAD signaling. Nat Biotechnol 27:275-280. https:// doi.org/10.1038/nbt.1529

11. Heldin CH, Miyazono K, ten Dijke P (1997) TGF-beta signalling from cell membrane to nucleus through SMAD proteins. Nature 390:465-471. https://doi.org/10.1038/37284

12. Bengoa-Vergniory N, Kypta RM (2015) Canonical and noncanonical Wnt signaling in neural stem/progenitor cells. Cell Mol Life Sci 72:4157-4172. https://doi.org/10.1007/s00018-015-2028-6

13. Monuki ES, Porter FD, Walsh CA (2001) Patterning of the dorsal telencephalon and cerebral cortex by a roof plate-Lhx2 pathway. Neuron 32:591-604. https://doi.org/10.1016/S0896-6273(01) 00504-9

14. Ulloa F, Martí E (2010) Wnt won the war: antagonistic role of Wnt over Shh controls dorso-ventral patterning of the vertebrate neural tube. Dev Dyn 239:69-76. https://doi.org/10.1002/dvdy.22058

15. Danjo T, Eiraku M, Muguruma K, Watanabe K, Kawada M, Yanagawa Y, Rubenstein JL, Sasai Y (2011) Subregional specification of embryonic stem cell-derived ventral telencephalic tissues by timed and combinatory treatment with extrinsic signals. $\mathrm{J}$ Neurosci 31:1919-1933. https://doi.org/10.1523/JNEUROSCI. 5128-10.2011

16. Weick JP (2016) Functional properties of human stem cell-derived neurons in health and disease. Stem Cells Int 2016:4190438. https:// doi.org/10.1155/2016/4190438

17. Edlund T, Jessell TM (1999) Progression from extrinsic to intrinsic signaling in cell fate specification: a view from the nervous system. Cell 96:211-224

18. Telezhkin V, Schnell C, Yarova P, Yung S, Cope E, Hughes A, Thompson BA, Sanders P et al (2016) Forced cell cycle exit and modulation of GABA A, CREB, and GSK $3 \beta$ signaling promote functional maturation of induced pluripotent stem cell-derived neurons. Am J Physiol - Cell Physiol 310:C520-C541. https://doi.org/ 10.1152/ajpcell.00166.2015

19. Straccia M, Garcia-Diaz Barriga G, Sanders P, Bombau G, Carrere J, Mairal PB, Vinh NN, Yung S et al (2015) Quantitative highthroughput gene expression profiling of human striatal development to screen stem cell-derived medium spiny neurons. Mol Ther Methods Clin Dev 2:15030. https://doi.org/10.1038/mtm. 2015.30

20. Orlandi JG, Fernández-García S, Comella-Bolla A, et al (2017) NETCAL: an interactive platform for large-scale, NETwork and population dynamics analysis of CALcium imaging recordings https://doi.org/10.5281/ZENODO.1119026

21. Freund Y (2009) A more robust boosting algorithm. arXiv Prepr arXiv09052138

22. Nakai J, Ohkura M, Imoto K (2001) A high signal-to-noise $\mathrm{Ca}(2+)$ probe composed of a single green fluorescent protein. Nat Biotechnol 19:137-141. https://doi.org/10.1038/84397

23. Lütcke H, Gerhard F, Zenke F, Gerstner W, Helmchen F (2013) Inference of neuronal network spike dynamics and topology from calcium imaging data. Front Neural Circuits 7:201. https://doi.org/ 10.3389/fncir.2013.00201

24. Abdi H, Williams LJ (2010) Principal component analysis. Wiley Interdiscip Rev Comput Stat 2:433-459. https://doi.org/10.1002/ wics. 101

25. Ozden I, Lee HM, Sullivan MR, et al (2008) Identification and clustering of event patterns from in vivo multiphoton optical recordings of neuronal ensembles. 495-503. https://doi.org/10.1152/jn. 01310.2007 .

26. (2018) Guidance Document on Good In Vitro Method Practices (GIVIMP). OECD

27. Englund C, Fink A, Charmaine L et al (2005) Pax6, Tbr2, and Tbr1 are expressed sequentially by radial glia, intermediate progenitor cells, and postmitotic neurons in developing neocortex. J Neurosci 25:247-251. https://doi.org/10.1523/JNEUROSCI.289904.2005
28. Straccia M, Carrere J, Rosser AE, Canals JM (2016) Human tDARPP is induced during striatal development. Neuroscience 333:320-330. https://doi.org/10.1016/j.neuroscience.2016.07.022

29. Delli Carri A, Onorati M, Castiglioni V, Faedo A, Camnasio S, Toselli M, Biella G, Cattaneo E (2013) Human pluripotent stem cell differentiation into authentic striatal projection neurons. Stem Cell Rev Reports 9:461-474. https://doi.org/10.1007/s12015-0139441-8

30. Aubry L, Bugi A, Lefort N, Rousseau F, Peschanski M, Perrier AL (2008) Striatal progenitors derived from human ES cells mature into DARPP32 neurons in vitro and in quinolinic acid-lesioned rats. Proc Natl Acad Sci U S A 105:16707-16712. https://oi.org/10. 1073/pnas.0808488105

31. Campbell K (2003) Dorsal-ventral patterning in the mammalian telencephalon. Curr Opin Neurobiol 13:50-56. https://doi.org/10. 1016/S0959-4388(03)00009-6

32. Evans AE, Kelly CM, Precious VS, Rosser AE (2012) Molecular regulation of striatal development: a review. Anat Res Int 2012:114. https://doi.org/10.1155/2012/106529

33. Li X-J, Zhang X, Johnson MA, Wang ZB, Lavaute T, Zhang SC (2009) Coordination of sonic hedgehog and Wnt signaling determines ventral and dorsal telencephalic neuron types from human embryonic stem cells. Development 136:4055-4063. https://doi. org/10.1242/dev.036624

34. Liu Y, Weick JP, Liu H, Krencik R, Zhang X, Ma L, Zhou GM, Ayala $\mathrm{M}$ et al (2013) Medial ganglionic eminence-like cells derived from human embryonic stem cells correct learning and memory deficits. Nat Biotechnol 31:440-447. https://doi.org/10.1038/nbt. 2565

35. Arber C, Precious VS, Cambray S et al (2015) Activin A directs striatal projection neuron differentiation of human pluripotent stem cells. Development 142:1375-1386. https://doi.org/10.1242/dev. 117093

36. Molyneaux BJ, Arlotta P, Menezes JRL, Macklis JD (2007) Neuronal subtype specification in the cerebral cortex. Nat Rev Neurosci 8:427-437. https://doi.org/10.1038/nrn2151

37. Lobo MK, Yeh C, Yang XW (2008) Pivotal role of early B-cell factor 1 in development of striatonigral medium spiny neurons in the matrix compartment. J Neurosci Res 86:2134-2146. https://doi. org/10.1002/jnr.21666

38. Martín-Ibáñez R, Crespo E, Esgleas M et al (2012) Helios transcription factor expression depends on Gsx2 and Dlx1\&amp;2 function in developing striatal matrix neurons. Stem Cells Dev 21. https:// doi.org/10.1089/scd.2011.0607

39. Long JE, Swan C, Liang WS et al (2009) Dlx1\&2 and Mash1 transcription factors control striatal patterning and differentiation through parallel and overlapping pathways. J Comp Neurol 512: 556-572. https://doi.org/10.1002/cne.21854

40. Garcia-Dominguez M, Poquet C, Garel S, Charnay P (2003) Ebf gene function is required for coupling neuronal differentiation and cell cycle exit. Development 130:6013-6025. https://doi.org/10. 1242/dev.00840

41. Lobo MK, Karsten SL, Gray M, Geschwind DH, Yang XW (2006) FACS-array profiling of striatal projection neuron subtypes in juvenile and adult mouse brains. Nat Neurosci 9:443-452. https://doi. org/10.1038/nn1654

42. Eisenstat DD, Liu JK, Mione M, Zhong W, Yu G, Anderson SA, Ghattas I, Puelles L et al (1999) DLX-1, DLX-2, and DLX-5 expression define distinct stages of basal forebrain differentiation. J Comp Neurol 414:217-237

43. Lin L, Yuan J, Sander B, Golas MM (2015) In vitro differentiation of human neural progenitor cells into striatal GABAergic neurons. Stem Cells Transl Med 4:775-788. https://doi.org/10.5966/sctm. 2014-0083

44. Arlotta P, Molyneaux BJ, Jabaudon D, Yoshida Y, Macklis JD (2008) Ctip2 controls the differentiation of medium spiny neurons 
and the establishment of the cellular architecture of the striatum. J Neurosci 28:622-632. https://doi.org/10.1523/JNEUROSCI.298607.2008

45. Gaspard N, Bouschet T, Hourez R, Dimidschstein J, Naeije G, van den Ameele J, Espuny-Camacho I, Herpoel A et al (2008) An intrinsic mechanism of corticogenesis from embryonic stem cells. Nature 455:351-357. https://doi.org/10.1038/nature07287

46. Cánovas J, Berndt FA, Sepúlveda H, Aguilar R, Veloso FA, Montecino M, Oliva C, Maass JC et al (2015) The specification of cortical subcerebral projection neurons depends on the direct repression of TBR1 by CTIP1/BCL11a. J Neurosci 35:75527564. https://doi.org/10.1523/JNEUROSCI.0169-15.2015

47. Simon HH, Bhatt L, Gherbassi D et al (2003) Midbrain dopaminergic neurons: determination of their developmental fate by transcription factors. Ann N Y Acad Sci 991:36-47

48. Arenas E, Denham M, Villaescusa JC (2015) How to make a midbrain dopaminergic neuron. Development 142:1918-1936. https:// doi.org/10.1242/dev.097394

49. Martínez-Cerdeño V, Noctor SC, Espinosa A, Ariza J, Parker P, Orasji S, Daadi MM, Bankiewicz K et al (2010) Embryonic MGE precursor cells grafted into adult rat striatum integrate and ameliorate motor symptoms in 6-OHDA-Lesioned rats. Cell Stem Cell 6: 238-250. https://doi.org/10.1016/j.stem.2010.01.004

50. Marin O, Anderson SA, Rubenstein JL (2000) Origin and molecular specification of striatal interneurons. J Neurosci 20:6063-6076

51. Cobos I, Calcagnotto ME, Vilaythong AJ, Thwin MT, Noebels JL, Baraban SC, Rubenstein JL (2005) Mice lacking Dlx1 show subtype-specific loss of interneurons, reduced inhibition and epilepsy. Nat Neurosci 8:1059-1068. https://doi.org/10.1038/nn1499

52. Tepper JM, Koós T, Ibanez-Sandoval O, Tecuapetla F, Faust TW, Assous M (2018) Heterogeneity and diversity of striatal GABAergic interneurons: update 2018. Front Neuroanat 12:91. https://doi.org/10.3389/fnana.2018.00091

53. Song M, Mohamad O, Chen D, Yu SP (2013) Coordinated development of voltage-gated $\mathrm{Na}+$ and $\mathrm{K}+$ currents regulates functional maturation of forebrain neurons derived from human induced pluripotent stem cells. Stem Cells Dev 22:1551-1563. https://doi.org/ $10.1089 / \mathrm{scd} .2012 .0556$

54. Ghosh A, Greenberg M (1995) Calcium signaling in neurons: molecular mechanisms and cellular consequences. Science (80- ) 268: 239-247. https://doi.org/10.1126/science.7716515

55. Greer PL, Greenberg ME (2008) From synapse to nucleus: calciumdependent gene transcription in the control of synapse development and function. Neuron 59:846-860. https://doi.org/10.1016/j. neuron.2008.09.002

56. Lu WY, Man HY, Ju W, Trimble WS, MacDonald J, Wang YT (2001) Activation of synaptic NMDA receptors induces membrane insertion of new AMPA receptors and LTP in cultured hippocampal neurons. Neuron 29:243-254. https://doi.org/10.1016/S08966273(01)00194-5

57. Watanabe H, Yamashita T, Saitoh N, Kiyonaka S, Iwamatsu A, Campbell KP, Mori Y, Takahashi T (2010) Involvement of Ca2+ channel synprint site in synaptic vesicle endocytosis. J Neurosci 30: 655-660. https://doi.org/10.1523/JNEUROSCI.3214-09.2010

58. Sheng Z-H, Rettig J, Cook T, Catterall WA (1996) Calciumdependent interaction of $\mathrm{N}$-type calcium channels with the synaptic core complex. Nature 379:451

59. Heidelberger R, Heinemann C, Neher E, Matthews G (1994) Calcium dependence of the rate of exocytosis in a synaptic terminal. Nature 371:513
60. Xu X, Lee YJ, Holm JB et al (2011) The Ca2+ channel $\beta 4 \mathrm{c}$ subunit interacts with heterochromatin protein 1 via a PXVXL binding motif. J Biol Chem 286:9677-9687. https://doi.org/10.1074/jbc. M110.187864

61. Hibino H, Pironkova R, Onwumere O, Rousset M, Charnet P, Hudspeth AJ, Lesage F (2003) Direct interaction with a nuclear protein and regulation of gene silencing by a variant of the $\mathrm{Ca} 2+$ -channel beta 4 subunit. Proc Natl Acad Sci U S A 100:307-312. https://doi.org/10.1073/pnas.0136791100

62. Furukawa M, Tsukahara T, Tomita K, Iwai H, Sonomura T, Miyawaki S, Sato T (2017) Neonatal maternal separation delays the GABA excitatory-to-inhibitory functional switch by inhibiting KCC2 expression. Biochem Biophys Res Commun 493:12431249. https://doi.org/10.1016/j.bbrc.2017.09.143

63. Olde Engberink AHO, Meijer JH, Michel S (2018) Chloride cotransporter $\mathrm{KCC} 2$ is essential for GABAergic inhibition in the SCN. Neuropharmacology 138:80-86. https://doi.org/10.1016/j. neuropharm.2018.05.023

64. Watanabe M, Fukuda A (2015) Development and regulation of chloride homeostasis in the central nervous system. Front Cell Neurosci 9:371. https://doi.org/10.3389/fncel.2015.00371

65. Grewe BF, Langer D, Kasper H, Kampa BM, Helmchen F (2010) High-speed in vivo calcium imaging reveals neuronal network activity with near-millisecond precision. Nat Methods 7:399-405. https://doi.org/10.1038/nmeth.1453

66. Baldwin KT, Eroglu C (2017) Molecular mechanisms of astrocyteinduced synaptogenesis. Curr Opin Neurobiol 45:113-120. https:// doi.org/10.1016/j.conb.2017.05.006

67. Kozorovitskiy Y, Saunders A, Johnson CA et al (2012) Recurrent network activity drives striatal synaptogenesis. Nature 489:326. https://doi.org/10.1038/nature11372

68. Golas MM, Sander B (2016) Use of human stem cells in Huntington disease modeling and translational research. Exp Neurol 278:76-90. https://doi.org/10.1016/j.expneurol.2016.01. 021

69. Chauvet S, Cohen S, Yoshida Y, Fekrane L, Livet J, Gayet O, Segu L, Buhot MC et al (2007) Gating of Sema3E/PlexinD1 signaling by neuropilin-1 switches axonal repulsion to attraction during brain development. Neuron 56:807-822

70. Uemura M, Nakao S, Suzuki ST et al (2007) OL-Protocadherin is essential for growth of striatal axons and thalamocortical projections. Nat Neurosci 10:1151

71. Ehrman LA, Mu X, Waclaw RR, Yoshida Y, Vorhees CV, Klein WH, Campbell K (2013) The LIM homeobox gene Isl1 is required for the correct development of the striatonigral pathway in the mouse. Proc Natl Acad Sci U S A 110:E4026-E4035. https://doi. org/10.1073/pnas.1308275110

72. Lu K-M, Evans SM, Hirano S, Liu F-C (2014) Dual role for Islet-1 in promoting striatonigral and repressing striatopallidal genetic programs to specify striatonigral cell identity. Proc Natl Acad Sci 111: E168-E177. https://doi.org/10.1073/pnas.1319138111

73. Goldman SA (2016) Stem and progenitor cell-based therapy of the central nervous system: hopes, hype, and wishful thinking. Cell Stem Cell 18:174-188. https://doi.org/10.1016/j.stem.2016.01.012

Publisher's Note Springer Nature remains neutral with regard to jurisdictional claims in published maps and institutional affiliations. 


\section{Affiliations}

\section{Andrea Comella-Bolla ${ }^{1,2,3,4,5}$ (D) Javier G. Orlandi ${ }^{6,7,8}$ (D) $\cdot$ Andrés Miguez ${ }^{1,2,3,4,5}$ (D) Marco Straccia $^{1,2,3,4,5}$ (ID) María García-Bravo ${ }^{9,10}$ (D) - Georgina Bombau ${ }^{1,2,3,4,5}$ • Mireia Galofré ${ }^{1,2,3,4,5}$ (D) Phil Sanders ${ }^{1,2,3,4,5}$ (D) Jordi Carrere $^{1,2,3,4,5}$ (D) José Carlos Segovia ${ }^{9,10}$ (D) Joan Blasi ${ }^{11}$ (D) Nicholas D. Allen $^{12}$. Jordi Alberch $^{2,3,4,5,6}$ (D) Jordi Soriano $^{7,13}$ (D) - Josep M. Canals ${ }^{1,2,3,4,5}$ (ID)}

1 Laboratory of Stem Cells and Regenerative Medicine, Department of Biomedicine, Faculty of Medicine and Health Science, University of Barcelona, Barcelona, Spain

2 Production and validation center of advanced therapies (Creatio), Faculty of Medicine and Health Science, University of Barcelona, Barcelona, Spain

3 Institute of Neurosciences, University of Barcelona, Barcelona, Spain

4 Networked Biomedical Research Centre for Neurodegenerative Disorders, Barcelona, Spain

5 August Pi i Sunyer Biomedical Research Institute (IDIBAPS), Barcelona, Spain

6 Pathophysiology of Neurodegenerative Disease. Laboratory, Department of Biomedicine, Faculty of Medicine and Health Science, University of Barcelona, Barcelona, Spain

7 Departament de Física de la Matèria Condensada, University of Barcelona, Barcelona, Spain
8 Department of Physics and Astronomy, University of Calgary, Calgary, Canada

9 Differentiation and Cytometry Unit, Division of Hematopoietic Innovative Therapies, Centro de Investigaciones Energéticas Medioambientales y Tecnológicas and Centro de Investigación Biomédica en Red de Enfermedades Raras (CIEMAT/CIBERER), Madrid, Spain

10 Advanced Therapies Unit, Instituto de Investigación Sanitaria Fundación Jiménez Díaz (IIS-FJD, UAM), 28040 Madrid, Spain

11 Laboratory of Cellular and Molecular Neurobiology, Department Pathology and Experimental Therapeutics, Faculty of Medicine and Health Science, Biomedical Research Institute of Bellvitge (IDIBELL), University of Barcelona, Barcelona, Spain

12 Cardiff Repair Group, School of Biosciences and medicine, Cardiff University, Cardiff, Wales, UK

13 Institute of Complex Systems (UBICS), University of Barcelona, Barcelona, Spain 